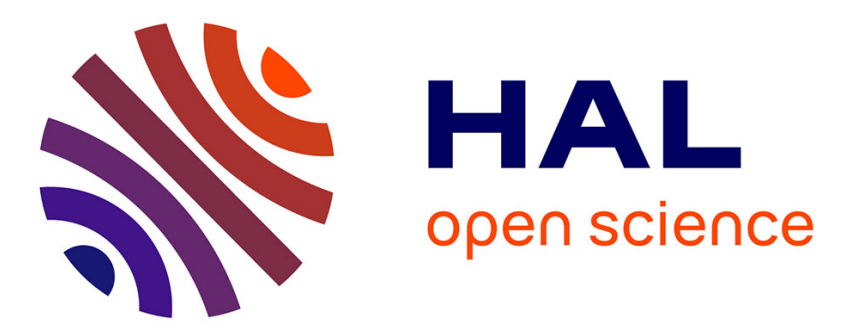

\title{
Un centre domanial dans la vallée de la Tave: la villa de Mayran (Saint-Victor-la-Coste, Gard)
}

\author{
Loïc Buffat, Hervé Petitot, Laurent Vidal, Josselyne Guerre, Aurélie
}

Masbernat-Buffat, Elie Pélaquier

\section{- To cite this version:}

Loïc Buffat, Hervé Petitot, Laurent Vidal, Josselyne Guerre, Aurélie Masbernat-Buffat, et al.. Un centre domanial dans la vallée de la Tave: la villa de Mayran (Saint-Victor-la-Coste, Gard). Revue archéologique de Narbonnaise, 2006, 38-39, pp.225-282. hal-02267017

\section{HAL Id: hal-02267017 \\ https://hal.science/hal-02267017}

Submitted on 18 Aug 2019

HAL is a multi-disciplinary open access archive for the deposit and dissemination of scientific research documents, whether they are published or not. The documents may come from teaching and research institutions in France or abroad, or from public or private research centers.
L'archive ouverte pluridisciplinaire HAL, est destinée au dépôt et à la diffusion de documents scientifiques de niveau recherche, publiés ou non, émanant des établissements d'enseignement et de recherche français ou étrangers, des laboratoires publics ou privés. 


\title{
Un centre domanial dans la vallée de la Tave : la villa de Mayran (Saint-Victor-la-Coste, Gard)
}

\author{
Loïc BUFFAT, Hervé PETITOT, Laurent VIDAL \\ avec la collaboration de Josselyne GUERRE, Aurélie MASBERNAT et Élie PÉLAQUIER
}

En hommage à notre ami Louis Faraud (1938-2005)

\begin{abstract}
Résumé : L'archéologie de la villa connaît depuis une décennie un regain d'intérêt dans le département du Gard. La publication des fouilles et prospections réalisées à Mayran (Saint-Victor-la-Coste) tente de rendre compte du dynamisme de cette recherche thématique. Dans ce cas, un des aspects les plus intéressants est certainement de pouvoir suivre l'histoire du site sur la longue durée, du I ${ }^{\text {er }}$ s. av. J.-C. jusqu'à nos jours. A l'époque romaine, la villa se présente comme un ensemble important, qui sera doté, au Bas-Empire, d'installations vinicoles et d'un aqueduc à la morphologie peu commune. La villa sera occupée tout au long du haut Moyen Âge (VI ${ }^{\mathrm{e}}-\mathrm{X}^{\mathrm{e}} \mathrm{s}$.), puis au Moyen Âge médian (XI'-XIII ${ }^{\mathrm{e}} \mathrm{s}$.). Mayran illustre ainsi une caractéristique importante des villae de la région : leur rôle durable dans l'organisation des campagnes.

Mots-clés : Languedoc-Roussillon, époque romaine, haut Moyen Âge, villa, viticulture, hydraulique, métallurgie.
\end{abstract}

Abstract: Since the last decade, archaeology of the villa has a renewal of interest in the French department of Gard. The publication of the excavations and prospections realized on the site of Mayran (St Victor La Coste) reflects the dynamism of this research. One of the most interesting results is the historical reconstruction of the site on a long period of time, i.e. from the $\mathrm{I}^{\text {st }}$ century $\mathrm{BC}$ up to present time. During the Roman period, the villa was a large complex including, in the Late Empire, a wine-making equipment and an acqueduct with an uncommon morphology. The period of occupation extends from Early Middle Age (VI ${ }^{\text {th }}-\mathrm{X}^{\text {th }}$ century) up to the High Middle Age (XI ${ }^{\text {th }}$ XIII ${ }^{\text {th }}$ century). The Mayran villa gives us an example of an important characteristic of the villae in the Gard region : their lasting role in the organization of the countryside.

Keywords : Languedoc, Roman period, early Middle Age, villa, wine-making, hydraulic, metallurgy.

\section{PRÉSENTATION}

Situé dans la vallée de la Tave, au nord-est du département du Gard, le site de Mayran, à Saint-Victor-la-Coste (fig. 1), constitue l'un des établissements antiques les plus étendus de cette région. Il présente une occupation continue depuis le Ir s. av. J.-C. jusqu'à nos jours. Nous y avons conduit une fouille de sauvetage entre 1995 et 1998 , ainsi que des prospections méthodiques en 2002. Les résultats de ces travaux proposent de restituer un grand centre domanial antique, ayant produit du vin au BasEmpire. L'établissement est équipé à la même période d'un important aqueduc souterrain, sur lequel les fouilles ont apporté quelques précisions. L'étude de ces structures permettra d'envisager les modalités d'évolution d'un établissement de première importance dans l'histoire locale.
Par ailleurs, nos fouilles ont permis d'explorer de nombreux silos médiévaux se développant à l'emplacement de l'établissement romain. Leur étude permettra d'entrevoir les évolutions du site durant le haut Moyen Âge.

L'étude du site s'intègre dans un programme plus large, portant sur l'occupation du sol dans la vallée de la Tave. Entamée au début des années 1990, cette recherche collective s'est développée selon des axes somme toute classiques. Elle a consisté dans un premier temps en des prospections extensives, conduites entre 1991 et 1995 (H. Petitot dir.), qui ont permis de repérer près de 300 sites archéologiques, dont 165 sites gallo-romains. Des résultats préliminaires de ces recherches ont été présentés au fil des années dans différentes contributions (Buffat 1994, 1996; CAG 30/2, 30/3; Buffat et al. 2003; Buffat 2004). 


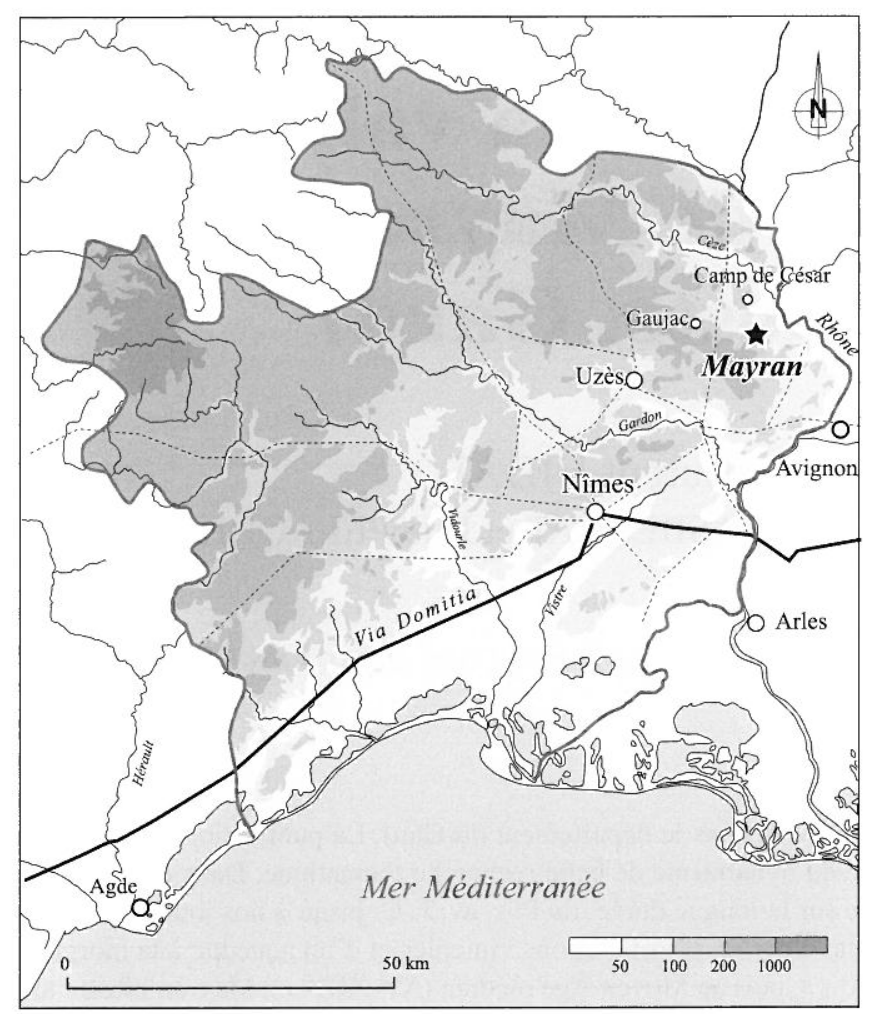

Fig. 1. Localisation du site de Mayran.

Dans un second temps, nos efforts se sont concentrés sur la fouille d'établissements gallo-romains. Ces fouilles ont porté sur des sites de taille et d'importance diverses. Il fallait effectivement envisager les différentes catégories de l'habitat, pour réfléchir sur l'organisation du peuplement au sein de notre micro-région. C'est ce qui a motivé la fouille du petit établissement de la Roquette à Cavillargues (Robin-Petitot, Petitot in BSR LR 2000, 7172), du site annexe d'Aubarnes à Tresques (Buffat, Petitot 1996) et plus récemment du site des Aumignanes (ferme? petite villa?) à Saint-Victor-la-Coste (fouille S. Alix). Dans ce programme de fouille, l'exploration des villae a occupé une place particulièrement importante. Il faut dire qu'à l'origine de nos travaux, cette catégorie d'habitat restait fort mal documentée. Il y avait donc ici un large champ d'étude, qui fut par la suite investi par l'un de nous dans le cadre d'une thèse de III' cycle (Buffat 2004). Depuis, la connaissance des villae a enregistré des progrès significatifs, grâce à plusieurs fouilles. Ainsi l'exploration intégrale du site de la Ramière (Roquemaure), situé sur le tracé du TGV Méditerranée, a apporté des informations tout à fait déterminantes (Barberan et al. 2002). La fouille, plus ponctuelle, de la villa des Charboniers (SaintPaul-les-Fonts) est venue compléter ce tableau (Esteban et al. 2000). L'exploration partielle de la villa de Mayran, dont les résultats sont présentés ici, a également apporté son lot d'informations.

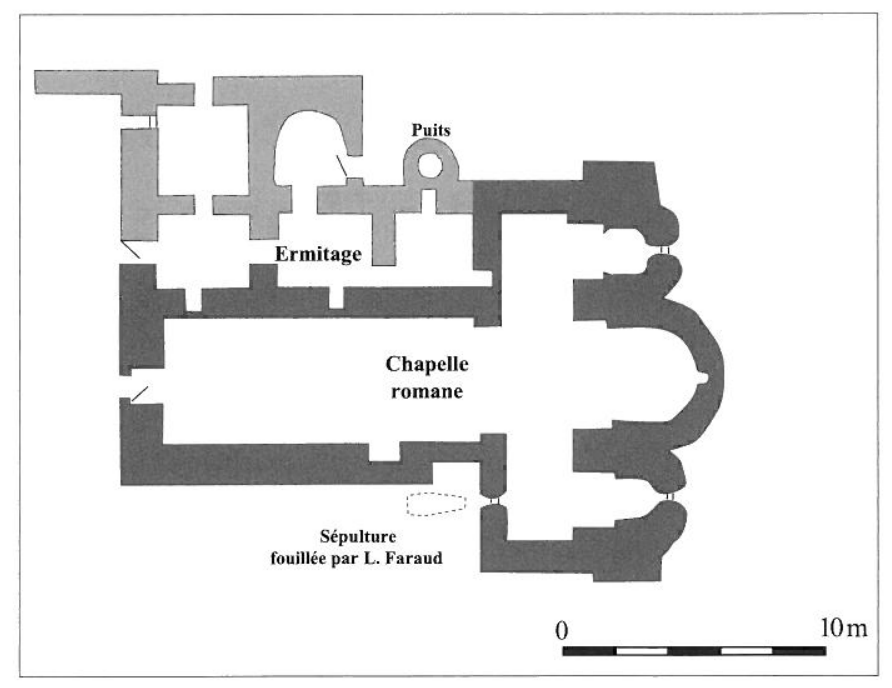

Fig. 2. Plan de la chapelle et de l'ermitage de Mayran (DAO J. Guerre, d'après un relevé de L. Faraud).

\subsection{Historique des recherches}

La fouille réalisée à Mayran fait suite à une longue série d'observations archéologiques. La famille Faraud, propriétaire d'une partie des terres où s'étendent les vestiges, a mené, depuis trois générations, une surveillance régulière du gisement. Ainsi, dès 1952, M. Faraud signalait, dans une série de petits articles, l'abondance des fragments de poteries et des monnaies gallo-romaines à l'est de la chapelle Notre-Dame de Mayran. Il soulignait déjà l'importante occupation que le site avait dû connaître durant l'Antiquité, en s'appuyant notamment sur la découverte, par son père, d'un puits (Faraud 1952, 4). En 1992, un nettoyage de ce puits révéla l'existence d'une canalisation souterraine. De 1970 à 1980, C. Belin recueillit plus de 300 monnaies lors de prospections magnétiques, pour l'essentiel des petits bronzes du Bas-Empire. La découverte d'émissions médiévales (mérovingiennes, carolingiennes et féodales) posait la question d'une occupation durant le haut Moyen Âge, que laissait du reste présager la proximité d'une belle chapelle romane. L'hypothèse fut confirmée, à plusieurs reprises, par la découverte de tombes au sud de l'édifice. En 1971, L. Faraud dégagea, sous un arc de décharge de la chapelle, une sépulture médiévale sous lauzes à laquelle était associée une cruche en céramique kaolinitique réductrice (fig. 2; Faraud 1972). En 1975, ce sont trois niveaux superposés d'inhumations qui furent observés à l'intérieur du parc de la chapelle, lors de l'installation d'un câble électrique souterrain (information L. Faraud). Enfin, plus au sud, en préalable à la construction de l'un des murs de clôture du parc, une fouille de sauvetage, menée par D. Paya en 1993, dégagea deux tombes (une d'adulte et une d'enfant). En 1992, D. Goury effectua une prospec- 
tion intégrale du gisement, où il estima l'étendue des artefacts à $30000 \mathrm{~m}^{2}$ (Goury, Leclaire 1992, 21). De nouveaux repérages menés en 1993 par H. Petitot et le groupe archéologique de la vallée de la Tave corrigèrent cette première estimation à la hausse $\left(40000 \mathrm{~m}^{2}\right)$. Quelques traces d'une occupation durant le Néolithique ou l'âge du Bronze furent également reconnues.

Ces différentes découvertes permettaient d'envisager une occupation importante du site, durant l'époque romaine (Haut et Bas-Empire) et le Moyen Âge. La large superficie du site posait le problème de son statut. S'agissait-il d'une grande villa ou d'une agglomération? Nos travaux penchent vivement pour l'hypothèse d'une villa, comme nous l'exposerons dans les pages qui suivent.

\subsection{Le cadre géographique}

Le gisement de Mayran est implanté à environ $2 \mathrm{~km}$ au nord-est de 1'actuel village de Saint-Victor-la-Coste (fig. 4), et à $1 \mathrm{~km}$ du hameau de Palus. Situé sur un coteau, à une altitude moyenne de $100 \mathrm{~m}$, il est installé sur différents types de sols: à l'ouest sur des terrains de cailloutis würmiens, à l'est sur des sols sablonneux du pliocène supérieur. Si l'on en croit les agronomes, les sols de cailloutis würmiens sont peu favorables pour l'agriculteur, sauf pour la viticulture moderne, alors que les sables présentent des capacités agrologiques satisfaisantes. Les cultures actuelles sont très largement dominées par la vigne, qui n'est concurrencée que par quelques rares arbres fruitiers. Au sud, le site profite de la proximité d'un petit cours d'eau saisonnier, le ruisseau de Cubelle, qui prend sa source au pied des falaises barremiennes de Saint-Victor. À sec le plus clair de l'année, il constitue plus un "vallat" qu'un véritable ruisseau. Il s'insinue entre deux collines, Serre Biau, petite éminence de tufs quaternaires, et Mont $\mathrm{Cau}$, terrasse würmienne, occupée il y encore peu, par la garrigue. Le gisement bénéficie, à l'instar de nombreuses villae, d'une situation intermédiaire, à l'interface de la plaine alluviale et des coteaux boisés. Les ressources du coteau (bois, pierre, pâtures) apportent ici un complément précieux à celles de la plaine, dévolue à l'agriculture.

\subsection{Sources écrites}

\subsubsection{Les stèles}

Trois stèles funéraires sont remployées dans la chapelle ou ont été retrouvées à proximité. Il n'est pas certain qu'elles proviennent du site, les contextes de découverte n'étant pas précis. La première (CIL, XII, 2793), prise dans un des piliers de la chapelle, porte l'inscription:

\section{D.M \\ Q FRONTONI \\ QVINTINI \\ Q.F. PRIMVLVS \\ ET.F.SEVERUS \\ ET.F QVINTILLA \\ PATR OPTIMO}

«Aux dieux Mânes de Quintus Frontonius Quintini; Quintus Frontonius Primulus, Frontonius Severus, Frontonius Quintilla à leur excellent père».

M. Christol, qui s'est intéressé à la répartition du gentilice Frontonius (fig. 3), note qu'il est surtout représenté dans la cité de Nîmes (Buffat et al. 2002). On notera d'ailleurs qu'un Frontonius est connu sur la commune proche de Tresques (CIL, XII, 2767).

La seconde stèle porte l'épitaphe d'un magistrat municipal (CIL, XII, 2794, révisée par M. Christol in CAG $30 / 3$, p. $\left.661, \mathrm{n}^{\circ} 302,9\right)$ :

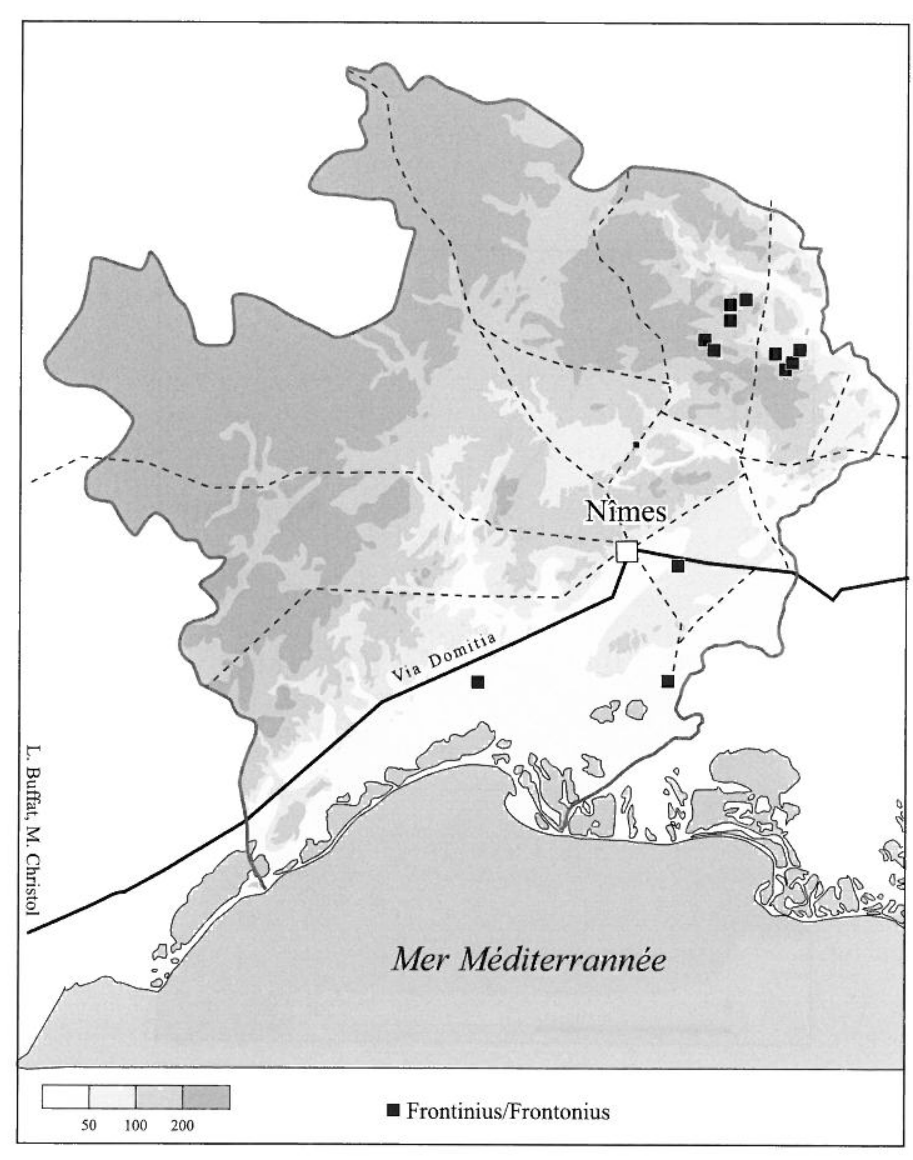

Fig. 3. Répartition du gentilice Frontinius / Frontonius dans la Cité de Nîmes (d'après M. Christol). On constate que ce gentilice est bien représenté dans la vallée de la Tave. 


\section{D.M. \\ M. TVLLI PAVLLINI \\ IIII VIR. AB. AER \\ PONTIF \\ M. TVLLIVS AMMIAN \\ PATRI PIISSIMO}

«Aux dieux Mânes de Marcus Tullius Paullinus, quattuorvir trésorier, pontife; Marcus Tullius Ammianius à son excellent père».

Longtemps restée à l'intérieur de la chapelle, elle a été déplacée et demeure actuellement introuvable. Cette stèle est très intéressante car elle mentionne un personnage investi de hautes responsabilités dans le cadre régional.

La troisième stèle se situe actuellement dans un petit oratoire à l'entrée du parc de la chapelle. Très fragmentaire, elle a été retrouvée dans le puits accolé à l'ermitage de Mayran (CAG 30/3, p. 661, n 302,9$)$. Elle porte l'inscription:

\section{D}

PRI...

Le D situé sur la première ligne pourrait être le reste d'une évocation aux dieux mânes. PRI pourrait se rattacher à Primula ou Primulus, gentilice bien représenté dans la cité de Nîmes.

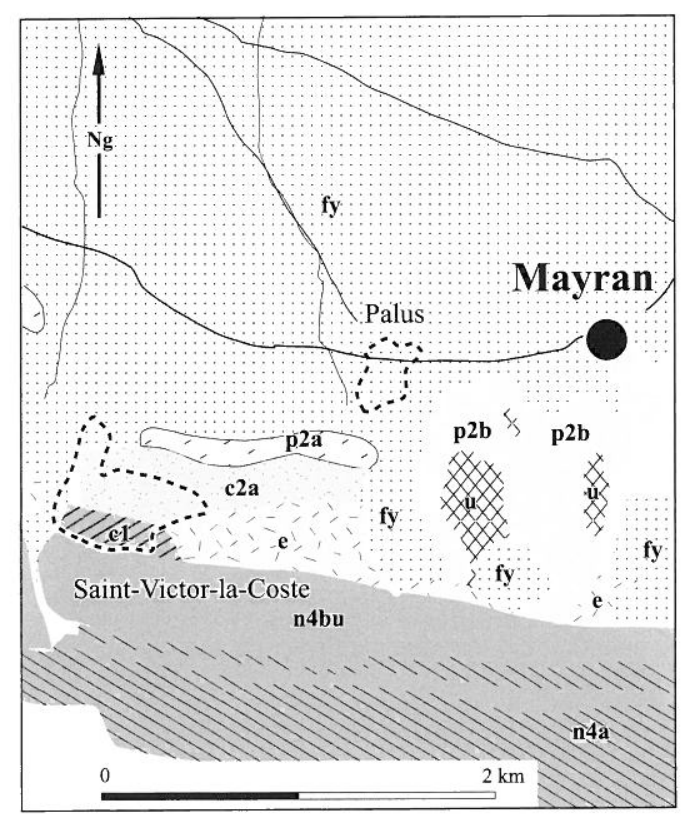

Fig. 4. Le site de Mayran dans son contexte géologique (d'après la carte géologique).

$\mathrm{u}$ : tufs quaternaires, e : éboulis, $\mathrm{fz}$ : alluvions modernes, fy: terrasses würmiennes, p2b: Pliocène astien, p2a: Pliocène plaisancien, c1: Albien Vraconien (sables roux et grès), c2a: Cénomanien inférieur, $\mathrm{n} 4 \mathrm{a}$ : Barrémien inférieur, $\mathrm{n} 4 \mathrm{bu}$ : Urgonien.

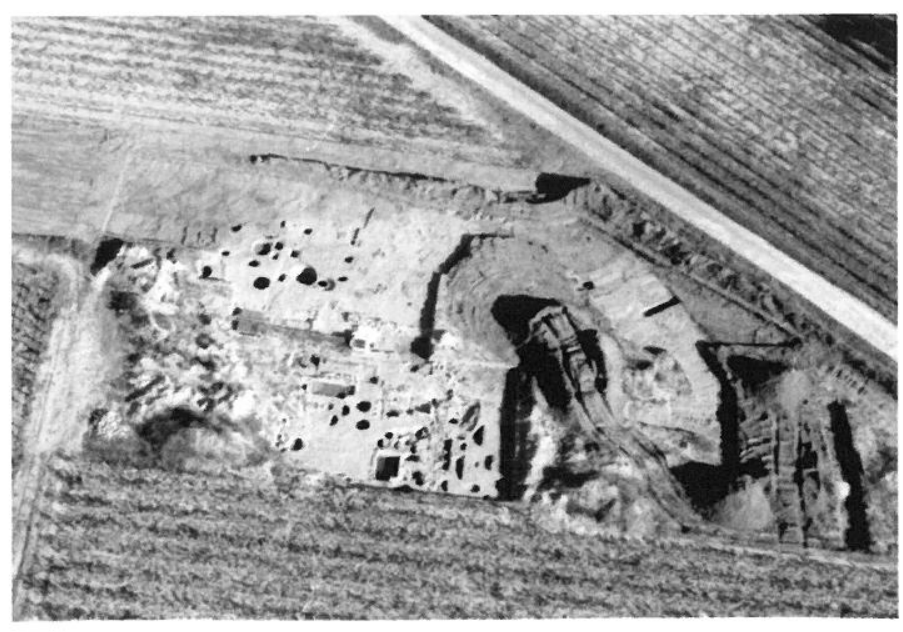

Fig. 5. Vue aérienne du chantier de fouille (mai 1996) (cliché P. Druelle).

\subsubsection{Les textes médiévaux et modernes}

L'histoire de Mayran durant l'époque médiévale est documentée par plusieurs textes, dont E. Pélaquier a assuré l'étude. La plus ancienne mention du site figure dans une charte de 896 , où Louis l'Aveugle accorde à Amiel, évêque d'Uzès, la possession de nombreux biens, parmi lesquels l'église de Saint-Martin à proximité du vieux Mayran (Sancti Martini ad Marianum veterem, Gallia Christiana, 4, col. 293). D'autres mentions, plus tardives, font suite à celle de 896. En 1113-1114, l'église est citée dans une bulle de Pascal II sous le terme $S$ Maria de Mirano. Mayran fait alors partie des possessions de Saint-Victor de Marseille rattachées au diocèse d'Avignon (in episcopatu Avinionensi, Clément 1989, 156). Une bulle de Jean XXII, datée du 6 septembre 1316, mentionne l'attribution d'un bénéfice ecclésiastique à un certain Guillaume de Mayran, clerc du diocèse d'Uzès. Au $\mathrm{XVI}^{\mathrm{e}}$ siècle, on apprend que le prieur de Mayran possédait environ cinquante hectares. Les prieurs quittèrent le site au début du XVIII ${ }^{\mathrm{e}}$ siècle. Mayran fut alors habité par des ermites ne possédant plus que quelques lopins de terre.

\subsection{Le contexte archéologique: habitat et occupation du sol dans la vallée de la Tave (fig. 6)}

Comme nous l'avons souligné précédemment, la vallée de la Tave a fait l'objet de prospections intensives qui permettent de resituer l'établissement de Mayran dans un environnement plus large. Ces travaux offrent une base documentaire encore incomplète, mais qui permet ici de proposer une analyse préliminaire.

Il est indispensable de dire en préambule quelques mots sur les agglomérations romaines connues dans la vallée de la Tave. Deux oppida ont fortement marqué 

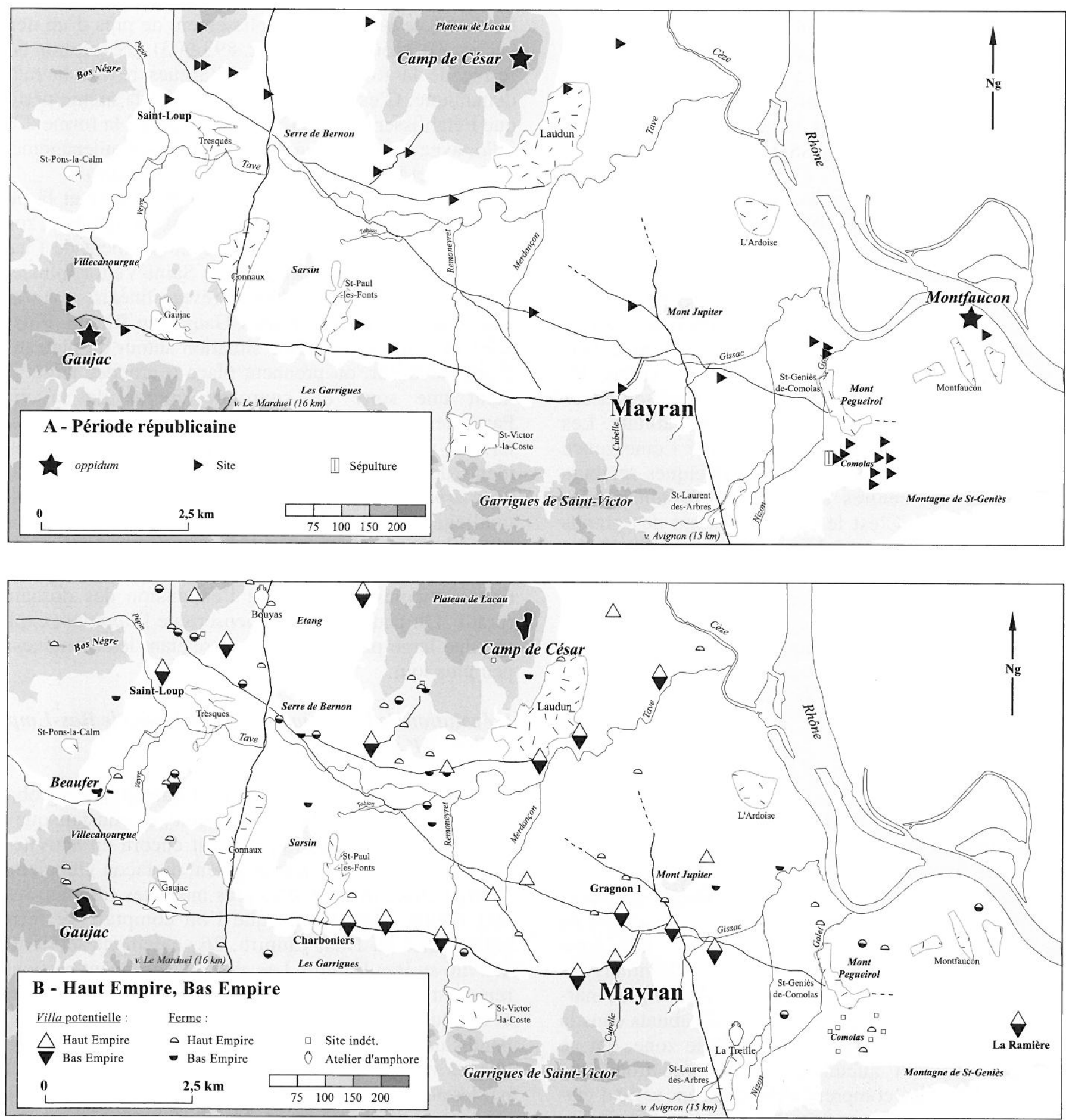

Fig. 6. L'occupation du sol à l'époque gallo-romaine dans la basse vallée de la Tave.

Ces cartes ne présentent que l'état actuel des recherches. II faut rappeler que les prospections n'ont pas eu un caractère systématique. Certaines lacunes des cartes archéologiques sont susceptibles d'être comblées par de nouvelles prospections. 
l'histoire de cette micro-région: à l'ouest, Gaujac, et à l'est, Le Camp de César (Laudun). Deux autres oppida sont connus: en bordure orientale de la vallée, Montfaucon, et au nord, Saint-Pierre-de-Castres. Mais ceux-ci n'ont pas joué sur le long terme un rôle aussi déterminant que Gaujac et le Camp de César.

\section{Prémices de l'occupation gallo-romaine: la période républicaine (fig. $6 \mathrm{~A}$ )}

Durant la période républicaine, la vallée de la Tave est dominée par les oppida du Camp de César, de Gaujac et de Montfaucon. En plaine, une quarantaine de petits établissements se développent (fig. 6A). On les trouve dans des secteurs particuliers. Les versants et le piémont des oppida de Gaujac et du Camp de César apparaissent assez logiquement comme des zones riches en habitats. Les agglomérations ont apparemment stimulé l'émergence d'un habitat de plaine précoce. Mais quelques secteurs plus éloignés des centres urbains sont également des zones dynamiques. C'est le cas en piémont des grands reliefs, par exemple au pied des garrigues de Saint-Victor ou à Tresques. La région de Saint-Geniès-de-Comolas livre également de très nombreux sites de cette période. Mais, la très forte densité des points de peuplement résulte ici des prospections systématiques qui ont été réalisées dans ce secteur. De vastes zones demeurent en revanche vides d'habitat, en particulier la partie centrale de la vallée.

\section{Le Haut-Empire (fig. 6B)}

Le Haut-Empire est marqué sans surprise par un net accroissement du nombre de sites qui, de 40 à la période républicaine, passe à 112 durant le Haut-Empire. La trame de peuplement antérieure qui ne couvrait que partiellement le territoire, est désormais renforcée par la mise en place de nouveaux établissements: fermes, villae, ainsi qu'une possible agglomération à Beaufer/Dominargues (Buffat et al. 2003, 232-236). Le phénomène le plus marquant est certainement la mise en place d'habitats dans la partie centrale de la plaine tavienne. Cette zone, qui ne livrait pratiquement aucune trace d'occupation à la période républicaine, comprend désormais une série d'établissements qui assurent sa mise en culture.

Dans cet essor de l'habitat rural, les villae jouent un rôle important. On recense effectivement 25 villae potentielles à la fin du $\mathrm{I}^{\mathrm{er}}$ ou au début du $\mathrm{II}^{\mathrm{e}} \mathrm{s}$. En rêgle générale, il est difficile de fixer avec précision la période de mise en place des villae créées ex-nihilo à cette période. On note cependant que parmi ces nouvelles créations peu livrent des éléments augustéens.

La villa de La Ramière illustre bien la montée en puissance de l'habitat tavien au cours du ${ }^{\mathrm{er}} \mathrm{s}$. La petite ferme du début du Ier s. ap. J.-C., fait place dans la seconde moi- tié du siècle à un vaste établissement de près d'un demihectare (Barberan et al. 2002, 893-903). L'évolution de la ferme de la Roquette à Cavilllargues révèle la même dynamique. C'est probablement aussi à la même période que l'établissement des Charboniers prend la forme d'une villa, avec son triclinium mosaïqué et ses aménagements agricoles et hydrauliques (Esteban et al. 2000).

Le piémont des garrigues de Saint-Victor et la périphérie du Camp de César apparaissent comme deux zones particulièrement favorables au développement de l'habitat. En piémont des garrigues de Saint-Victor, plusieurs villae sont organisées selon un réseau linéaire qui s'articule autour de la voie reliant Gaujac au Rhône. Plus au nord, on retrouve la même situation autour du plateau du Camp de César, où prennent place 6 grandes villae formant une sorte de couronne autour de la ville. Parallèlement, les versants du plateau de Lacau, avec leurs terroirs plus morcelés, sont occupés par des établissements de petite dimension, ne livrant pas d'éléments de pars urbana. Vers l'ouest, dans la plaine de Tresques, le maillage des villae apparaît également très dense. En revanche, en piémont de l'oppidum de Gaujac on ne connaît pas de villae. C'est peut-être ici l'exiguiité des terroirs agricoles qui a limité l'expansion des domaines ruraux. On peut également penser que l'oppidum exploitait une large part de ce terroir, rejetant les domaines un peu plus loin.

\section{L'évolution de l'occupation du sol durant le Bas-Empire (milieu III ${ }^{e}$ s.-V ${ }^{e}$ s.) (fig. 6B)}

Au Bas-Empire, le réseau de 1'habitat reste globalement stable. Si l'on observe les villae, on constate que sur les 25 occupées au II ${ }^{\mathrm{e}}$ s., 19 sont encore en activité au $\mathrm{IV}^{\mathrm{e}} \mathrm{s}$. Alors que les villae évoluent de façon globalement positive, les établissements plus modestes (fermes) résistent moins bien. Tandis que l'on comptait 49 fermes potentielles au Haut-Empire, 26 seulement seraient en activité au Bas-Empire. Les abandons frappent particulièrement trois secteurs : la plaine entre Saint-Victor-la-Coste et Laudun, le versant nord de l'oppidum de Gaujac et les pentes de l'oppidum de Camp de César. Il est manifeste que les versants des oppida sont des secteurs très touchés par les abandons. Mais on ne sait dire si l'exiguïté des terroirs agricoles est responsable de cette évolution, ou si c'est la perte de dynamisme des agglomérations qui en est la cause (Gaujac serait abandonné à la fin du III ${ }^{\mathrm{e}}$ s., alors que le Camp de César perdure dans une certaine atonie aux III ${ }^{e}-V^{e}$ s.). Dans la plaine de Tave, ce sont surtout les établissements installés dans des terroirs agricoles difficiles qui sont victimes des abandons. Il apparaît que les terroirs encombrés de gelifracts calcaires ou de galets sont particulièrement touchés par les désertions, entre SaintVictor et Laudun tout autant que vers Saint-Geniès-deComolas. Ce phénomène touche les villae et les fermes. 


\subsection{Les opérations archéologiques réalisées entre 1995 et 2002}

\subsubsection{La fouille de 1995-1997: stratégie de l'intervention}

La fouille de sauvetage conduite sur le site entre 1995 et 1997 a été menée préalablement à la remise en culture d'une parcelle située à l'est du site (AH 73 est). Des sondages de diagnostic effectués entre février et avril 1995 révélèrent un arasement prononcé des structures sur l'ensemble de la parcelle. Partout affleurait le substrat pliocène sableux. Seuls les aménagements en creux (fosses, fondations) étaient conservés. Ce fort arasement nous a donc incités à explorer la zone menacée en deux fenêtres de décapage de dimension moyenne (fig. 7). $1130 \mathrm{~m}^{2}$ ont été fouillés in extenso jusqu'en $1997^{1}$.

Le chantier a été divisé en deux zones géographiquement distinctes (zone 1 et 2, fig. 6). La zone 1 correspond à la partie sud de la parcelle. Elle couvre une superficie de l'ordre de $1000 \mathrm{~m}^{2}$. Pour des raisons d'ordre pratique, elle a été scindée en trois secteurs (1, 2 et 3, fig. 6). C'est dans cette zone que se concentrent la plupart des aménagements: installations vinicoles du Bas-Empire, aqueduc souterrain, silos médiévaux. La zone 2 , beaucoup plus modeste, occupe l'extrémité nord de la parcelle. Elle s'étend sur environ $130 \mathrm{~m}^{2}$. L'exploration de cette zone a été motivée par la découverte dans les années 1950, de constructions par M. Faraud. Il s'est avéré que ces constructions étaient en relation avec l'aqueduc souterrain, découvert plus tard par L. Faraud.

L'enregistrement de la documentation archéologique a été réalisé selon les protocoles utilisés par l'équipe de Lattes. Les descriptions d'unités stratigraphiques ont été enregistrées sur base informatique Filemaker Pro, de même que les inventaires de mobilier.

\subsubsection{La prospection méthodique de 2002}

Les fouilles n'ont permis d'appréhender qu'une part très limitée du site. Sur une concentration de mobilier romain qui s'étend sur 4,5 ha, seulement 0,15 ha ont été décapés. Il est apparu nécessaire de compléter cette fouille par une prospection fine. Celle-ci devait analyser finement l'ensemble du site et tenter de discerner quelques grandes lignes de son évolution ${ }^{2}$.

Cette prospection a été conduite en deux phases. La première a consisté à relever les densités d'artefacts en surface, la seconde à prélever du mobilier céramique à tra-

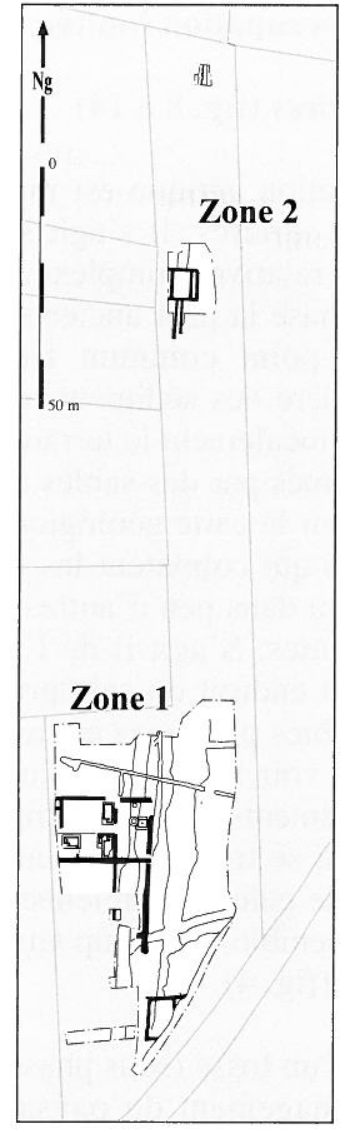

Fig. 7. Plan du chantier de fouille avec localisation des zones et secteurs. vers une série de tests de ramassage de $100 \mathrm{~m}^{2}$. Vingtdeux tests ont été effectués, permettant de dégager quelques tendances générales quant à l'évolution du site. Les résultats de ces tests seront présentés conjointement à ceux de la fouille. L'inventaire du mobilier collecté à cette occasion est présenté en annexe.

\section{LES PREMIÈRES TRACES D'OCCUPATION ANTIQUE} (phase B1, Ir s. av. J.-C. - début du $\mathrm{I}^{\mathrm{er}}$ s. ap. J.-C.)

Après une occupation à l'âge du Bronze (sépultures, fosses), dont la présentation serait hors de propos, le site de Mayran est à nouveau occupé à partir du $\mathrm{I}^{\mathrm{er}} \mathrm{S}$. av. J.-C. Cette période n'est représentée dans le cadre de la fouille que par quelques aménagements agraires: un chemin, plusieurs fossés sont les rares éléments de cette occupation ancienne. Les prospections indiquent que l'installation principale de cette période prend place au sud-ouest du chantier. Autant que l'on puisse en juger, il s'agit d'un habitat modeste.

1 La fouille s'est déroulée sous la forme de plusieurs stages de courte durée, organisés en collaboration avec différentes associations. L'équipe d'encadrement était constituée de L Buffat et H. Petitot. Plusieurs personnes ont contribué à l'encadrement des équipes de bénévoles qui se sont succédé sur le terrain: J. Guerre, A. Avril, Chr. Soler, M. Gomez, A. et B. Istas, R. Platon, A. Raux, L. Vidal.

2 Cette prospection a été menée les 13 et 14 juin 2002 par une équipe constituée de P. Caubisens, I. Commandré, C. Curinier, M. Mendiboure, Chr. Soler, N. Viala et L. Buffat. 


\subsection{Quelques témoins d'une occupation limitée}

\subsubsection{Des aménagements agraires (fig. 8 à 14)}

La première phase d'occupation antique est marquée par une série d'aménagements agraires. Il s'agit surtout de fossés, dont la chronologie relative, complexe, attestent différentes étapes dès la phase la plus ancienne. Ces aménagements possèdent un point commun frappant constitué par la nature particulière des sédiments qui les remplissent. En effet, alors que localement le terrain naturel et le sol labouré sont constitués par des sables astiens de couleurs jaunes (notés p2b sur la carte géologique), ce sont des limons sableux rouges qui colmatent les fossés. On retrouve ce type de sédiment dans peu d'autres structures archéologiques plus récentes. S'agit-il de l'ultime témoignage de l'existence à cet endroit du sol "primitif" qui s'était développé sur ces sables pliocènes au début de l'Holocène? Ou bien faut-il y voir la marque d'un flux ayant prélevé par érosion les éléments fins des formations sédimentaires superficielles, qui se trouvent tout autour? En l'occurrence des terrasses de galets würmiennes dont la matrice sablo-limoneuse ressemble beaucoup aux sédiments qui colmatent les fossés (fig. 4).

- Première étape : creusement d'un fossé (sous phase B1a)

Le premier élément d'aménagement du paysage est constitué par un fossé, repéré en sondage au sud du chantier (fig. 11). Tout en étant le vestige le plus ancien, il présente au moins deux états. Le plus ancien (1395), au large profil irrégulier, voit son colmatage recoupé par un autre creusement, très modeste, à fond plat et parois sub-verticales (1389). On ne peut en dire plus sur cet élément observé seulement dans un sondage, et qui a en outre été dégagé sur une trop faible longueur pour que l'on puisse déterminer avec précision son orientation. Cependant, il faut remarquer que le fossé 1395 pourrait posséder une direction nord-sud que l'on retrouvera plus tard dans celle du fossé 1381 mis en place dans la troisième étape (voir plus bas) et qui s'y superpose (fig. 12 et 14).

- Deuxième étape : installation d'un chemin et d'un fossé (sous phase $\mathrm{B} 1 \mathrm{~b}$ )

Une seconde étape dans la structuration du paysage est marquée par l'installation d'un chemin et d'un fossé (1373, fig. 8 et 9). De direction est-ouest, le chemin recoupe presque perpendiculairement le fossé 1395-1389 (fig. $11,12, \mathrm{n}^{\circ} 1$ ). Il a connu deux phases de fonctionnement distinctes (fig. 14): la première est caractérisée par un niveau compact de graviers (1388), la seconde par une épaisse recharge de cailloutis calcaire (SL 1378), au sommet de laquelle une ornière a été distinguée. Large d'environ $2 \mathrm{~m}$, le chemin présente une orientation ( $\mathrm{NG} 4^{\circ} \mathrm{W}$ ), qui ne se rattache à aucune des centuriations proposées dans la région. Cependant, elle se rapproche d'une orien-

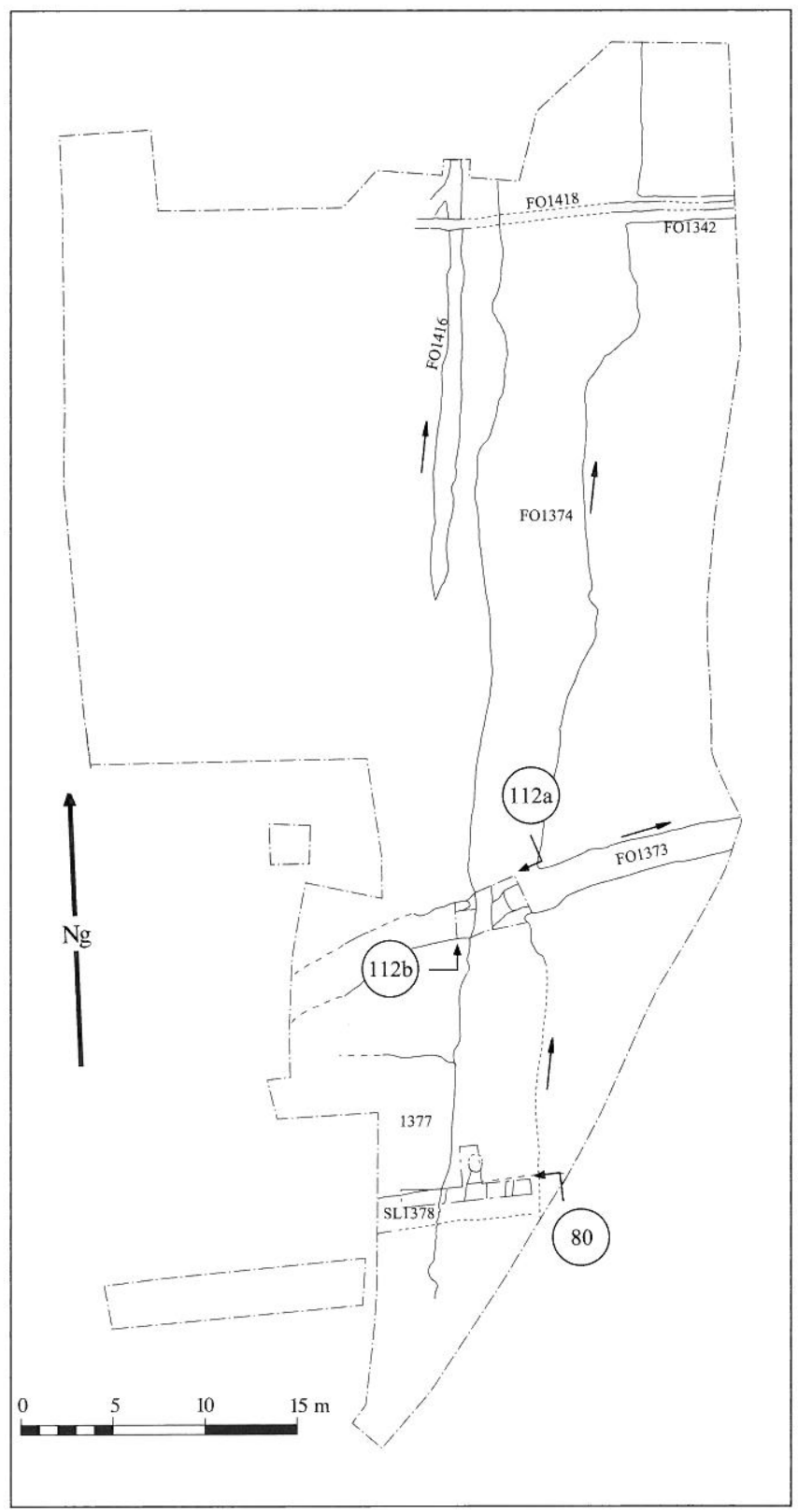

Fig. 8. Plan cumulé des aménagements de la phase B1.

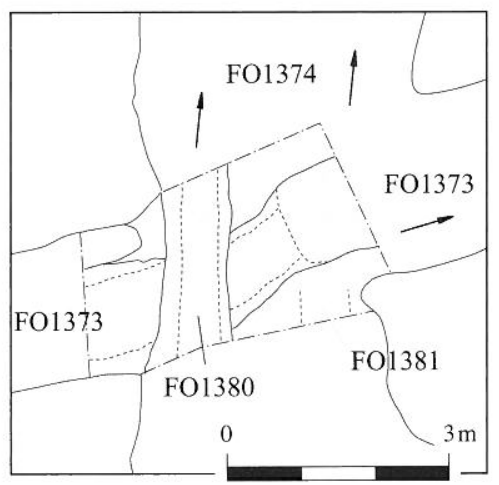

Fig. 9. Relevé de l'intersection entre les fossés 1373 et 1380 . 


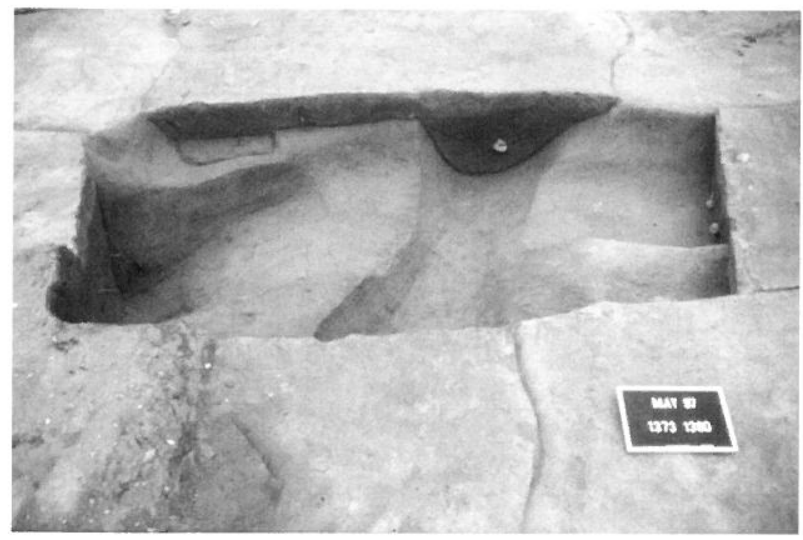

Fig. 10. Vue de l'intersection entre les fossés 1373 et 1374 (cliché L. Buffat).

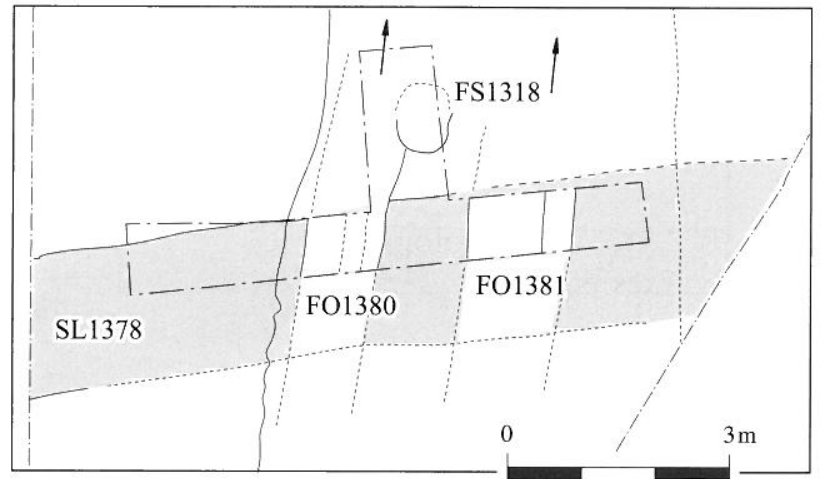

Fig. 11. Le chemin 1378, recoupé par les fossés 1380 et 1381.

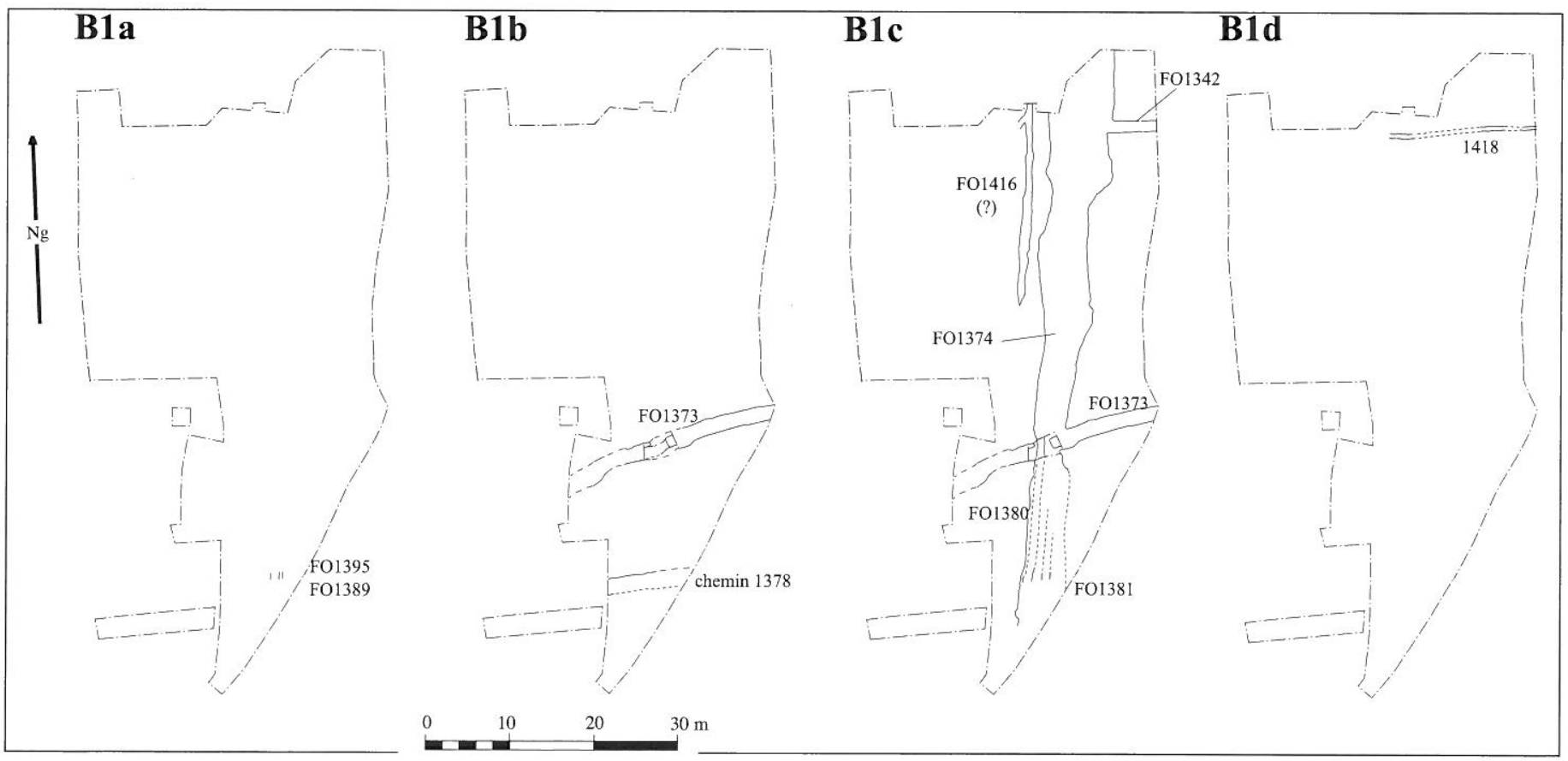

Fig. 12. Évolution des aménagements de la période B1.

tation bien représentée dans la vallée de la Tave (NG $5^{\circ}$ $\mathrm{W}$ ) et que M. Assénat et $\mathrm{A}$. Pérez interprètent comme les traces d'un vaste cadastre, qui se développerait de Nîmes à Orange (Assénat, Pérez 1997). Toutefois, la réalité de cette centuriation d'extension est-ouest reste très discutée (Favory 1997, 98-102).

À une quinzaine de mètres au nord du chemin se trouve un fossé (FO1373, fig. 8, 9), peu profond dont le profil garde la trace de deux creusements successifs. Ce fossé présente une orientation de $\mathrm{NG} 13^{\circ} 8 \mathrm{~W}$, qui se rapproche de celle du réseau Nîmes $\mathrm{B}\left(\mathrm{NG} 13^{\circ} \mathrm{W}\right)$ ou de l'Orange D ou E. Ce fossé ne possède pas de lien physique avec le chemin, mais on notera que ces deux structures sont recoupées par le même fossé (FO1374).
- Troisième étape : mise en place d'un réseau (sous-phase B1c)

Le décapage a permis de dégager, dans la partie est de la zone de fouille, ce qui semblait être un grand fossé nord-sud (1374) venant se greffer ou recouper le fossé 1373, alors partiellement comblé (fig. 12, B1c). L'emprise de la fouille n'a pas permis de déterminer l'extension de ce nouveau fossé. Les deux sondages réalisés montrent que l'image de surface d'un fossé, dont la largeur irrégulière oscille entre $8 \mathrm{~m}$ au nord puis $6 \mathrm{~m}$ au milieu et enfin $5 \mathrm{~m}$ au sud, est en fait le résultat de la coalescence de plusieurs creusements isoclines. Ainsi, sans que les sections réalisées révèlent de façon nette tous les rapports chronologiques qui les lient, il faut distinguer un large creuse- 


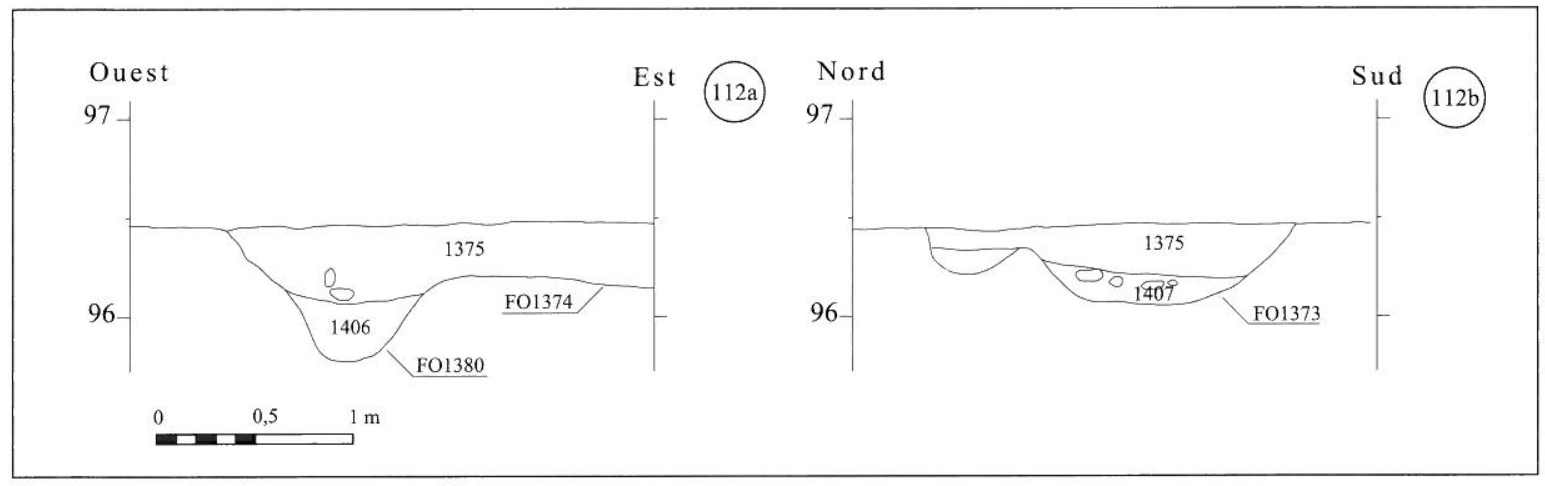

Fig. 13. Coupes des fosses 1373 et 1380 .

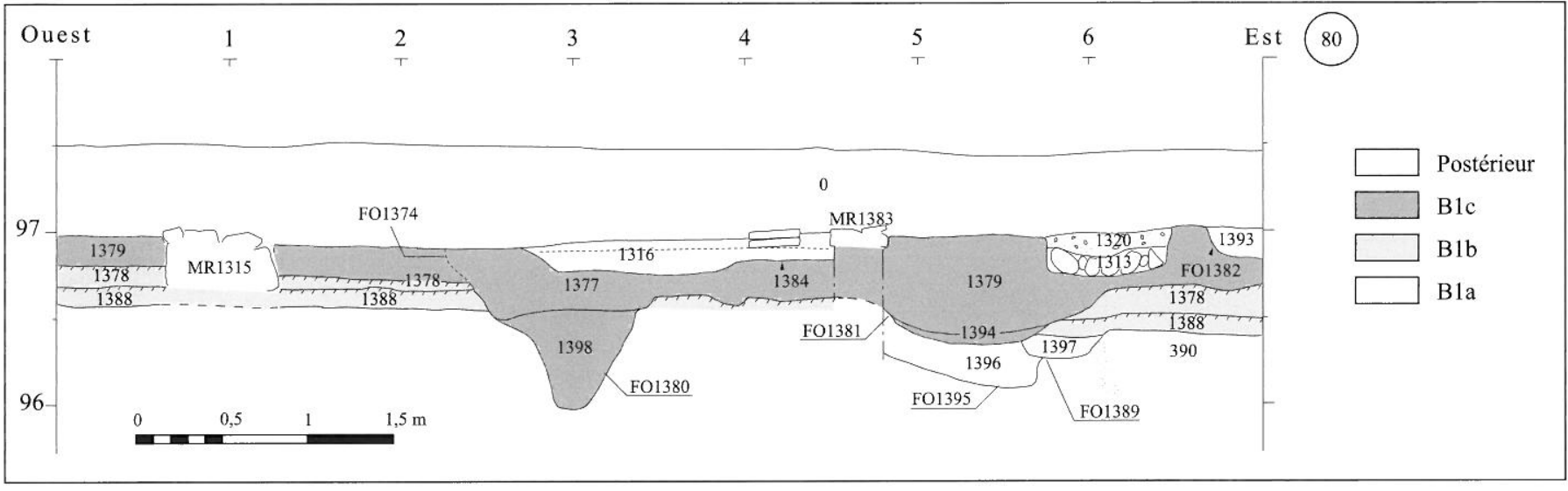

Fig. 14. Coupes des aménagements agraires de la phase B1, reconnus sous un bâtiment postérieur.

ment peu profond au fond plat, et au moins deux fossés étroits ${ }^{3}(1380,1381$, fig. 11). Ces petits fossés sont assez différents l'un de l'autre. Celui qui se trouve à l'est est peu profond $(0,35 \mathrm{~m})$ et comporte des parois très évasées, alors que celui observé à l'ouest (1380) est plus profond $(0,75 \mathrm{~m})$ et présente des bords plus droits (fig. 14). L'entretien du dispositif est révélé par des traces de curage, perceptibles notamment au niveau du fossé 1381 . Ce nouveau système de fossés présente une orientation de NG 6-7 ${ }^{\circ}$ E, qui se rapproche de celle du cadastre B d'Orange $\left(\mathrm{NG} 5^{\circ} \mathrm{E}\right)$. À l'extrémité nord du faisceau de creusements (FO1374) se trouve un diverticule (1342) qui n'est pas absolument perpendiculaire $\left(\mathrm{NG} 1^{\circ} \mathrm{W}\right)$ mais qui semble bien fonctionner avec tout ou partie du faisceau de creusements.

Un autre élément peut se rattacher à ce groupe. En effet, à l'ouest du grand fossé central, se trouve un fossé plus modeste (1416) se développant en parallèle à ce dernier. Dégagé sur une vingtaine de mètres, il est relié au nord à un autre fossé dont on ignore l'extension, puisqu'il a été recoupé par plusieurs aménagements postérieurs. Il n'existe pas de lien fonctionnel avec le réseau, et seul le fait qu'il soit recoupé par le même fossé (voir ci-dessous) nous invite à les regrouper. Mais reconnaissons, qu'il pourrait tout aussi bien appartenir à une sous-phase précédente.

- Quatrième étape : abandon progressif du réseau de fossé (sous-phase B1d)

Le fossé 1418 constitue l'aménagement le plus tardif (fig. 12, n 3). À l'époque où il est creusé, le système fossoyé antérieur est abandonné et totalement comblé par des colluvions. Ce nouveau fossé (non fouillé) reprend la même orientation que le fossé 1342 soit $1^{\circ} 1 \mathrm{~W}$.

\section{- Datation}

Le matériel fait défaut pour dater précisément les différentes étapes de cette première occupation antique. Outre des tessons centimétriques de céramique non tournée, quelques fragments d'amphore italique sont présents dans certains comblements. Ces témoins invitent à dater le fonctionnement de ces aménagements d'un large $\mathrm{I}^{\text {er }} \mathrm{s}$.

3 La disposition n'est pas sans rappeler un précepte de Palladius $(1, \mathrm{XXXIV}, 6)$ : «Alors, à l'endroit où doit se dresser la haie, on creusera, à 3 pieds l'un de l'autre, deux sillons d'un pied et demi de profondeur [...] Au bout de trente jours, les ronces sortent de terre ». 
av. J.-C. Le comblement des grands fossés 1374 et 1373, qui marque l'abandon de l'essentiel du réseau fossoyé, livre quelques éléments du début du Haut-Empire (non tournée bitumée, kaolinitique, amphore gauloise). Ce mobilier situe le terminus ante quem de cette phase de mise en valeur de la campagne durant la première moitié du Irr s. ap. J.-C.

\section{INVENTAIRE DU MOBILIER}

1378 - amphore indéterminée: 1 fr.

$1375(-50 / 0)$ phase B1 - non tournée: 3 fr., non tournée bitumée: $3 \mathrm{fr}$, commune italique: $1 \mathrm{fr}$.

$1379(0 / 25)$ phase B1 - kaol. oxydante: 1 fr., kaol. réductrice : 2 fr., non tournée bitumée : 5 fr., amphore gauloise sabl.: 2 fr., amphore de Bétique: $1 \mathrm{fr}$.

$1406(-50 / 0)$ phase B1 - non tournée: 1 fr., amphore italique : 1 fr.

$1417(-50 / 0)$ phase B1 - non tournée: 6 fr. (CNT-LOR U2)

$1420(0 / 50)$ phase B1 - kaolinitique oxydante: $1 \mathrm{fr}$.

\subsubsection{Conclusions}

Si elle reste ponctuelle, la découverte d'aménagements agraires républicains et augustéens n'en demeure pas moins intéressante. Elle est d'abord révélatrice de l'ancienneté de l'occupation antique sur le site de Mayran. Elle atteste, à travers les différents réaménagements, une mise en valeur soutenue et durable du terroir. Elle livre aussi des éléments qui invitent à s'interroger sur le problème de la cadastration et de la morphologie agraire antique dans cette partie de la vallée de la Tave.

Ces aménagements parcellaires témoignent d'une organisation complexe du paysage où coexistent plusieurs orientations. À ce titre, Mayran vient renforcer les observations effectuées sur le site voisin de La Ramière (Barberan et al. 2002), où les plantations, les chemins ou les haies répondent à des orientations multiples. Ces exemples mettent en garde sur une restitution trop simple de l'organisation des parcellaires, du moins dans le secteur concerné par ces deux sites. Il apparaît possible que le cadastre Orange B ait eu un impact sur le parcellaire de Mayran, comme en témoignent plusieurs fossés. L'extension de ce cadastre dans la vallée de la Tave a déjà été proposé (Chouquer 1994, 51). Mais d'autres limites, d'orientation différentes existent, soit plus anciennes, soit plus récentes.

Il reste difficile de dépasser ces premiers constats. Aller plus loin nous entraînerait vraisemblablement vers une surinterprétation des données ou conduirait à leur attribuer plus de valeur qu'elles n'en ont réellement. Localement, des progrès dans le domaine de la morphologie parcellaire antique ne pourront être enregistrés que par de nouvelles fouilles.

\subsection{Données de prospection: une occupation républi- caine probablement très limitée}

En prospection de surface, la période républicaine se signale par quelques tessons d'amphore italique et de céramique non-tournée repérés dans deux concentrations, situées au contact sud-ouest de la fouille (fig. 21). Ces éléments restent discrets et témoignent peut-être d'une occupation limitée, habitat de petite dimension et/ou structures agraires (fossés, fosses). Mais il est aussi possible que cette occupation ancienne soit sous-représentée en surface, du fait de l'occupation postérieure.

\section{LE HAUT EMPIRE: LES PREMIERS BÂTIMENTS DE LA VILLA}

(phase B2, $\mathrm{I}^{\mathrm{er}}-\mathrm{III}^{\mathrm{e}}$ s. ap. J.-C.)

Comme c'est souvent le cas sur les établissements ruraux de la région, les signes d'occupation se multiplient à Mayran à partir de la seconde moitié du I ${ }^{\mathrm{er}} \mathrm{s}$. ap. J.-C. La fouille n'a que peu appréhendé la morphologie de l'établissement du Haut-Empire, dont le cœur se situe, d'après les prospections de surface, $100 \mathrm{~m}$ au sud-ouest de la fouille. Là se développe sur près d'un hectare une zone à forte concentration de mobilier, qui livre différents éléments résidentiels (tubuli, tesselles). Autour d'elle, des petites concentrations prennent place. On propose de voir dans cet ensemble les traces d'une première villa. Dans l'emprise de la fouille, un seul bâtiment du Haut-Empire a été appréhendé. On ne sait rien de sa fonction initiale. Il sera équipé plus tardivement, peut-être au $\mathrm{III}^{\mathrm{e}} \mathrm{s}$., d'un hypocauste à canaux.

\subsection{Le bâtiment sud (fig. 15 à 18)}

\subsubsection{La mise en place du bâtiment}

C'est au sud du chantier qu'a été repéré le plus ancien bâtiment dégagé lors de la fouille (fig. 15). On a peu de renseignements sur ce bâtiment qui s'étend au-delà des limites du chantier. De direction est-ouest, il comprend au moins deux pièces. L'une d'elles a été dégagée presque intégralement. De forme trapézoïdale, elle couvre une surface d'environ $35 \mathrm{~m}^{2}$. Cet aménagement est mal documenté, d'abord parce qu'il a été arasé par les labours, ensuite parce qu'il a été épierré à la fin de l'Antiquité. Les tronçons de murs qui subsistent font appel à des techniques de construction hétéroclites. Le mur 1315 correspond à une fondation, conservée sur 2 assises, constituée de blocs (calcaire urgoninen et grès tavien) liés à la terre. Le mur 1312 fortement épierré, comprend 2 premières assises constituées de blocs et de tuiles liés à la terre, 3 assises supérieures constituées de blocs de calcaires et de grès liés au mortier blanc. Le mur de cloison 1313, conservé sur une assise de fondation, est constitué de 

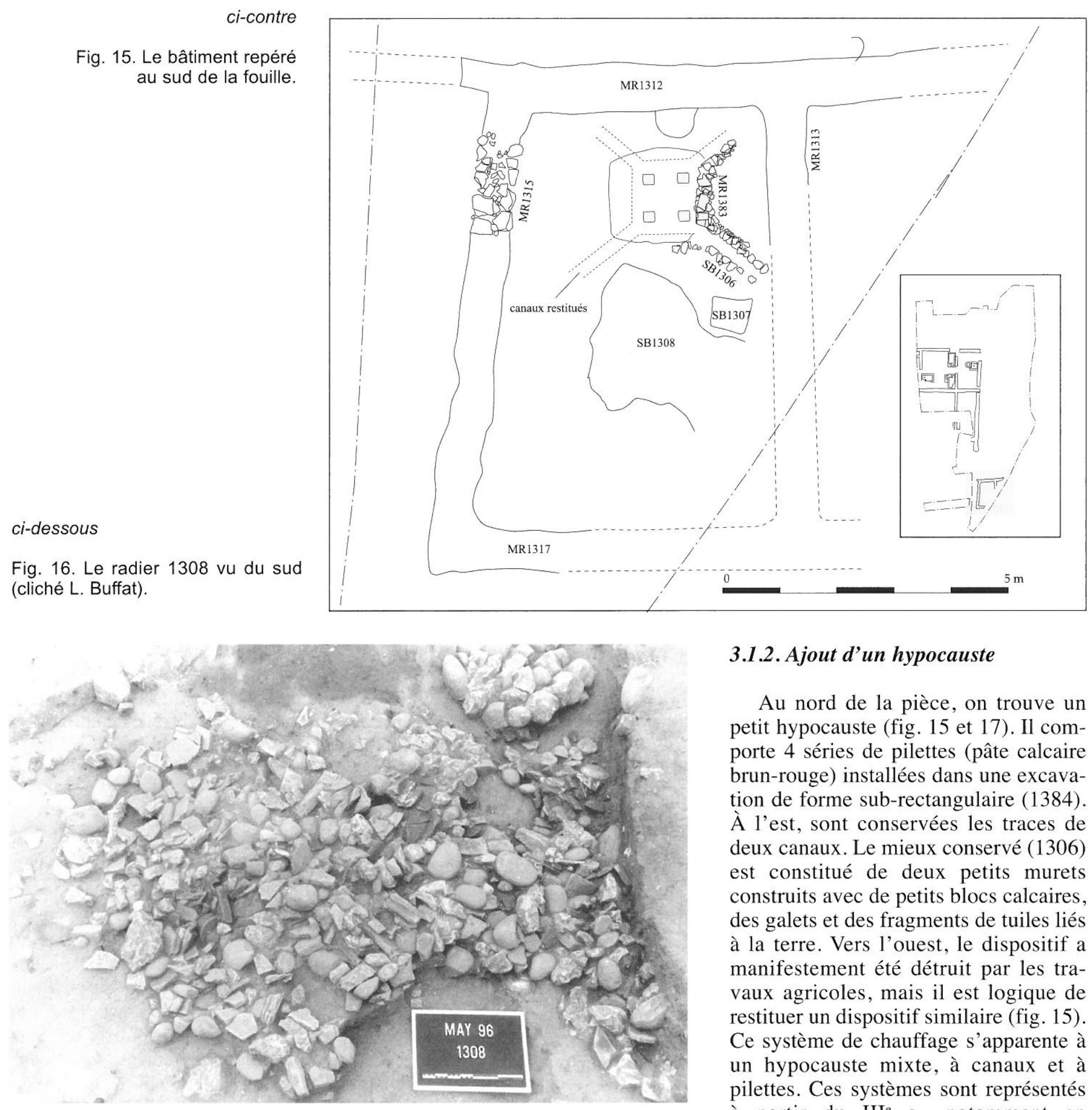

\subsubsection{Ajout d'un hypocauste}

$\mathrm{Au}$ nord de la pièce, on trouve un petit hypocauste (fig. 15 et 17). Il comporte 4 séries de pilettes (pâte calcaire brun-rouge) installées dans une excavation de forme sub-rectangulaire (1384). À l'est, sont conservées les traces de deux canaux. Le mieux conservé (1306) est constitué de deux petits murets construits avec de petits blocs calcaires, des galets et des fragments de tuiles liés à la terre. Vers l'ouest, le dispositif a manifestement été détruit par les travaux agricoles, mais il est logique de restituer un dispositif similaire (fig. 15). Ce système de chauffage s'apparente à un hypocauste mixte, à canaux et à pilettes. Ces systèmes sont représentés à partir du $\mathrm{III}^{e}$ s., notamment en Aquitaine. On en a très peu d'exemples

galets liés à la terre. Au sud du bâtiment, on trouve deux aménagements assez arasés: un radier de sol (1308) fait de matériaux divers (tuiles, galets, blocs calcaires, dolium et blocs de tuileau), et une base de pilier (1307), constituée de galets et de blocs calcaires liés à la terre. Le mobilier recueilli dans le radier 1308 , situe le fonctionnement du bâtiment entre la seconde moitié du $\mathrm{I}^{\mathrm{er}} \mathrm{s}$. et la fin du $\mathrm{II}^{\mathrm{e}} \mathrm{s}$. ( $c f$. inventaire). dans la région. Le seul qui soit clairement attesté en Languedoc a été reconnu sur la villa de Loupian. Durant le dernier état (IIIb), daté de la fin du IV eu du début du $\mathrm{V}^{\mathrm{e}} \mathrm{s}$., la partie résidentielle comprend une grande salle triconque. L'abside sud de ce triconque est chauffée par un hypocauste mixte (Pellecuer 2000, 489, fig. 51-11). En Provence, ce type d'hypocauste est connu sur l'établissement d'Eyguières (Bouches-du-Rhône), pour le chauffage 


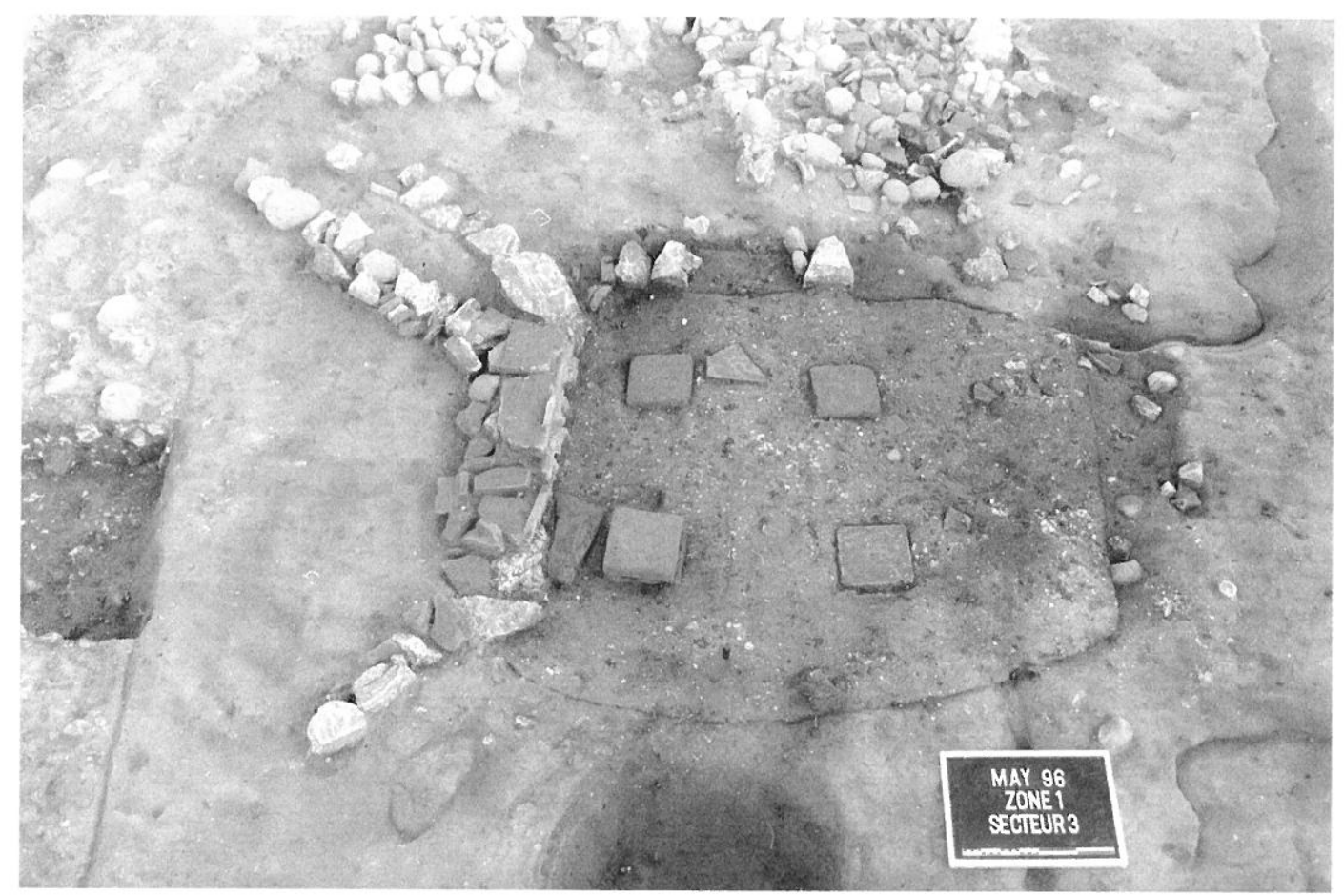

Fig. 17. L'hypocauste à canaux vu du nord (cliché L. Buffat).

d'une salle située au sud-est du grand balneum (Pelletier, Poguet 2000, 285, fig. 14, $\mathrm{n}^{\circ} 14 ; 287-288$ ).

Cet hypocauste est visiblement plus tardif que le bâtiment lui même. On a effectivement souligné qu'il appartenait à un type dont on situe l'apparition au $\mathrm{III}^{\mathrm{e}} \mathrm{s}$. Or, le mobilier trouvé dans le radier 1308 indique que le bâtiment fonctionnait déjà au Haut-Empire. L'hypocauste occupe par ailleurs une position désaxée à l'intérieur de la pièce, ce qui conforte l'hypothèse d'une construction postérieure. Malheureusement, les lacunes stratigraphiques empêchent de préciser dans le détail la chronologie de ces modifications.

L'arasement des vestiges ne permet pas de rentrer dans le détail de fonctionnement de cet hypocauste. Il serait assez logique de localiser le praefurnium à l'extérieur du bâtiment en l'occurrence au nord: les hypocaustes à canaux de la villa des Près-Bas (état IIIa) sont chauffés par des foyers situés à l'extérieur du bâtiment, de même que celui de l'abside sud du triconque de la villa IIIb (fig. 18). Concernant le système de chauffage des parois, l'utilisation d'un système avec tubuli est fort probable. Les fragments de tubuli sont en effet assez nombreux dans les contextes proches (tranchée d'épierrement du bâtiment, grande fosse 1130 située au nord du corps de bâtiment).

L'abandon du bâtiment intervient visiblement à une date assez tardive. Le comblement de l'hypocauste (1316) a livré des éléments datables de la fin du $\mathrm{IV}^{\mathrm{e}}$ ou du $\mathrm{V}^{\mathrm{e}} \mathrm{s}$. L'épierrement du bâtiment est daté, par quelques rares tessons de la fin du $\mathrm{V}^{\mathrm{e}} \mathrm{s}$. ou du $\mathrm{VI}^{\mathrm{e}} \mathrm{s}$.

D’une façon générale, il est difficile de conclure sur ce bâtiment, mal conservé et partiellement dégagé. Tout indique qu'il a fonctionné pendant plusieurs siècles et qu'il a subi des remaniements. Mais on ne peut rentrer dans le détail de son évolution. La présence d'un hypocauste à canaux confère à la pièce dégagée une vocation résidentielle. Mais cet aménagement, apparemment plus tardif traduit-il la vocation initiale du bâtiment?

\section{INVENTAIRE DU MOBILIER}

\section{Fonctionnement du bâtiment sud}

radier $1308(25 / 250)$ phase B2 - claire récente: 2 fr. (fond de mortier indéterminé, CL-REC 18a), kaol. réductrice: 1 fr., amphore gauloise: 15 fr., amphore gauloise sabl.: 6 fr. (2 anses de G1), amphore de Bétique: 1 fr. Total céramiques: $25 \mathrm{fr}$.

Abandon de l'hypocauste

1316 (300/450) phase C - claire B/luisante: 1 fr. (Portout 37a), claire engobée oxydante: 1 fr., claire récente: 2 fr., kaol. oxydante: 1 fr. (KAOL A3), kaol. réductrice: 9 fr. (KAOL A24), amphore de Bétique: 1 fr., amphore africaine: 1 fr., amphore indéterminée: $1 \mathrm{fr}$. Total céramiques : $17 \mathrm{fr}$.

os animaux : $9 \mathrm{fr}$.

\subsection{Un élément de bornage (fig. 19 et 20)}

Au nord du bâtiment, un autre aménagement est à rattacher au Haut-Empire. Il s'agit d'une petite fosse circulaire d'une trentaine de centimètres de diamètre, à l'intérieur de laquelle, plusieurs fragments d'une amphore gauloise sableuse (G1) étaient empilés (seul celui du fond se présentait par sa face interne). Un galet ovoïde blanc était disposé contre la paroi (fig. 20). 


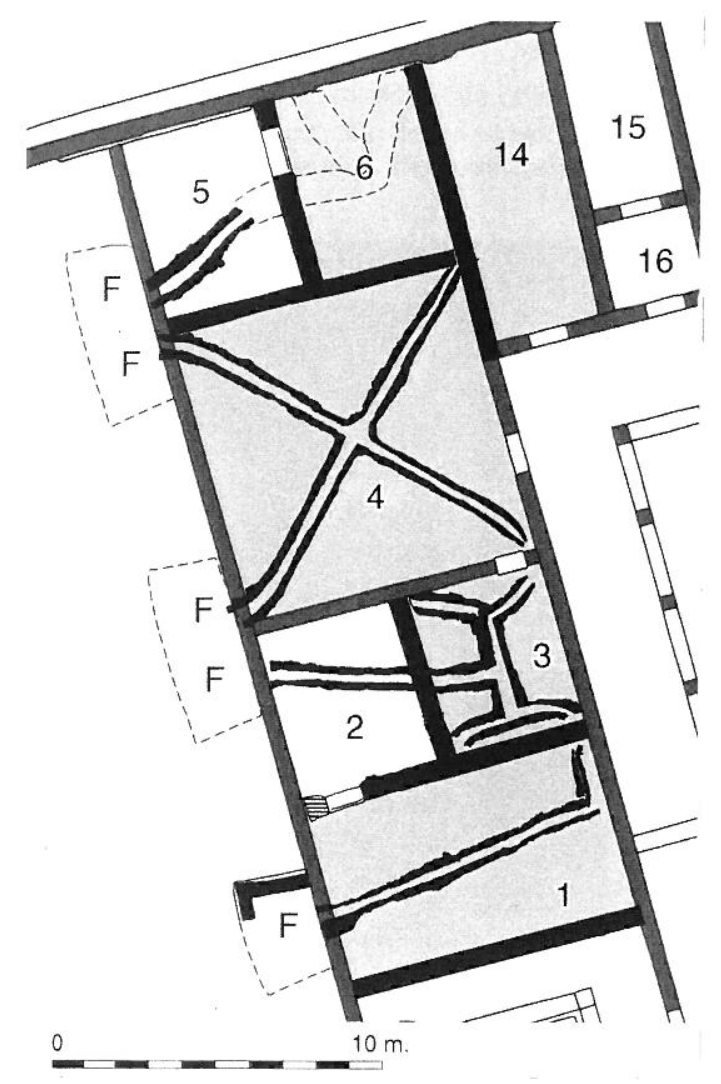

Fig. 18. Hypocaustes à canaux de la villa de Loupian. Les foyers sont situés à l'extérieur du bâtiment (marqués par un F) (Pellecuer 2000, 471, fig. 42-1).

Cet aménagement se situe à l'intersection de deux fossés plus anciens, désormais comblés (fig. 19) et pérennise ainsi une limite parcellaire ancienne. Il s'agit manifestement d'un élément de bornage, que les auteurs gromatiques appelaient terminus testacius (Vidal, Petitot 2003). S'agit-il d'une limite entre plusieurs propriétés? C'est envisageable, mais des travaux complémentaires devront le prouver ${ }^{4}$.

\subsection{Image générale du site au Haut-Empire: les données de la prospection}

Les prospections de surface apportent quelques éléments pour replacer dans un cadre plus général les rares structures du Haut-Empire exhumées lors de la fouille. Les prospections ont effectivement permis de repérer le principal pôle d'occupation du Haut-Empire, $100 \mathrm{~m}$ au sud-ouest des vestiges dégagés (fig. 21). Par rapport à la période républicaine, le Haut-Empire marque un net accroissement des surfaces occupées. La concentration principale se développe sur une surface de près d'1 ha. La

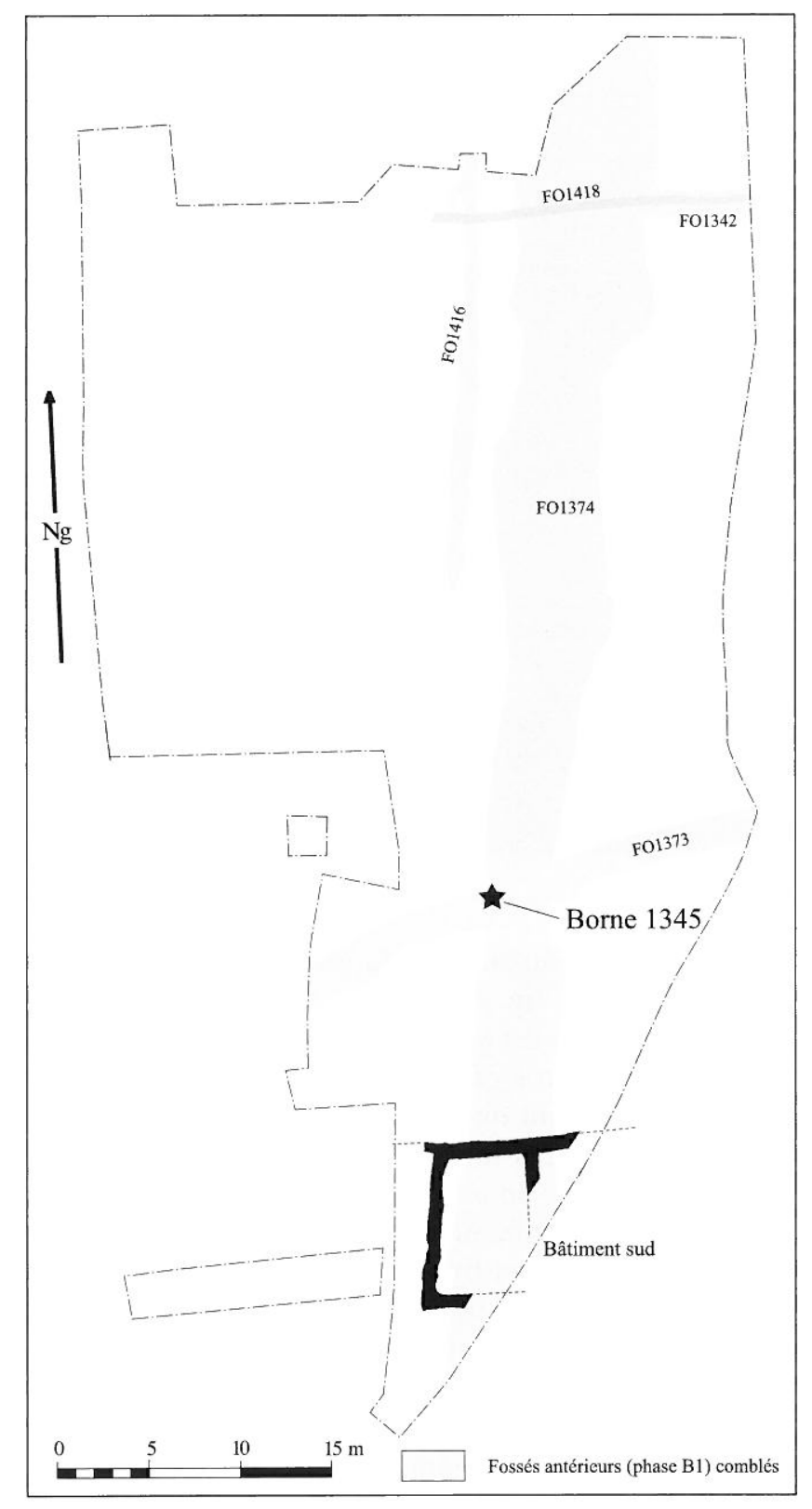

Fig. 19. Localisation de la borne 1345 implantée à l'intersection du réseau de fossés antérieurs, désormais comblé. La borne pérennise peut-être une limite de propriété.

prospection invite à localiser dans la partie sud de cette zone, un corps de bâtiment résidentiel, dans un secteur qui a livré une forte proportion de tubuli ainsi qu'une tesselle de mosaïque noire. On trouve également dans cette zone une proportion de céramiques fines nettement supérieure aux autres secteurs. Au nord et à l'est, les céramiques fines se raréfient, au profit des amphores.

4 Le dispositif de Mayran vient en tout cas compléter la liste des bornages trouvés dans la région. On citera notamment les exemples spectaculaires des têtes d'amphores plantées à l'envers comme sur le site de Sept Fonts à Saint-Pons-de-Mauchiens (Mauné 1998, 451-453) ou celui de La Peyrière à Lavérune (FORULA 1980). 


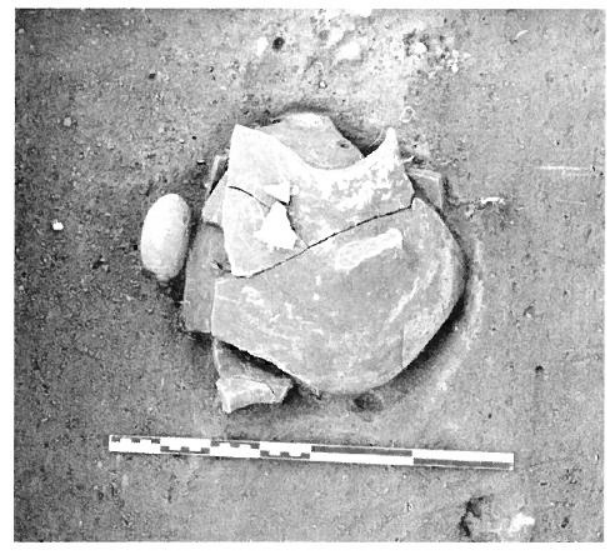

Fig. 20. Vue de l'élément de bornage 1345. Les fragments d'amphore ont été déposés les uns sur les autres dans une fosse de faible profondeur (cliché $\mathrm{H}$. Petitot).

Au sud-est de ce pôle principal, trois petites concentrations de 200 à $300 \mathrm{~m}^{2}$ marquent vraisemblablement l'emplacement de structures annexes (bâtiments, lieux d'activités agricoles ou aménagements fossoyés). Les céramiques recueillies situent leur fonctionnement entre le $\mathrm{II}^{\mathrm{e}}$ et le $\mathrm{III}^{\mathrm{e}} \mathrm{s}$. (sigillée sud-gauloise, claire B, amphore gauloise calcaire et sableuse). Au nord du chemin, une zone de 0,5 ha, livre des éléments assez diffus de cette période. Il reste difficile d'interpréter cette zone qui livre des proportions faibles de mobilier du Haut-Empire. S'agit-il d'une zone d'épandage en relation avec l'habitat? Existe-t-il quelques constructions de cette période, en relation avec le bâtiment exhumé lors de la fouille? L'état de notre documentation ne permet pas de conclure.
En conclusion, les prospections renvoient l'image d'un site principal, d'environ 1 ha, autour duquel se développent des pôles annexes. La présence d'éléments résidentiels dans la concentration principale invite à restituer un habitat confortable avec une partie résidentielle. La superficie de ce noyau principal, 1 ha, est comparable à celle de nombreuses villae de la région (Buffat 2004). L'hypothèse d'une villa est la plus probable.

\section{4 - L'ÉTABLISSEMENT DU BAS-EMPIRE: LES VESTIGES DE BÂTIMENTS AGRICOLES} (phase $\mathrm{C}, \mathrm{III}^{\mathrm{e}}-\mathrm{V}^{\mathrm{e}}$ siècle)

Toutes les informations convergent pour montrer que le site de Mayran connaît une forte activité tout au long du Bas-Empire. Les prospections montrent que l'occupation s'est déplacée par rapport au Haut-Empire. L'établissement alto-impérial a été abandonné pour faire place à un nouvel ensemble, installé $50 \mathrm{~m}$ au nord-est. Ce nouvel ensemble couvre une large surface, probablement supérieure à 2 ha, et dispose apparemment d'hypocaustes, si l'on en croit les débris présents dans certains secteurs. La découverte d'un important complexe vinicole, qui correspond (apparemment) à la pars fructuaria du domaine, illustre la vitalité de l'exploitation. Celui-ci sera utilisé de façon continue au moins jusqu'au $\mathrm{V}^{\mathrm{e}} \mathrm{s}$. Ces installations vinicoles sont malheureusement extrêmement dégradées par les travaux agricoles récents et par le creusement de silos à l'époque médiévale. Il n'en subsiste que les aménagements en creux, en l'occurrence des cuves et les fondations de murs. Ceci limite (drastiquement) la compréhension de la chaîne de production, du pressurage au

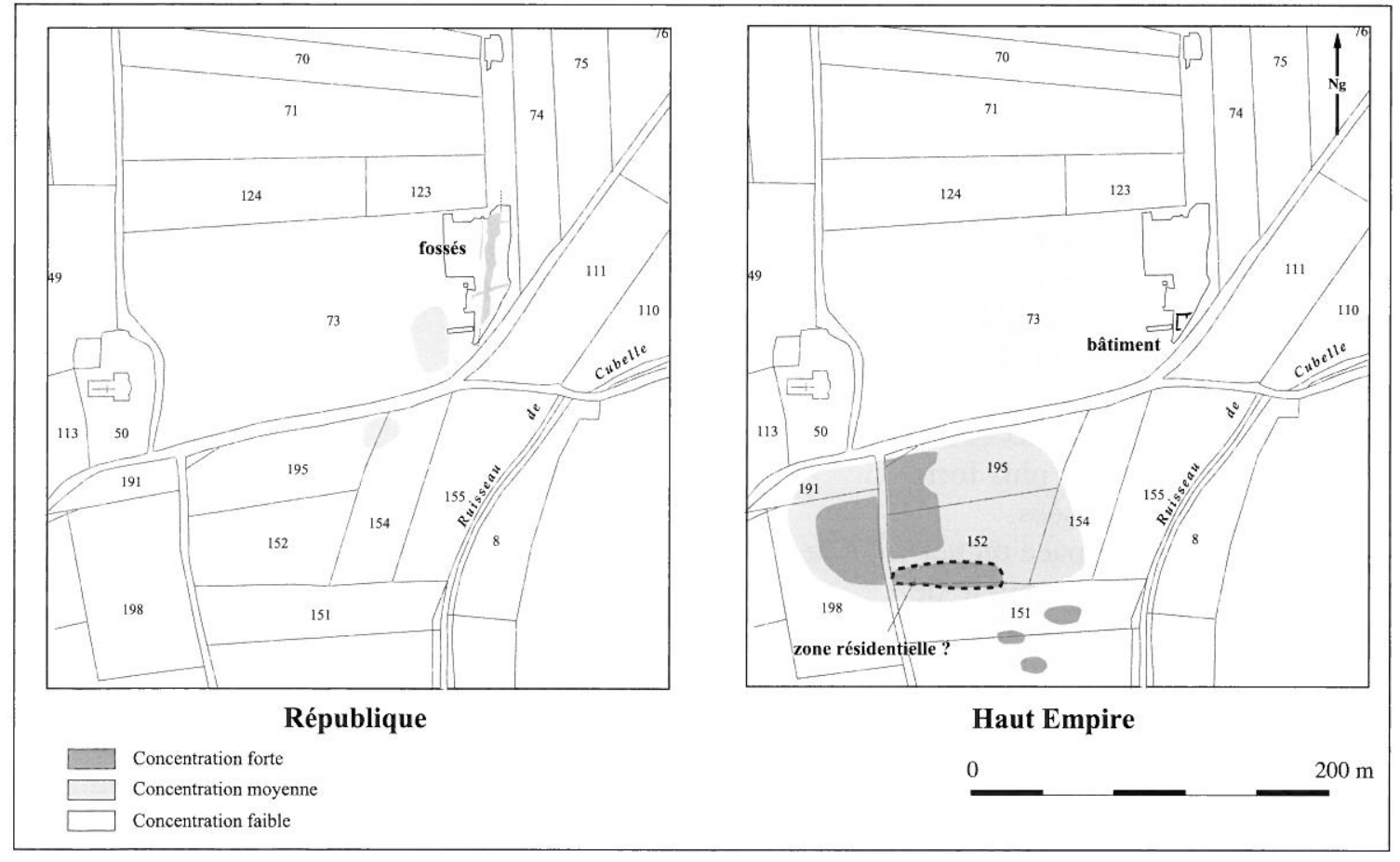

Fig. 21. L'évolution du site de Mayran entre la période républicaine et le Haut-Empire : concentrations de mobilier enregistrées lors des prospections de surface. 


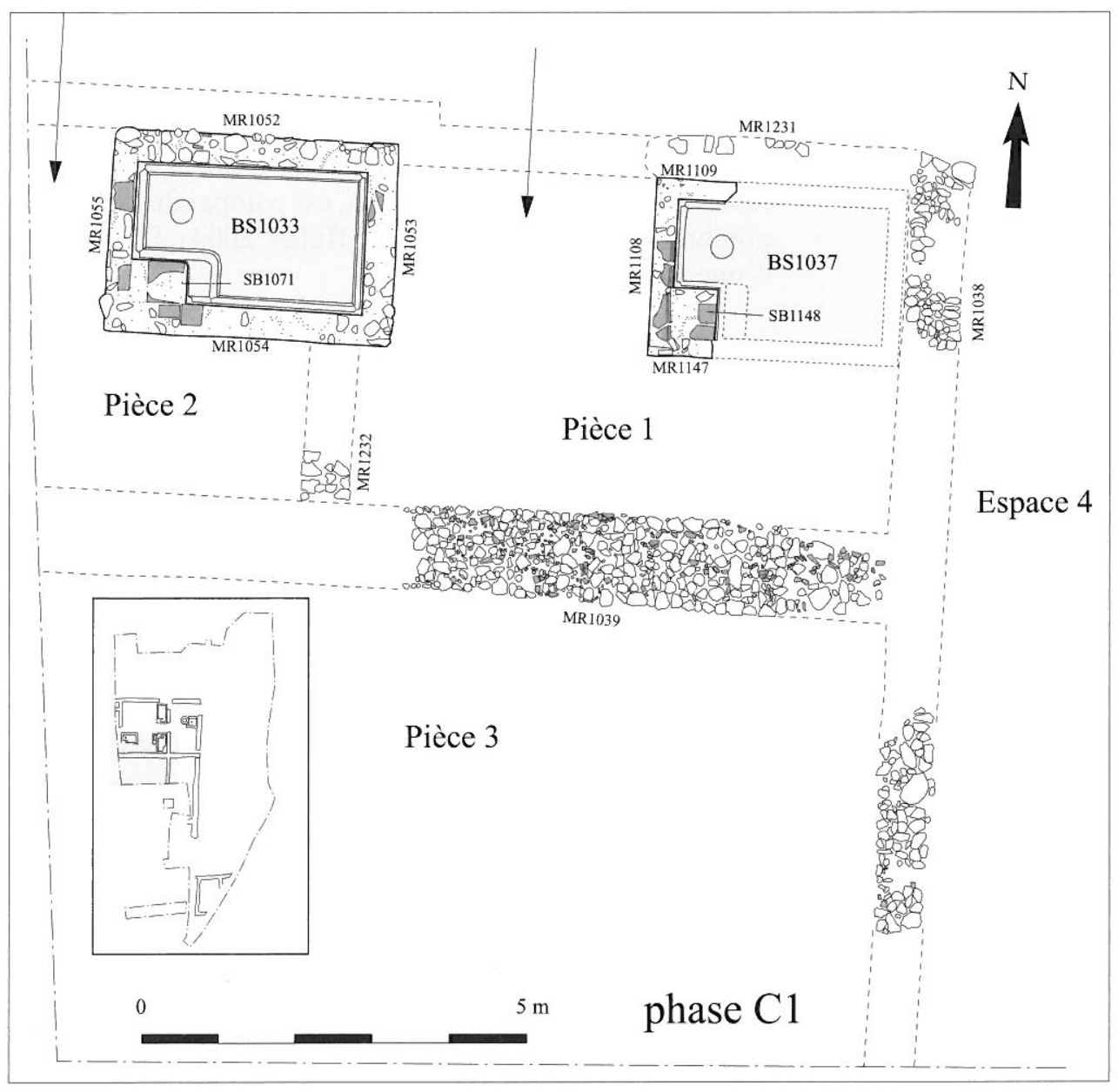

ci-contre

Fig. 22. Les aménagements vinicoles de la phase C1.

ci-dessous

Fig. 23. Coupe de la cuve 1033.

stockage. Les traces d'un vignoble sont en revanche présentes en marge de la villa. Elles nous renseignent - et nous interrogent - sur les modes de culture de la vigne au sein du domaine.

\subsection{Les premières cuves (phase $\mathrm{C} 1$ - $\mathrm{III}^{\mathrm{e}} \mathrm{s}$.?)}

Le premier état du complexe agricole occupe, dans l'emprise de la fouille, une surface d'environ $145 \mathrm{~m}^{2}$ (fig. 22). Les constructions utilisent un matériau singulier, un calcaire bleuté et alvéolé, provenant de bancs de tufs quaternaires situés à un kilomètre au sud-est du site (fig. 4). Ce bâtiment a souffert successivement des réaménagements postérieurs, des épierrements médiévaux et des labours modernes. La lecture des vestiges et, à plus forte raison, leur interprétation s'en trouvent pénalisées.

La pièce 1, située au nord-est, décrit un espace rectangulaire de 7 x 4,5 m (fig. 22) couvrant une superficie de $30 \mathrm{~m}^{2}$. Son angle nord-est est occupé par une cuve (1037), perturbée par des constructions postérieures. De direction est-ouest, cette cuve est large de 1,8 m (dans œuvre). Si l'on restitue sa limite orientale au contact du parement ouest du mur 1038, sa longueur atteint $2,7 \mathrm{~m}$. Ses murs $(1109,1108,1147)$ sont constitués de pierres calcaires et

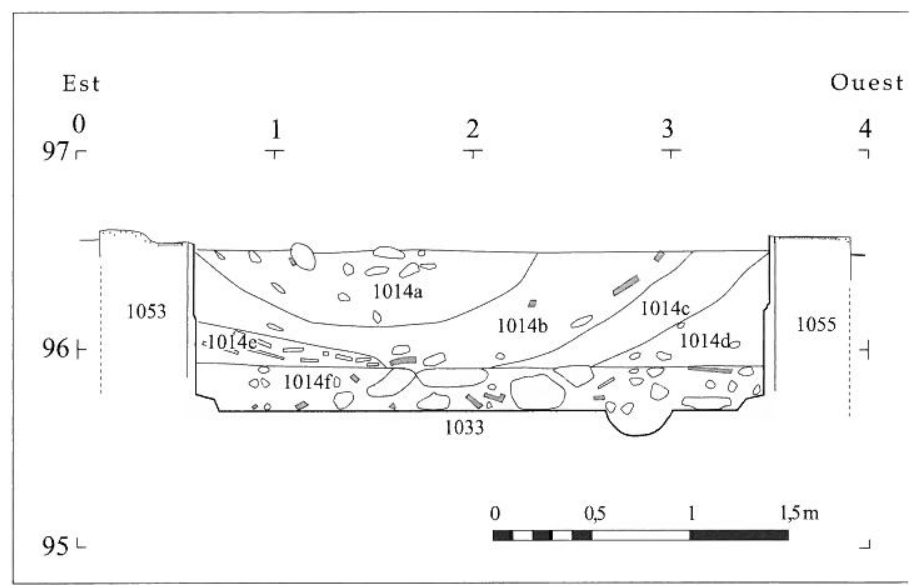

de tuiles assisées et liées au mortier. Ses parois internes sont enduites d'un béton hydraulique granuleux (éclats de tuiles inférieurs au centimètre). Chaque arête est renforcée par un boudin anguleux. L'escalier (1148), situé à l'angle sud-ouest, comporte deux marches d'une quarantaine de $\mathrm{cm}$ de hauteur. Autour de cette cuve, un espace libre, en forme de "L", correspond soit à une zone de circulation permettant d'accéder au bassin, soit à l'emplacement des 


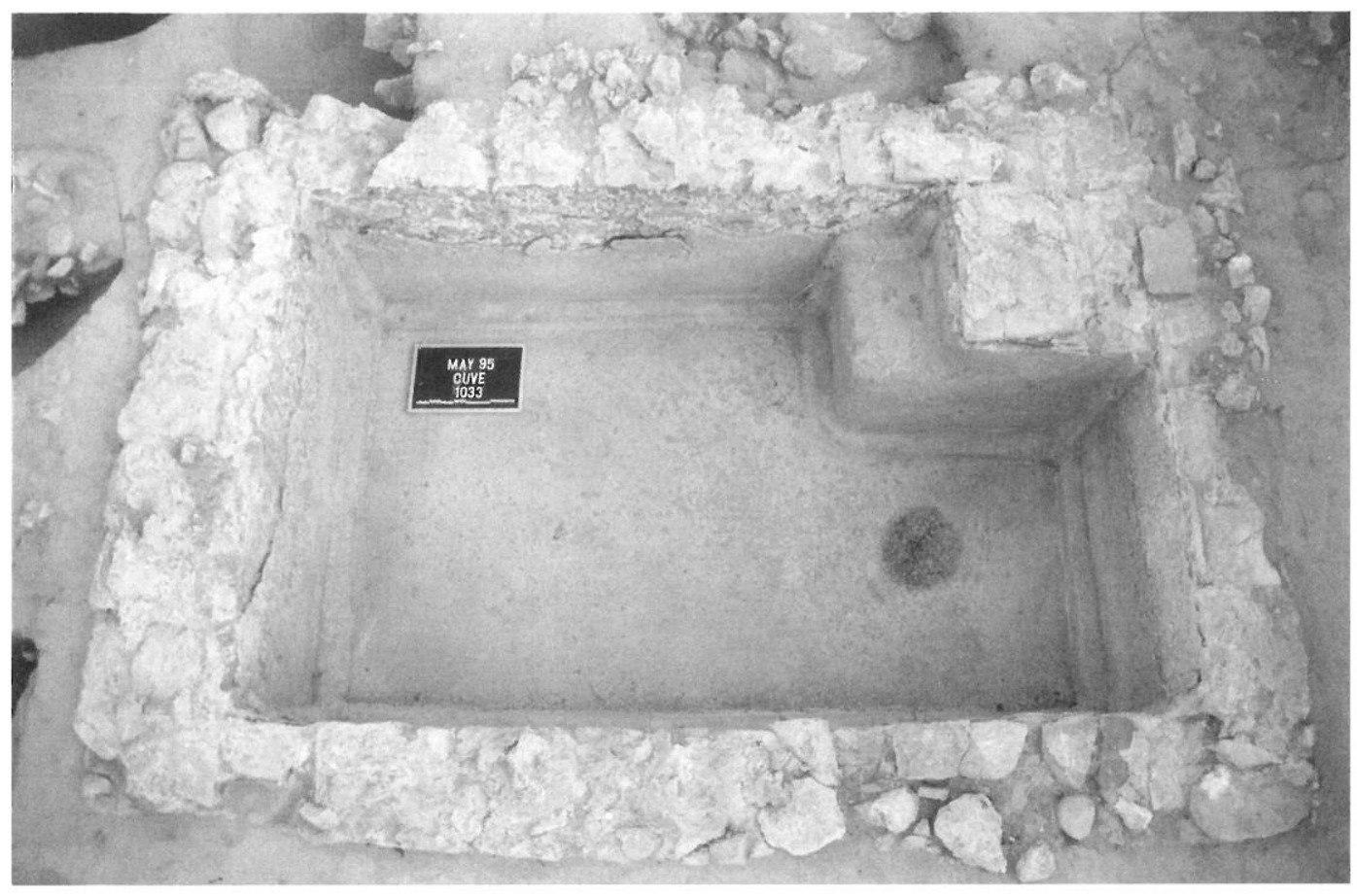

Fig. 24. La cuve 1033 vue du nord (cliché $\mathrm{H}$. Petitot).

installations de pressurage. L'entrée de la pièce peut être restituée au nord-ouest.

La pièce 2, implantée au nord-ouest, offre un plan plus lacunaire (fig. 22). Elle comporte à l'est une cuve. De direction est-ouest, celle-ci mesure $3,0 \mathrm{~m}$ de long et $1,8 \mathrm{~m}$ de large (dimensions dans œuvre). Conservée sur une profondeur de $0,9 \mathrm{~m}$, elle présente un fond plat creusé vers l'est d'une alvéole d'un diamètre de $0,3 \mathrm{~m}$. Ses murs (1052, $1053,1054,1055)$ sont constitués de pierres calcaires et de tuiles assisées et liées au mortier. Ses parois internes sont intégralement recouvertes d'un béton hydraulique à texture inégale: l'enduit mural contient des fragments de tuiles très fins, tandis que la chape de fond est constituée d'agrégats plus grossiers. Chaque arête interne est consolidée par un boudin anguleux. Un escalier situé à l'angle sud-ouest, permettait de descendre dans la cuve grâce à deux marches d'une cinquantaine de $\mathrm{cm}$ de hauteur (fig. 24). Les murs 1039 et 1232 constituent les limites méridionale et orientale de la pièce où se trouve cette cuve. Son mur nord, totalement évacué, semble s'être situé dans l'alignement du mur 1231 , légèrement en contre-haut de celui-ci. L'organisation interne de la pièce 2 est pratiquement identique à celle de la pièce 1 : entrée probable au nord-ouest permettant un accès immédiat à la cuve (1033), zone de circulation et/ou d'activité dans un espace en "L". Ces analogies n'impliquent cependant pas une stricte contemporanéité des deux espaces. Un examen attentif révèle des différences dans la mise en œuvre de chacune des cuves: dimensions, texture des bétons hydrauliques, épaisseur des murs, qualité de mortier. Par ailleurs, la cuve 1033 semble oblitérer l'angle nord-ouest de la pièce 1 , et par voie de conséquence être mise en place un peu plus tard.
L'espace 3 occupe la partie sud du complexe. Il couvre, dans l'emprise de la fouille, une surface d'environ $60 \mathrm{~m}^{2}$. Il n'a livré aucun indice susceptible d'indiquer sa fonction. On pense à une zone de stockage pour des tonneaux, mais rien ne permet d'en être sûr. L'espace 4 correspond à la périphérie du bâtiment. Zone de proche campagne ou jardin, elle ne livre aucun aménagement particulier.

\section{Datation des constructions}

La datation de ces constructions est délicate à préciser faute de mobilier. Parmi les quelques tessons recueillis dans la chape du bassin 1037, on ne compte qu'une panse d'amphore africaine associée à des éléments du HautEmpire (amphore gauloise, claire récente). Ces éléments fournissent un terminus post quem large, calé sur le $\mathrm{III}^{\mathrm{e}} \mathrm{s}$.

\section{INVENTAIRE DU MOBILIER}

\section{Chape du bassin 1037}

1037 (200/300) phase C1- claire récente: 4 fr. (1 fond), kaol. réductrice : 2 fr., amphore gauloise : 2 fr., amphore hispanique: $1 \mathrm{fr}$., amphore africaine : $1 \mathrm{fr}$. Total céramiques: $11 \mathrm{fr}$.

\subsection{Agrandissements et mise en place de nouvelles cuves (phase $\mathbf{C 2}, \mathbf{I V}^{\mathbf{e}}$ s.) (fig. 25 à 28)}

La phase qui succède à la construction du premier complexe est marquée par une extension des aménagements agricoles vers le nord et par la construction de deux nouvelles cuves (fig. 25). Les nouvelles constructions respectent la trame architecturale de la phase précédente et conservent une orientation strictement identique. Les maté- 


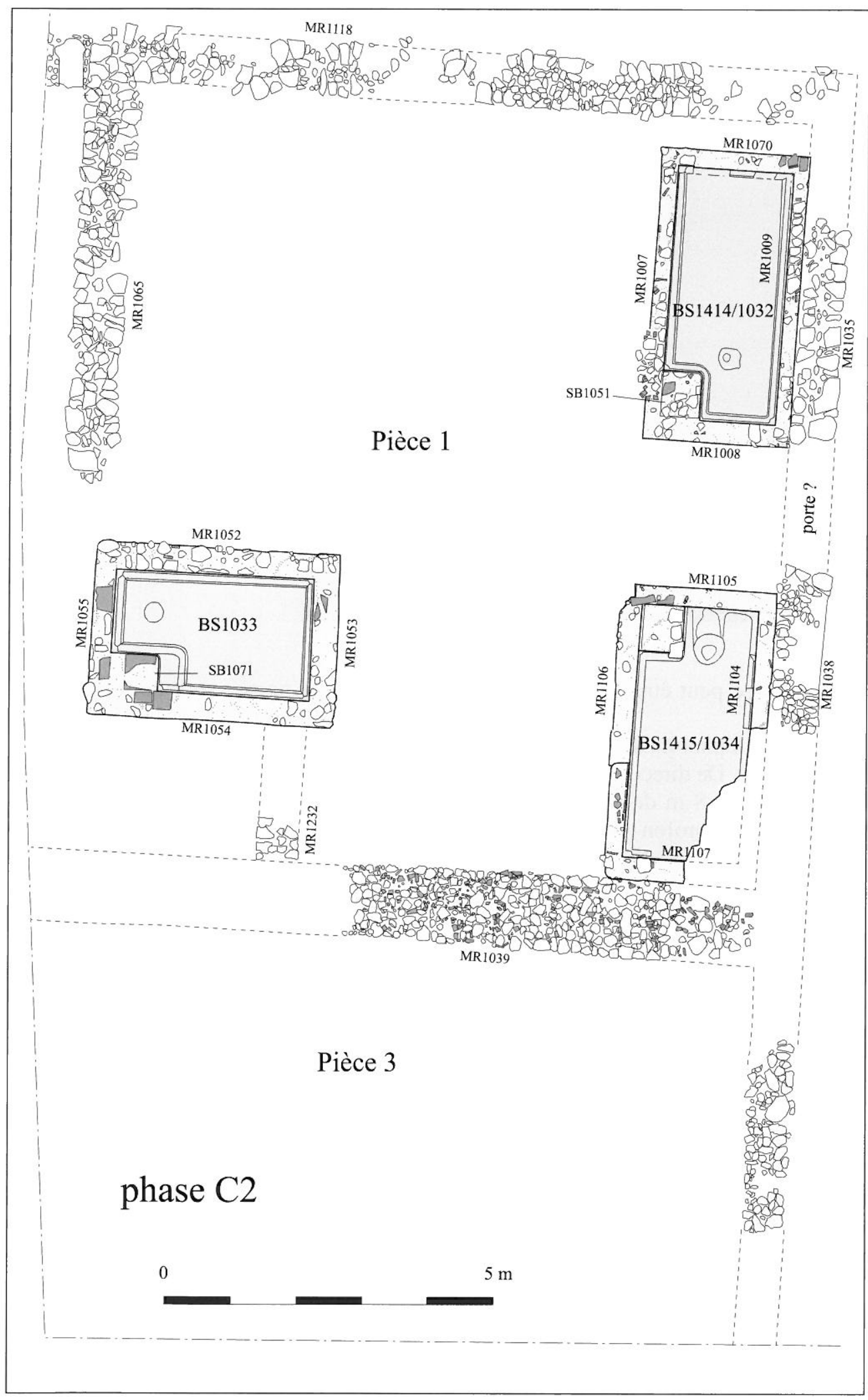

Fig. 25. Plan et coupes des aménagements de la phase C2.

riaux mis en œuvre durant cette nouvelle campagne de travaux se différencient nettement de ceux de la phase antérieure. À l'utilisation des tufs quaternaires bleutés, succède celle du calcaire froid (urgonien) et du galet. L'espace dévolu aux cuves, étriqué jusqu'alors, devient plus large.
Certains murs de la phase précédente sont éradiqués pour faire place à une salle unique (pièce 1: $120 \mathrm{~m}^{2}$ ). La cuve 1037 est abandonnée et le mur 1231 intégralement évacué. Ne sont conservées que les limites méridionale et orientale du premier cellier (murs 1038 et 1039). Deux 
ci-contre

Fig. 26. Le bassin 1415/1034 recoupant une cuve plus ancienne (cliché H. Petitot).

ci-dessous

Fig. 27. Coupe des bassins $1414 / 1032$ et $1415 / 1034$ (dessin H. Petitot).
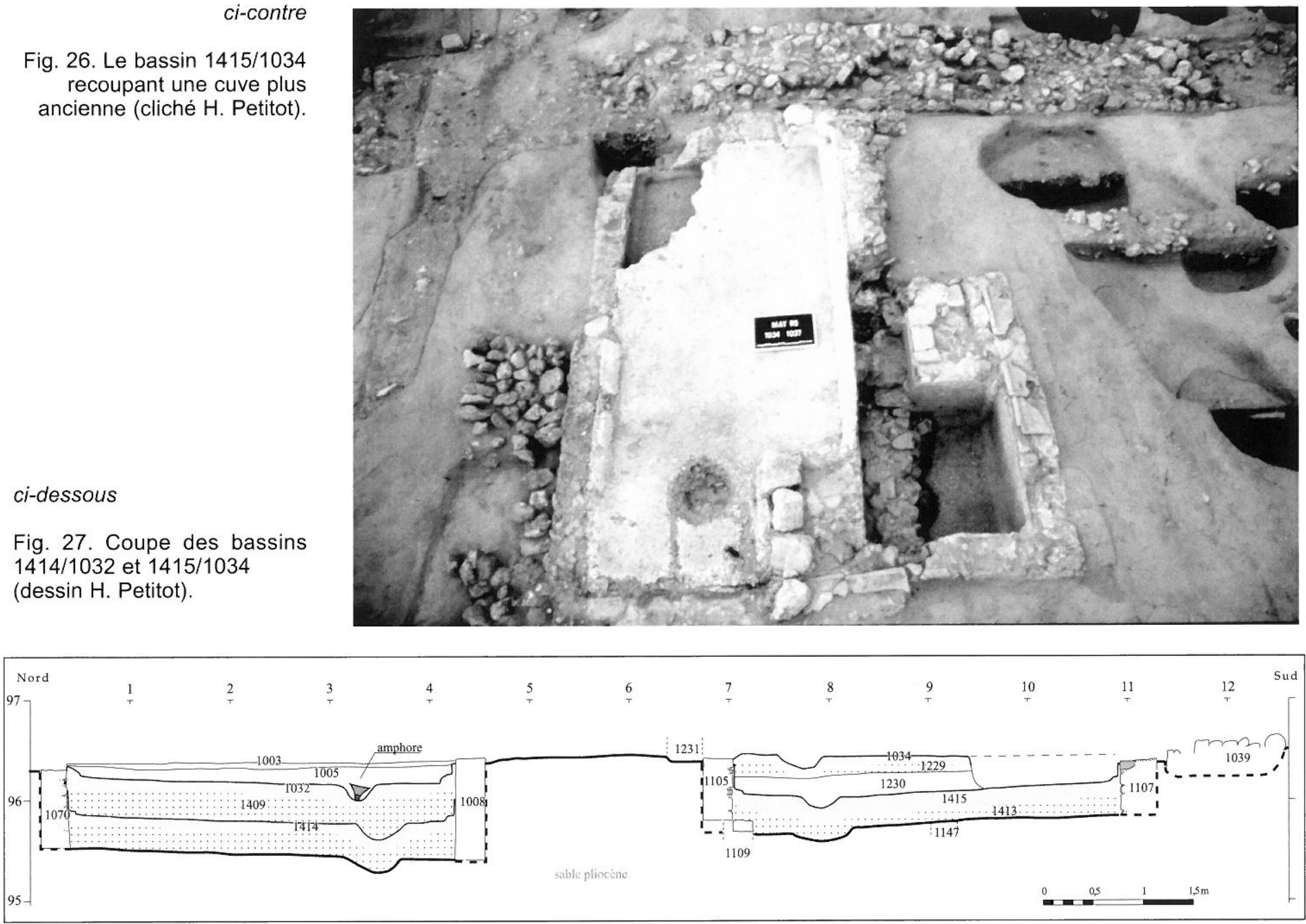

cuves (1414/1032, et 1415/1034) prennent place dans la partie est de la nouvelle salle. De dimensions sensiblement identiques, elles marquent une restructuration de l'espace. Aux cuves ramassées et profondes de la phase antérieure succèdent ces bassins longilignes. Ces cuves ont connu deux états successifs marqués par deux niveaux de chapes. Les plus anciennes chapes (1414 et 1415) présentent un revêtement d'opus signinum granuleux où dominent les gros éclats de tuiles. Les enduits de parois incluent des agrégats beaucoup plus fins. Les cuves comportent un emmarchement double.

Dans le second état, le fond de ces cuves est rehaussé d'une quarantaine de $\mathrm{cm}$ par la mise en place de nouvelles chapes (1032 et 1034, fig. 25). L'une d'elles (1032) comporte un aménagement intéressant. Dans la cupule, un fond d'amphore a été retrouvé en place. Il s'agit d'un fond d'amphore africaine (pâte brique feuilletée, engobe blanc épais), comportant un pied court annelé, attribuable à la forme Keay LVII ou Keay LXII (fig. 28). Ce dernier semble avoir été utilisé pour faciliter le nettoyage et l'évacuation des résidus de décantation déposés au fond du bassin. L'entrée de la pièce aux cuves peut être restituée à l'est, entre les murs 1035 et 1038 . Une autre pourrait prendre place, vers l'ouest, au sud du mur 1065. Durant cette période, un doute subsiste sur le devenir de la cuve antérieure 1033. Continue-t-elle de fonctionner ou est-elle remblayée? Son comblement, remanié à la fin du Moyen Âge (fig. 23), ne livre aucun élément de réponse.

\section{Datation}

Les éléments permettant de caler chronologiquement cette phase sont une nouvelle fois peu nombreux. Des fragments d'amphore africaine recueillis dans les constructions (fondations de murs, radiers de bassin) ne font que confirmer la datation tardive de ce complexe. Les rechapages des deux cuves ne sont pas mieux datés. Les niveaux de radier ne livrent comme éléments caractéristiques que quelques panses d'amphore africaine et d'amphore de Bétique tardive (Dr. 23 à pâte calcaire). On note cependant dans ces maigres lots l'absence de céramique à pisolithes, qui tendrait à situer les constructions et remaniements avant la fin du $\mathrm{IV}^{\mathrm{e}} \mathrm{s}$. Le fond d'amphore africaine trouvé dans la cuve 1032 indique un abandon tardif, probablement la fin du $\mathrm{V}^{\mathrm{e}}$ ou le $\mathrm{VI}^{\mathrm{e}} \mathrm{s}$. 


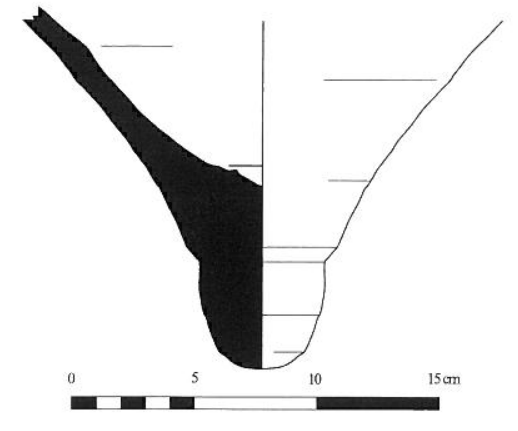

Fig. 28. Fond d'amphore africaine utilisé pour évacuer les bourbes de décantation dans la cuve 1032 .

INVENTAIRE DU MOBILIER

Mur 1065 (275/400) phase C2a - oxydante micacée: 1 fr., amphore africaine: $2 \mathrm{fr}$. Total céramiques: $3 \mathrm{fr}$

Comblement de la cuve 1037

1097 (275/400) phase C2a - amphore africaine : $1 \mathrm{fr}$.

Radier de la cuve 1415

1413 (250/400) phase C2a - amphore gauloise: 1 fr., amphore africaine: $1 \mathrm{fr}$. Total céramiques: $2 \mathrm{fr}$.

Rechapage des cuves (phase $C 2 b$ )

- Remblai de construction de la cuve 1034 :

1229 (300/425) phase C2b - claire B : 1 fr., amphore gauloise :

1 fr., amphore de Bétique : 2 fr., amphore africaine : 5 fr. Total céramiques: $9 \mathrm{fr}$.

- Radier de la cuve 1032 :

$1409(300 / 425)$ phase C2b - amphore africaine : $3 \mathrm{fr}$.

\subsection{Contenance des cuves: estimations et comparaisons}

Il est difficile d'évaluer la capacité des cuves étant donné l'état d'arasement des vestiges. Néanmoins, si l'on estime que l'épaisseur de la couche archéologique évacuée se situe entre 0,2 et $0,5 \mathrm{~m}$, il est possible d'avancer les minima suivants: 32001 . pour la cuve 1032, 51361 . pour le premier état de la cuve 1034,19261 . pour le deuxième état, 57511 . pour la cuve 1033 et 52351 . pour la cuve 1037. Ces estimations indiquent a priori une plus forte capacité de stockage des cuves les plus anciennes (1033, 1037), mais, les cuves récentes (1032 et 1034) ayant été davantage exposées aux travaux agricoles (du fait de leur position stratigraphique plus haute), l'observation n'a qu'une valeur relative.

La contenance des cuves de Mayran se place dans la moyenne régionale: au Mollard (Drôme), 40001. (Dechandol et al. 1983), à Pardigon 1/3 (Var), 50001. (Brun, Congès 1988, 48), au Grand Loou (Var), 3000 et 5000 1. (Brun 1986, 192-193), à Gignac-la-Nerthe (Bouches-du-Rhône), 2912 et 3337 1. (Gateau 1993), à
Aix-en-Provence (Bouches-du-Rhône), 3000 1. (Nin 1987, 217). Le volume de celle découverte sur le site de la Combe de Fignols (Hérault), est par contre beaucoup plus important (8500 1., Olive 1993). Il s'agit néanmoins ici de la seule cuve dont cet établissement soit équipé.

\subsection{Pressurage, foulage : quelques hypothèses sur l'installation de Mayran}

Il convient de s'interroger maintenant sur la localisation des installations de pressurage/foulage en relation avec les cuves. L'exercice n'est pas facile compte tenu de l'arasement des vestiges. Les pressoirs et fouloirs sont généralement des aménagements peu conservés, car ils surplombaient les autres installations dans les chais. Ils sont surélevés «comme la tribune d'une basilique» selon les dires de Palladius (I, 18). On ne peut donc guère avancer de certitudes. Quelques comparaisons avec des installations mieux conservées peuvent cependant nous éclairer.

Rappelons d'abord que les cuves dégagées à Mayran présentent des caractéristiques que l'on retrouve tout au long du fonctionnement du chai. Outre la présence systématique d'un emmarchement, elles fonctionnent toujours par paires. Cette disposition, à cuve double, se rencontre dans d'autres installations romaines. Ainsi à Brauneberg, dans la vallée de la Moselle, K.-J. Gilles a dégagé une vaste installation vinicole que l'on peut comparer à celle de Mayran. Deux complexes de pressurage du BasEmpire ont été exhumés à chacune des extrémités d'un long bâtiment de $36 \times 7,5 \mathrm{~m}$ (fig. 30). L'installation dégagée à l'est comporte deux cuves surmontées d'un fouloir et d'un pressoir (Gilles 1990, 33-39). Les structures de pressurage occupent un espace situé entre les cuves et les murs gouttereaux du bâtiment. On y accédait par un escalier situé entre les deux cuves. C'est un schéma que l'on retrouve aussi sur la villa de Saint-Martin à Taradeau (Var) $(C A G$ 83/2, 765-770).

Ces exemples peuvent nous aider à restituer les aménagements de l'état le plus ancien de Mayran (phase C1, fig. 29). Il serait, à partir de ces comparaisons, logique de situer les installations de pressurage au sud des cuves, dans un espace longiligne, large de $2 \mathrm{~m}$, dans lequel aucun aménagement n'a été repéré. L'arasement très prononcé des vestiges rend difficile une restitution. N'y avait-il que des fouloirs? Il est difficile de se prononcer. Cependant, le mur dégagé au sud des cuves présente des fondations très puissantes, en particulier une largeur supérieure à l'habituel $(1,1 \mathrm{~m})$. Il n'est pas impossible que ce mur ait servi d'ancrage à un ou des pressoirs. Un prelum à levier pouvait par exemple être ancré dans ce mur et être manié au nord des cuves, selon un système proche de celui observé dans le cellier oriental de Brauneberg (Gilles 1991, 21-26, restitution proposée p. 25 , Abb. 5). Il est vrai cependant qu'aucune fosse de réception de contrepoids n'a été repérée, mais celle-ci a peut-être totalement disparu. 


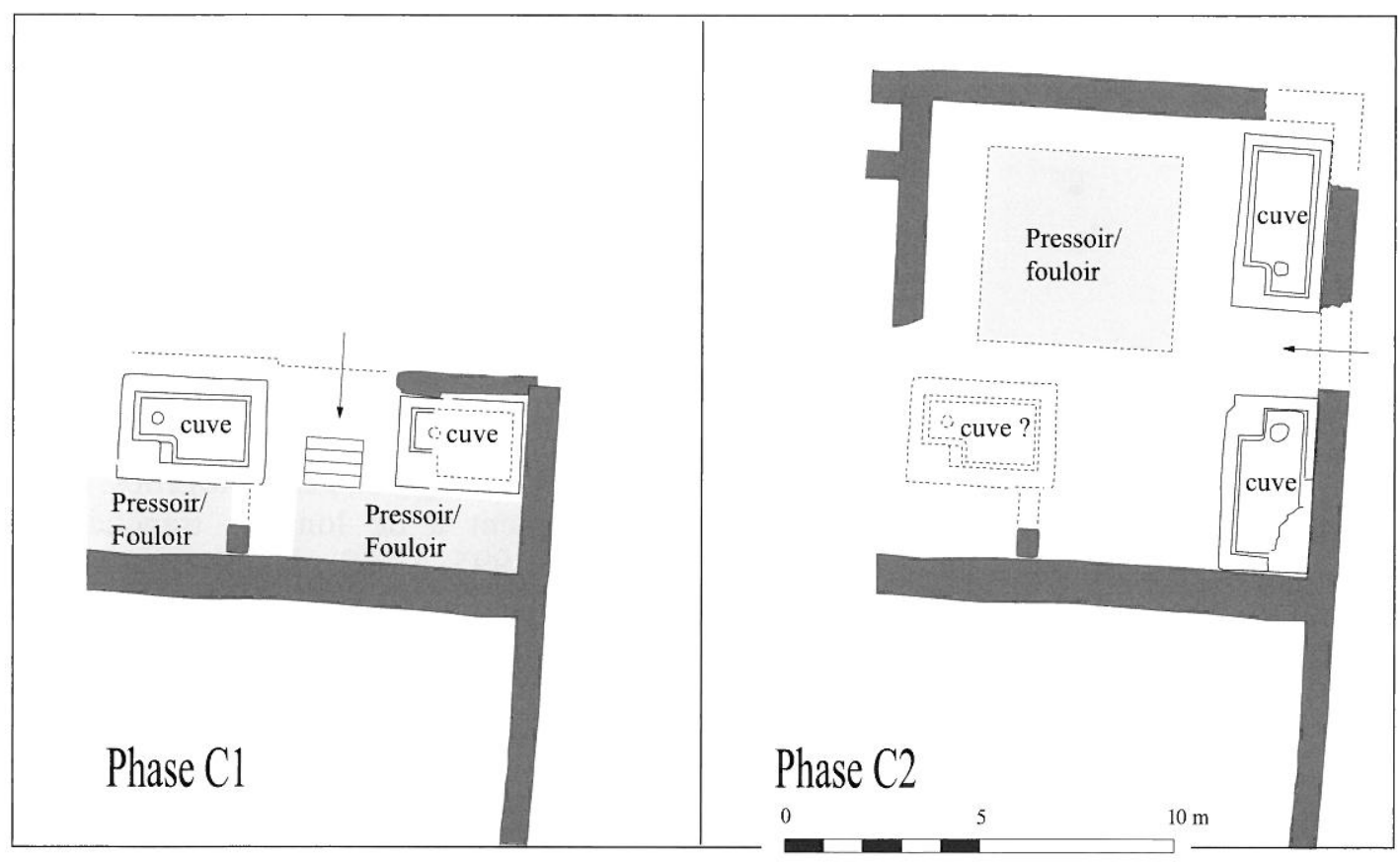

ci-contre

Fig. 29. Proposition de localisation des pressoirs et /ou des fouloirs du chai de Mayran.

\section{ci-dessous}

Fig. 30. L'installation de Brauneberg/Unter Mötschert en Allemagne (d'après Gilles 1991, 22, abb. 2).

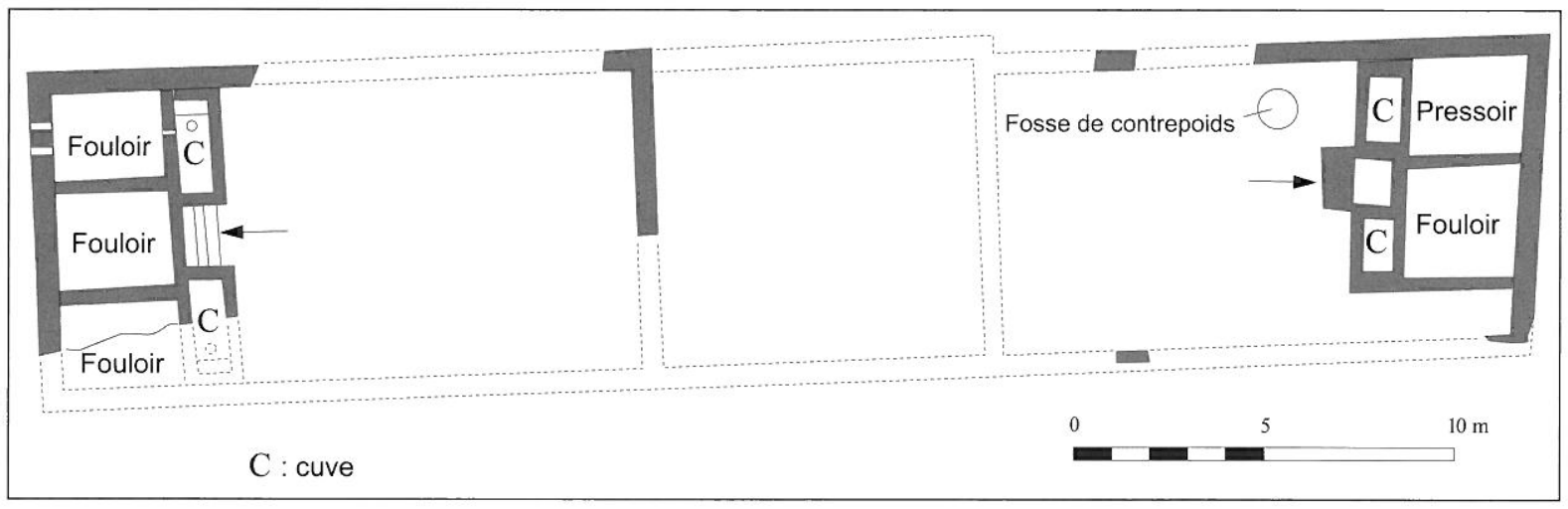

Pour le second état (phase C2, fig. 29), les hypothèses sont plus difficiles à émettre. L'absence de silos médiévaux dans une zone d'environ $25 \mathrm{~m}^{2}$ (au nord-est) constitue un indice auquel on est tenté de donner du sens. Leur creusement a probablement été empêché ou limité par une importante construction, peut-être une plateforme de pressurage ou de foulage. L'hypothèse est possible, sachant que les vestiges romains sont restés visibles longtemps: les cuves n'ont effectivement pas été recoupées par des silos. La cuve 1033 a de surcroît livré un comblement daté du $X I V^{e}-X V^{e} s$. qui indique qu'elle a été déblayée et peutêtre réutilisée à cette période.

Quelles que que soient les hypothèses retenues pour l'emplacement des installations de pressurage, l'existence de cuves fonctionnant par paires constitue le fait la plus marquant. Comment peut-on interpréter ce dispositif ? La première hypothèse est celle de cuves différenciées recueillant pour l'une les jus de presse, pour l'autre ceux du foulage. On n'exclura pas non plus une séparation des jus des deux pressées successives, comme le décrit Varron
(I, 54), afin de recueillir à part le jus de la seconde presse, tiré des rafles, réputé avoir un "un goût de fer".

\subsection{Un vignoble en bordure de la villa (fig. 31 à 33)}

Parallèlement à la découverte des installations du cellier, la production vinicole est documentée par la découverte, à l'extérieur du bâtiment, de petites fosses de formes oblongues, interprétées comme des traces de plantations de vigne.

Ces fosses se caractérisent par un comblement particulier, constitué exclusivement de galets. Les fosses sont, pour la plupart, de forme allongée et sont orientées selon un axe est-ouest. Leur longueur est comprise entre 0,6 et $1 \mathrm{~m}$, leur largeur se situe entre 0,3 et $0,6 \mathrm{~m}$. Leur profondeur varie entre 0,1 et $0,35 \mathrm{~m}$ (fig. 33). Les plus nombreuses se trouvent immédiatement à l'est des bâtiments agricoles. Elles ne dessinent pas d'alignements nets.

Ces fosses ont livré peu de mobilier. L'une d'elle a fourni cependant un bord d'urne KAOL A18 en céramique 


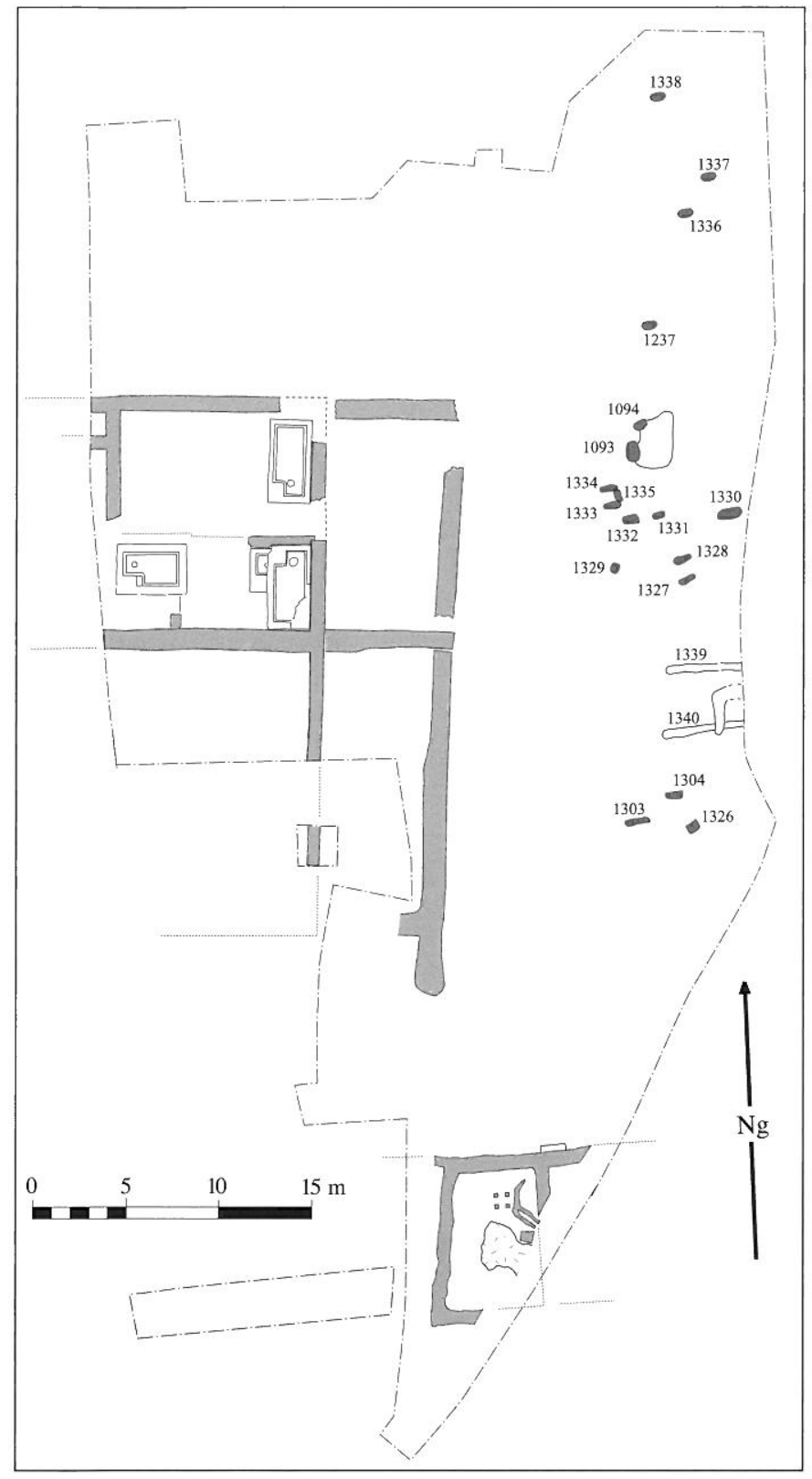

Fig. 31. Localisation des fosses de plantation antiques repérées sur le chantier de Mayran.

kaolinitique oxydante. Pour ces productions oxydantes, cette forme est connue régionalement dans des contextes de la seconde moitié du $\mathrm{IV}^{\mathrm{e}} \mathrm{s}$. et la première moitié du $\mathrm{V}^{\mathrm{e}}$ s. (Meffre, Raynaud in Py 1993, 490; Buffat, Petitot 1996, 93, fig. 6, n¹8). Elle disparaît sur la villa de La Gramière au début du $\mathrm{V}^{\mathrm{e}} \mathrm{s}$. (étude inédite, L. Buffat).

Par leur module et leur morphologie, ces fosses s'apparentent à celles des plantations viticoles, dont les découvertes se sont multipliées ces dernières années dans le sud de la France. Les plus anciennes que l'on connaisse se situent à Marseille sur le site de Saint-Jean du Désert.

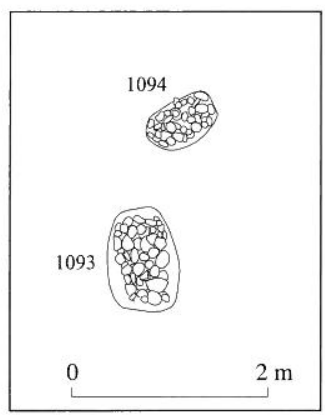

Fig. 32. Relevé de deux fosses de plantation avec leur comblement de galets.

Datées de l'âge du Fer, il s'agit de petites logettes, installées perpendiculairement à de longues tranchées de défonçage (Boissinot 1995, 33-39), interprétées comme des fosses de marcottage à usage viticole (Ibid., 37). Les exemples romains sont nombreux. On renverra pour ce type de traces aux synthèses sur Nîmes (Monteil et al. 1998), sur le Languedoc central et oriental (Vidal 2000) ou encore le Midi (Boissinot 2001, 47-63). On citera l'exemple proche dégagé au Mas Carbonnel, à Nîmes, et daté du Haut-Empire (Petitot, Vidal 1993). Sur plusieurs centaines de mètres carrés, de nombreuses fosses, identiques à celles de Mayran, marquent l'emplacement de champs antiques. D'autres exemplaires, datés de l'Antiquité tardive sont connus sur le site de Dassargues à Lunel (Favory et al. 1993, 155, 160). Longues d'un mètre et larges de vingt centimètres, les fosses sont implantées dans des parcelles délimitées par des fossés. Elles sont alignées à intervalles réguliers et forment des rangs parallèles espacés de 1,2 à 1,3 m. L'encroûtement calcaire (le "taparas") paraît ici avoir été brisé à la pioche pour faciliter la pénétration des racines.

Les exemples de plantations viticoles, que l'on pourrait multiplier, révèlent l'ancienneté et la continuité de cette pratique culturale. Bien qu'irrégulières dans leur morphologie, les fosses dégagées à Mayran s'apparentent clairement à ces exemples. On constate cependant que contrairement à des cas comme Nîmes/Mas Carbonnel ou Dassargues, les fosses ne se répartissent pas selon un schéma strict et ne sont présentes que de façon irrégulière. Il apparaît donc que ce vignoble supposé ne nous est parvenu que sous une forme lacunaire. Il est probable que les fosses observées correspondent aux traces d'une intervention postérieure sur le vignoble. Les traces de la plantation initiale nous ont vraisemblablement échappé, la terre extraite lors du creusement des fosses ayant été remise dans le comblement.

Reste que la présence exclusive de galets dans le remplissage des fosses est difficilement explicable. S'agit-il d'aménagements drainants? Magon et à sa suite Columelle (III, 15, 4-5) conseillent de placer quelques pierres dans les fosses, dans le but d'écarter l'eau des racines pendant l'hiver, et de protéger ces dernières de la chaleur pendant l'été. Les fosses dégagées à Mayran témoignent-elles de cette pratique? C'est peu probable, le substrat sableux présent 


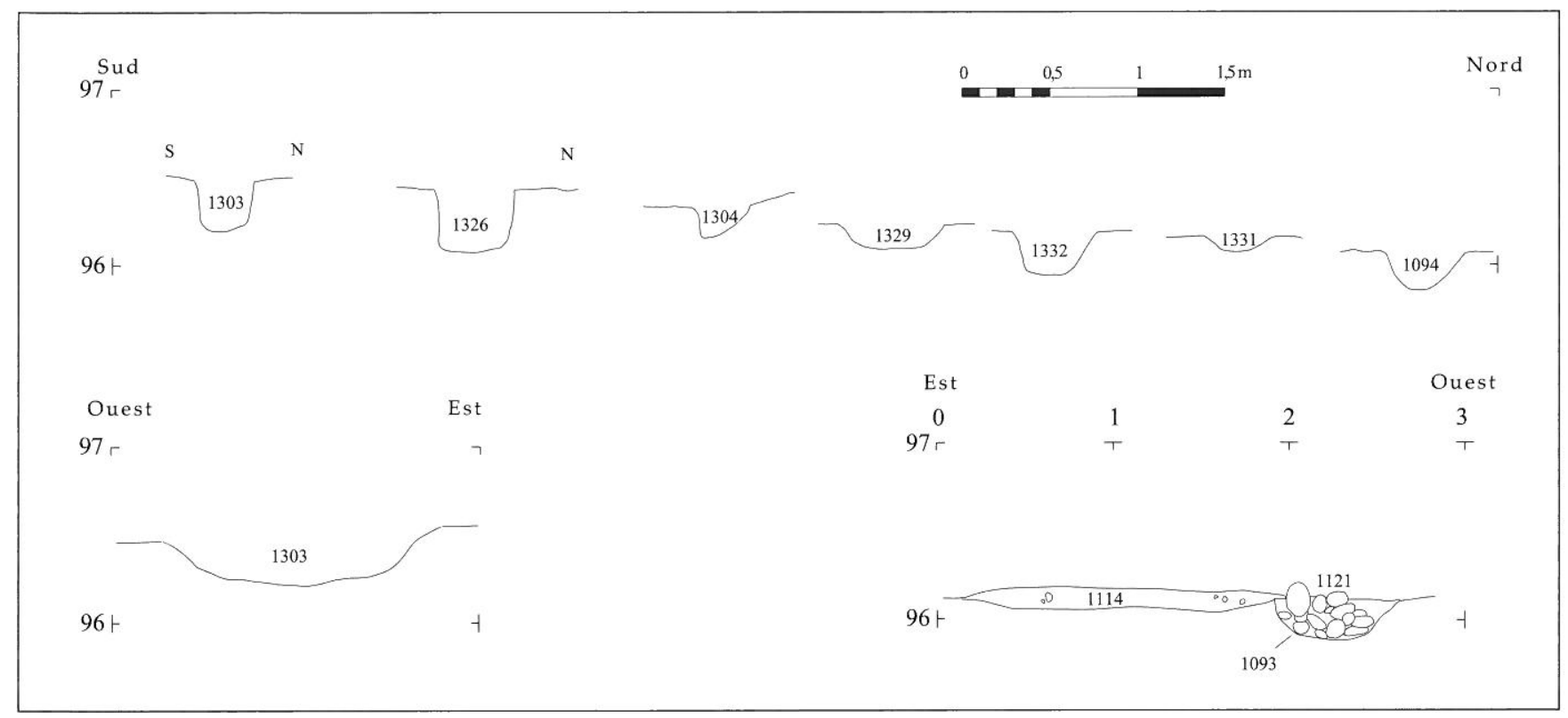

Fig. 33. Profil des fosses de plantation fouillées.

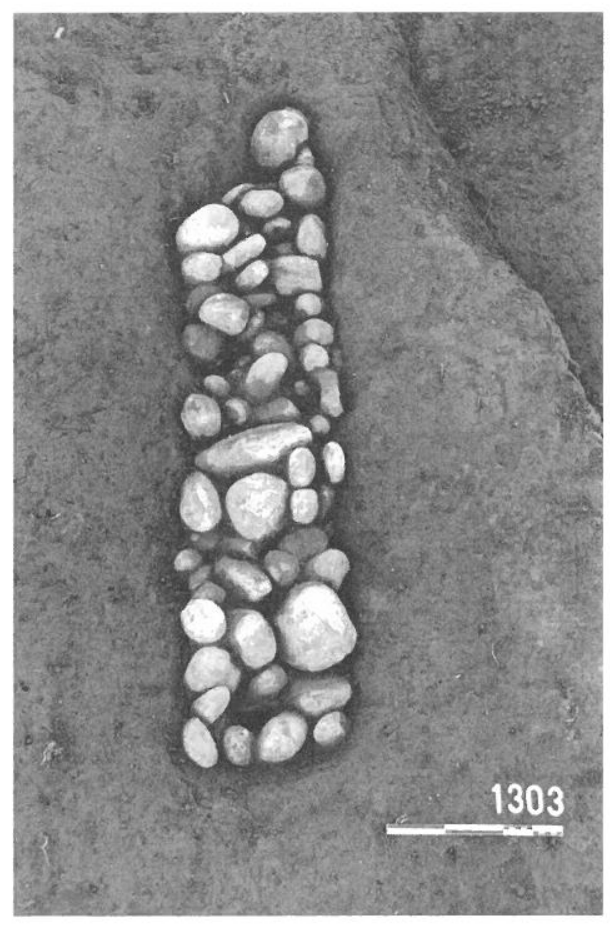

Fig. 34. Vue de l'une de l'une des fosses de plantation (cliché L. Buffat).

sur le site étant naturellement bien drainé. Il paraît plus vraisemblable que les agriculteurs aient ici tenté de reconstituer ponctuellement les conditions des terrasses würmiennes. Sur ces terrasses, les galets abaissent le rendement viticole, mais augmentent le degré alcoolique du raisin, assurant ainsi un vin de qualité. La vigne s'accommode très bien de la présence des galets: calorifuges, ils tiennent le cep à bonne température et permettent d'augmenter le taux de sucre des raisins. Il faut d'ailleurs rappeler que les qualités des sols pierreux pour la vigne n'avaient pas échappé aux agronomes antiques (sur cette question, voir Billiard 1913, 295). Si l'on peut donc sérieusement envisager cette hypothèse, il reste plus difficile de déterminer si ces fosses particulières ont été aménagées lors de la mise en place du vignoble, dans le cadre d'un "panachage" de la plantation, ou si elles relèvent d'une intervention postérieure. L'irrégularité des modules et les différences d'orientation invitent plutôt à retenir la seconde hypothèse. Dans ce cadre, il pourrait s'agir des traces d'une replantation ou bien celle d'un déchaussage de quelques souches au pied desquelles on a disposé des galets.

$\mathrm{Au}$ sud de cette zone agraire, le dispositif de fosses est accompagné de deux tranchées oblongues larges d'une quarantaine de centimètres $(1339,1340$, fig. 31$)$. Ces tranchées sont distantes de $2,9 \mathrm{~m}$ soit 10 pieds. Leur comblement se distingue nettement de celui des fosses puisqu'il est composé d'une terre sableuse ocre dépourvue d'inclusions. Ces saignées sont vraisemblablement en relation avec une préparation du terrain préalable. Il a effectivement été montré qu'il pouvait s'agir de tranchées de défoncement en relation avec la plantation viticole (Vidal 1998, 43-47).

\subsection{Image générale du site au Bas-Empire: les données de la prospection}

Sans les prospections de surface, on serait bien en peine d'interpréter le cadre général dans lequel s'insèrent les installations du Bas-Empire dégagées sur ce site. 


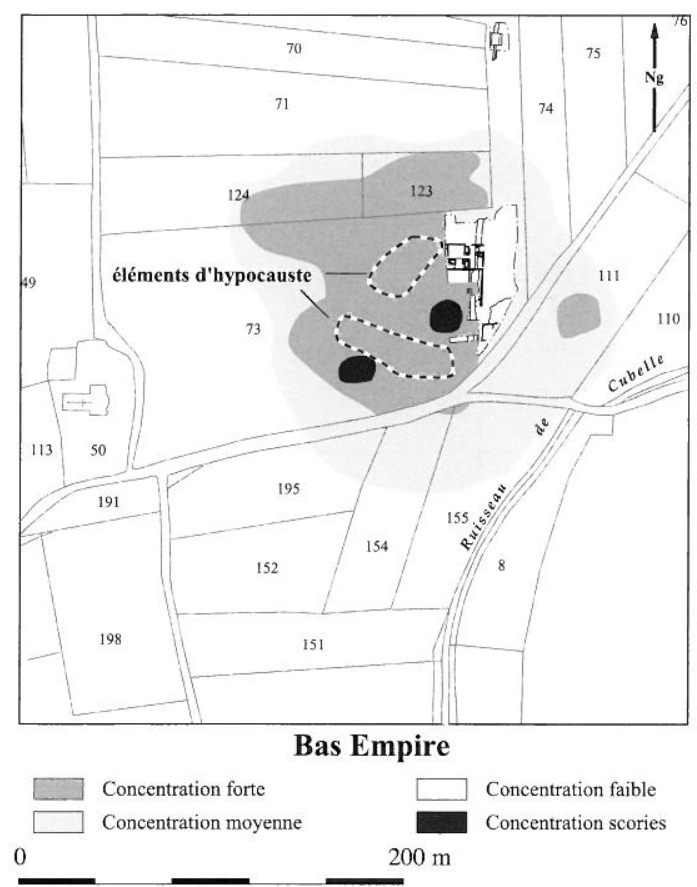

Fig. 35. Concentrations de mobilier du Bas-Empire repérées en prospection de surface.

Heureusement, les prospections apportent ici une image claire: les constructions fouillées se développent à l'est d'un ensemble tardif beaucoup plus vaste (fig. 35). Il apparaît clairement qu'un déplacement de l'occupation s'est produit depuis le Haut-Empire. Le nouvel établissement s'installe $100 \mathrm{~m}$ au nord-est de l'ancienne installation, totalement délaissée semble-t-il. S'il est clair que l'habitat a changé d'emplacement, il reste en revanche plus difficile de cerner les modalités et la chronologie du phénomène. S'agit-il d'un déplacement brutal, ou progressif? Ce déplacement se produit-il dans la seconde moitié du $\mathrm{II}^{\mathrm{e}} \mathrm{s}$. ou au $\mathrm{III}^{\mathrm{e}} \mathrm{s}$. ? Il est fort difficile de se prononcer à partir des seules données de la prospection.

La plus forte concentration d'éléments du Bas-Empire (amphore africaine, céramique à pisolithes, amphore Dr. 23) couvre une surface d'environ 2 ha. Les témoins d'un habitat résidentiel sont signalés dans la partie centrale du site, par des fragments de tubuli, des briques d'hypocauste et par deux fragments de tegulae mammatae. On est tenté de reconnaître dans cette zone centrale un secteur résidentiel, faisant écho à un secteur agricole, dont la fouille a dégagé les vestiges: l'ensemble renvoie une image domaniale classique, celle d'une importante villa du Bas-Empire. Notons également, la présence de deux concentrations importantes de scories de fer, témoignant de l'importance de la métallurgie sur le site à cette période, largement prouvée du reste par les découvertes réalisées sur la fouille (infra, § 6).

\section{L'ÉTABLISSEMENT DU BAS-EMPIRE: L'AQUEDUC ET LES DERNIÈRES CONSTRUCTIONS DE LA VILLA} (phase C3, courant du $\mathrm{IV}^{\mathrm{e}} \mathrm{s}$. - début du $\mathrm{V}^{\mathrm{e}} \mathrm{s}$.)

L'occupation du Bas-Empire se poursuit avec dynamisme dans la seconde moitié du $\mathrm{IV}^{\mathrm{e}} \mathrm{s}$. et le début du $\mathrm{V}^{\mathrm{e}} \mathrm{s}$. La période n'enregistre pas de pauses, que ce soit dans l'activité de l'établissement ou dans les investissements consentis pour son développement. Bien au contraire, les réalisations sont nombreuses: un nouvel agrandissement des bâtiments agricoles, et surtout la construction d'un aqueduc souterrain.

\subsection{L'aqueduc (fig. 36 à 46)}

Comme nous l'avons signalé en introduction, l'aqueduc de Mayran a été découvert lors de travaux agricoles. Ces derniers mirent en évidence un regard, assez semblable dans sa morphologie à un puits, dont le curage révéla qu'il communiquait, à $4,5 \mathrm{~m}$ de profondeur, avec un aqueduc souterrain, s'écoulant du sud vers le nord (fig. 36). Ce dernier a pu faire l'objet d'observations plus poussées à l'occasion de la fouille.

Les décapages ont permis de repérer la tranchée d'implantation de la canalisation sur plus de $160 \mathrm{~m}$ (fig. 37). Cette tranchée n'est plus perceptible vers le nord (zone 2) car l'aqueduc apparaît ici dès la surface, du fait de la déclivité du terrain naturel. Dans ce même secteur, à l'aboutissement de la section maçonnée de l'aqueduc, un bassin réceptionne les eaux de ce dernier. Celui-ci a connu différentes étapes de construction que nous détaillerons plus loin. En aval de ce bassin, la conduite d'eau se présente de façon beaucoup plus modeste sous la forme d'une canalisation en céramique dont on ignore la destination finale.

Afin d'analyser les différentes parties de cet ouvrage, plusieurs sondages ont été ouverts. Dans la partie amont de l'aqueduc, au niveau des bâtiments de la villa (zone 1), deux sondages profonds ont été pratiqués jusqu'à la canalisation (1.1 et 1.2, fig. 37). Dans la partie aval (zone 2), les aménagements repérés autour du bassin ont été fouillés. Deux sondages ont également été pratiqués sous la chape du bassin (2.2 et 2.1), permettant d'étudier les états antérieurs de cette construction, de même qu'un sondage sur la partie terminale de la canalisation maçonnée (2.3). Afin de confirmer l'extension de la canalisation vers le nord, un sondage mécanique a été réalisé en limite de parcelle (2.4).

L'importance et l'originalité de l'aménagement hydraulique de Mayran justifiaient qu'on l'étudie dans le détail. Cet aqueduc présente en effet des caractéristiques inhabituelles, à commencer par la profondeur de son installation (en amont) et les grandes dimensions de son specus, qui le distinguent des autres aqueducs domaniaux connus dans la région. 


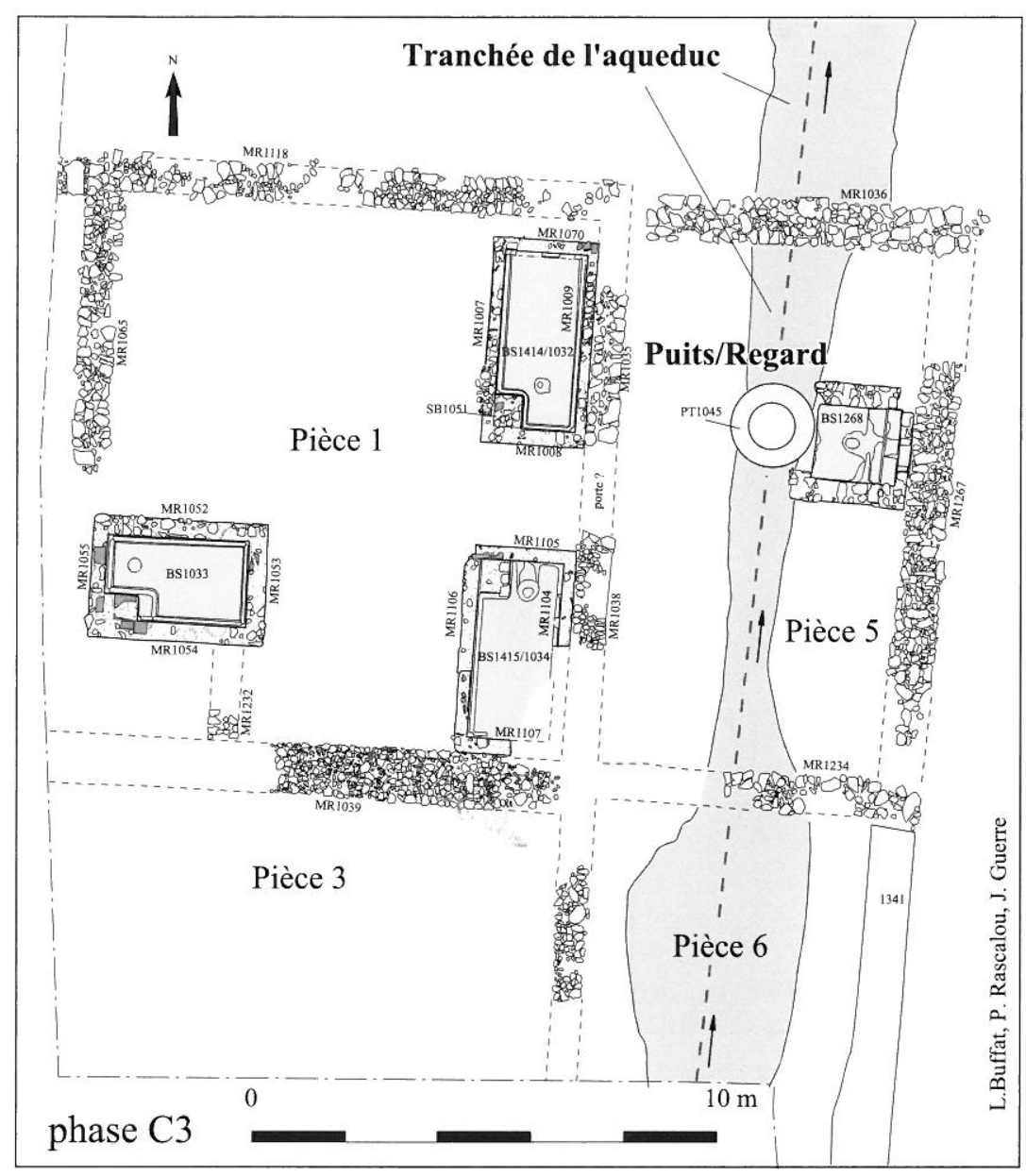

Fig. 36. Les nouvelles constructions de la phase C3.

C'est l'époque de construction de l'aqueduc, installé dans cette zone a près de $4 \mathrm{~m}$ de profondeur. Deux nouvelles pièces (5 et 6 ) sont construites après l'installation de l'aqueduc. Les bâtisseurs ont pris soin de réduire la largeur de la tranchée de l'aqueduc au niveau des cuves, probablement pour ne pas déstabiliser celles-ci. Le chai ne semble pas connaître de modifications, autant qu'on puisse en juger vu l'état de conservation des constructions.

la terre, acheminement des matériaux, accès à la tranchée. On ne retrouve pas ce type d'aménagement sur le bord occidental, plus régulier et plus droit.

La canalisation maçonnée: morphologie et techniques de construction

La canalisation présente des dimensions qui varient assez peu (fig. 37, 38). En amont (zone 1 ), la dimension du specus est à peu près constante: dans le sondage $1.1,0,85 \times 0,47 \mathrm{~m}$, dans le sondage $1.2,0,9 \times 0,47 \mathrm{~m}$, soit, dans les deux cas, environ 3 pieds sur 1,5. En aval, au contact du bassin septentrional (2.3), la largeur est identique, mais la profondeur supérieure $(1,1 \mathrm{~m})$. Cependant la conduite présente ici un surcreusement de $0,35 \mathrm{~m}$, assurément occasionné par l'érosion hydraulique (infra, 5.2.2.).

On observe des variations dans la mise en

\subsubsection{La partie amont de l'aqueduc: la canalisation maçonnée}

\section{Les travaux de construction}

Les observations effectuées lors de la fouille apportent quelques précisions sur le chantier de construction de la canalisation. La mise en place de celle-ci a nécessité le creusement d'une tranchée nord-sud, dont la profondeur atteint, au niveau de la villa, environ $5 \mathrm{~m}$. Cette tranchée a été reconnue sur une longueur de $162 \mathrm{~m}$. Dans la partie amont (zone 1), la tranchée longe la façade orientale des bâtiments agricoles, sans causer de destructions apparentes. En plan, elle présente une largeur qui varie entre 1 et $3 \mathrm{~m}$. Elle se rétrécit notablement au niveau des installations vinicoles, dans la pièce 5, vraisemblablement pour ne pas déstabiliser les constructions situées à l'ouest (fig. 36).

Sondée à deux reprises au niveau des bâtiments antiques (fig. 37 et 38,1.1 et 1.2), la tranchée présente un profil intéressant. Le bord oriental, évasé, comporte des ressauts, particulièrement nets dans le sondage 1.2. Dans ce cas, on distingue deux "marches" surplombant la galerie. Ces ressauts pourraient correspondre à des paliers, destinés à faciliter le travail des bâtisseurs : évacuation de œuvre du plancher de la canalisation. En amont, dans le sondage 1.1, le fond est constitué de grandes dalles. En revanche, dans le sondage 1.2 et, en aval, dans le sondage 2.3, le fond correspond au substrat pliocène. Dans ce cas, on peut penser que les bâtisseurs n'ont pas dallé le fond en raison de la présence d'encroûtements solides et compacts (sables agglomérés, formant des plaques), suffisant certainement à assurer l'étanchéité.

Les murs qui délimitent la conduite maçonnée sont constitués d'assises irrégulières de blocs liés au mortier. Les jambages sont plus soignés en amont (sondages 1.1 et 1.2 ), où des blocs plus réguliers ont été utilisés. Les différents tronçons fouillés n'ont livré aucune trace d'un enduit, opus signinum ou autre. En amont, où la canalisation est profondément enfouie, de grandes dalles (en grès), rectangulaires, d'environ 1 x 0,8 ont été utilisées pour la couverture. Des variations dans la qualité de cette couverture sont à noter. Les dalles du sondage 1.2 sont soigneusement jointoyées au mortier, tandis que celles du sondage 1.1 n'étaient colmatées que par des petites pierres calcaires et des fragments de tegulae (fig. 38). Ceci pourrait relever d'un souci "d'économie", qui n'est pas rare dans la construction des aqueducs romains (Andrieu 1995, 138). Ce pourrait également être un dispositif de drainage 


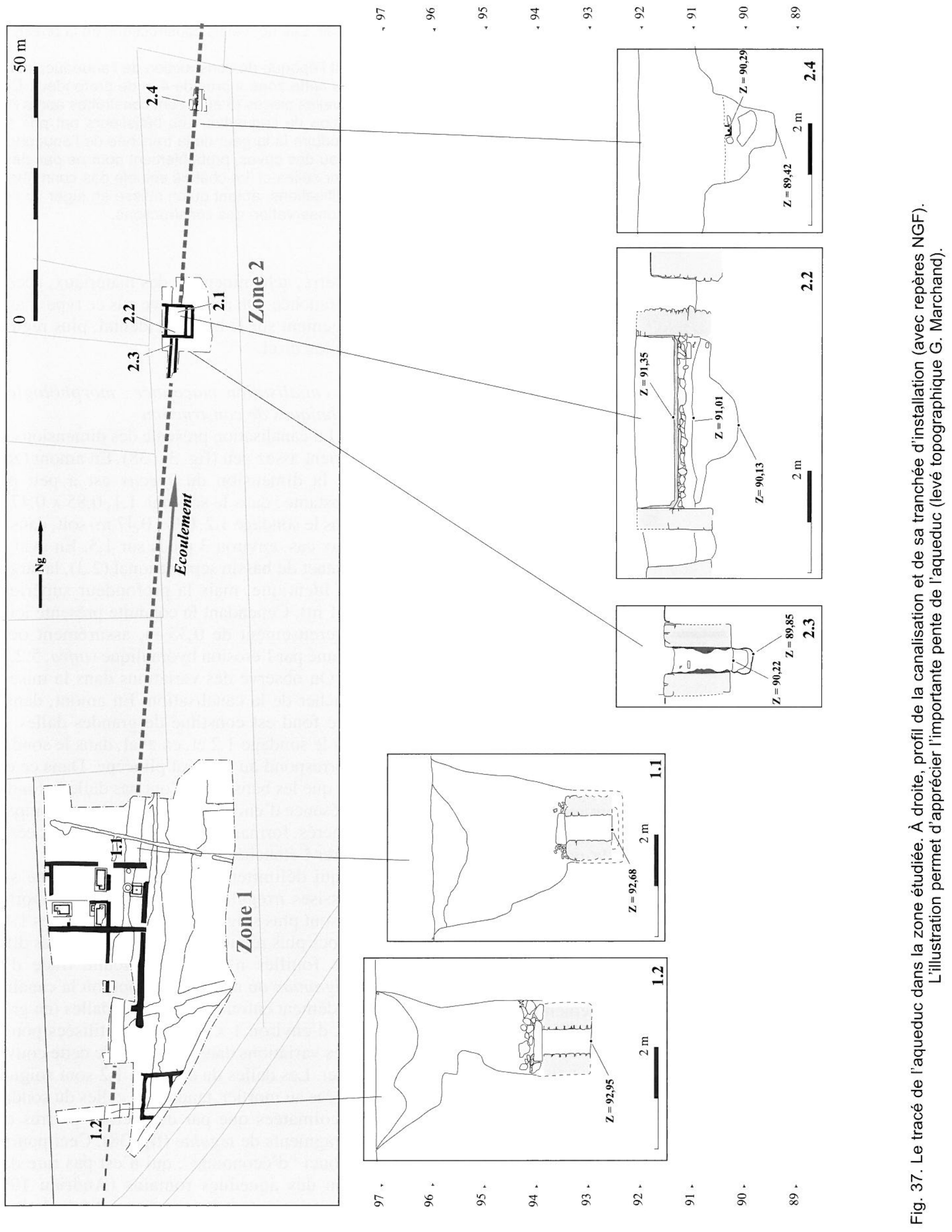




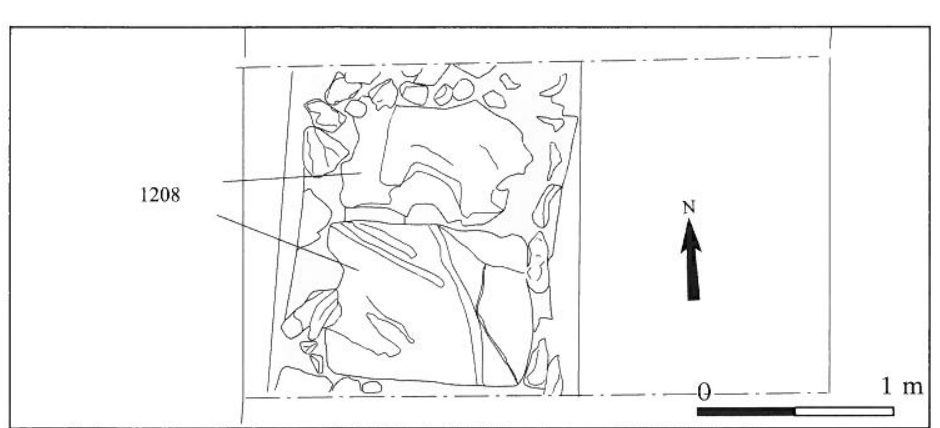

Couverture de l'aqueduc dans le sondage 1.2

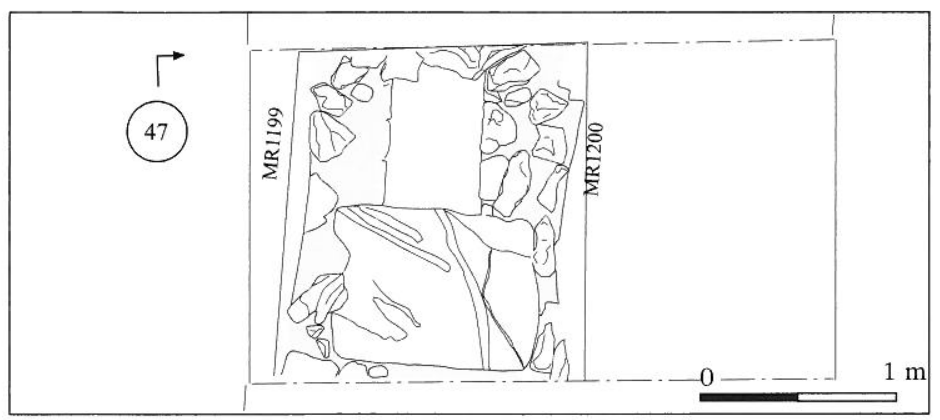

L'aqueduc après enlèvement d'une dalle de couverture (sondage 1.2)

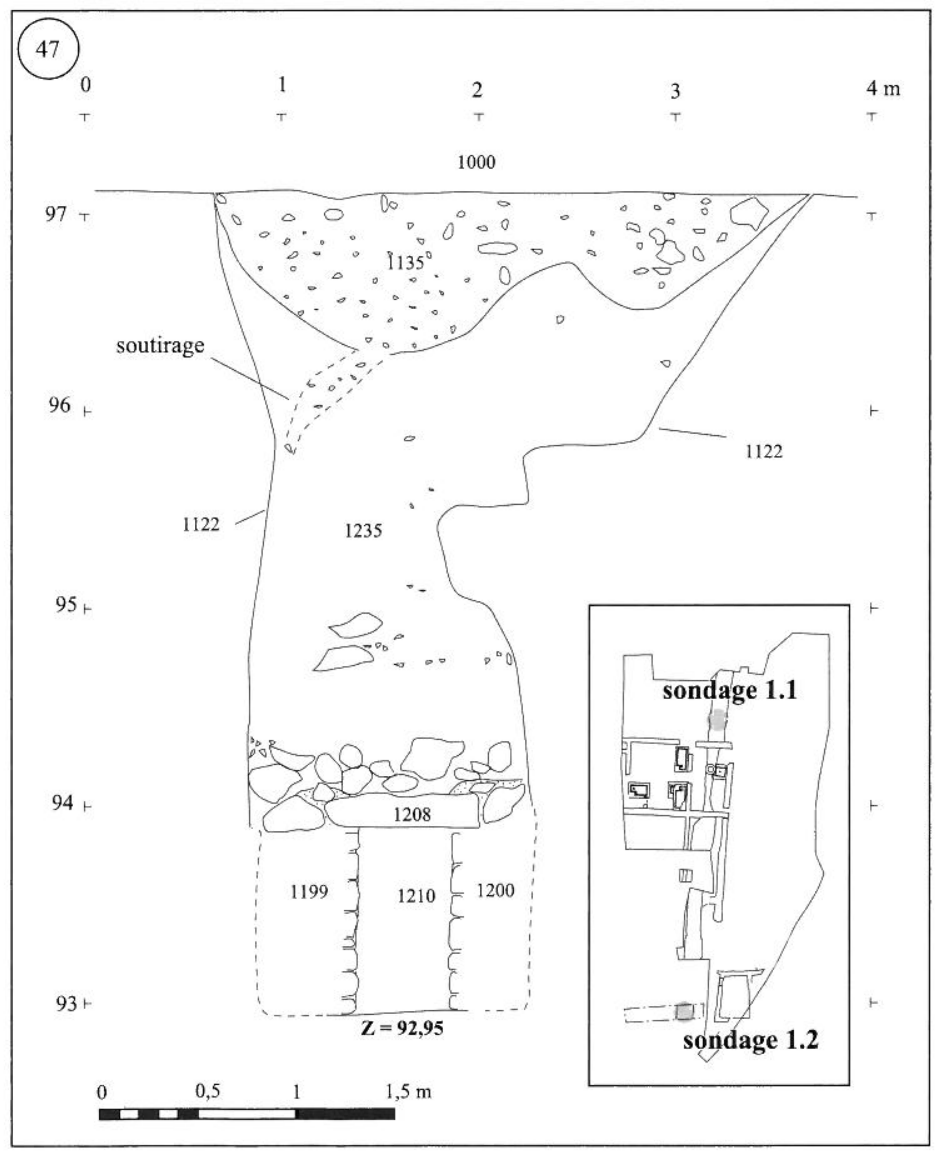

Coupe de l'aqueduc et de sa tranchée d'installation (sondage 1.2)

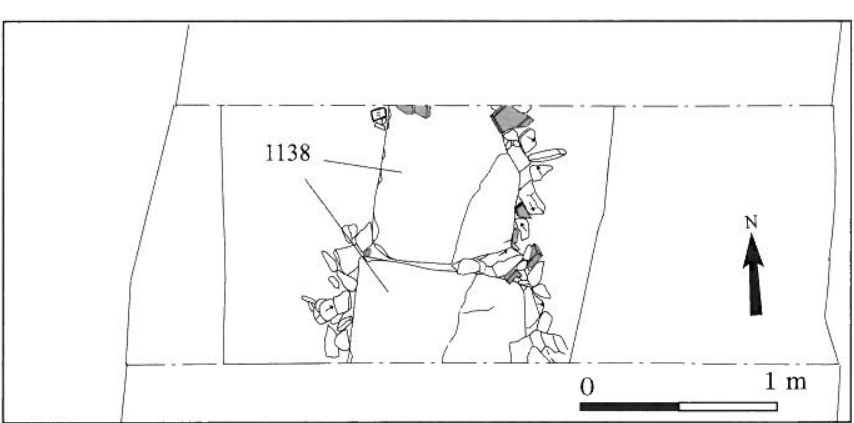

Couverture de l'aqueduc dans le sondage 1.1

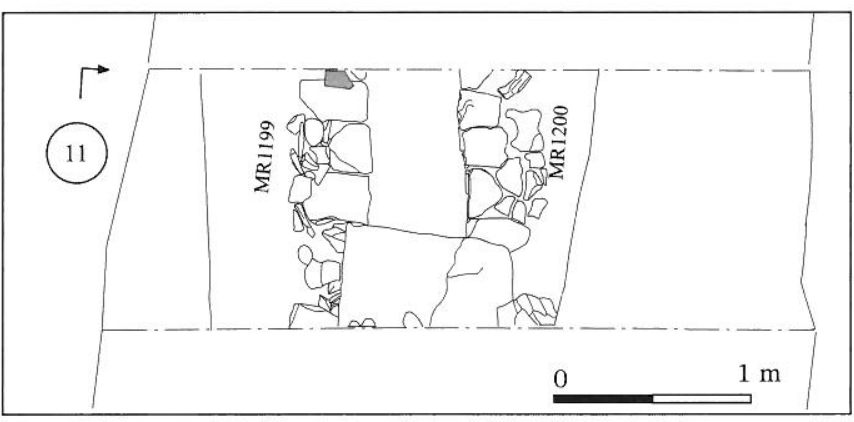

L'aqueduc après enlèvement d'une dalle de couverture (sondage 1.1)

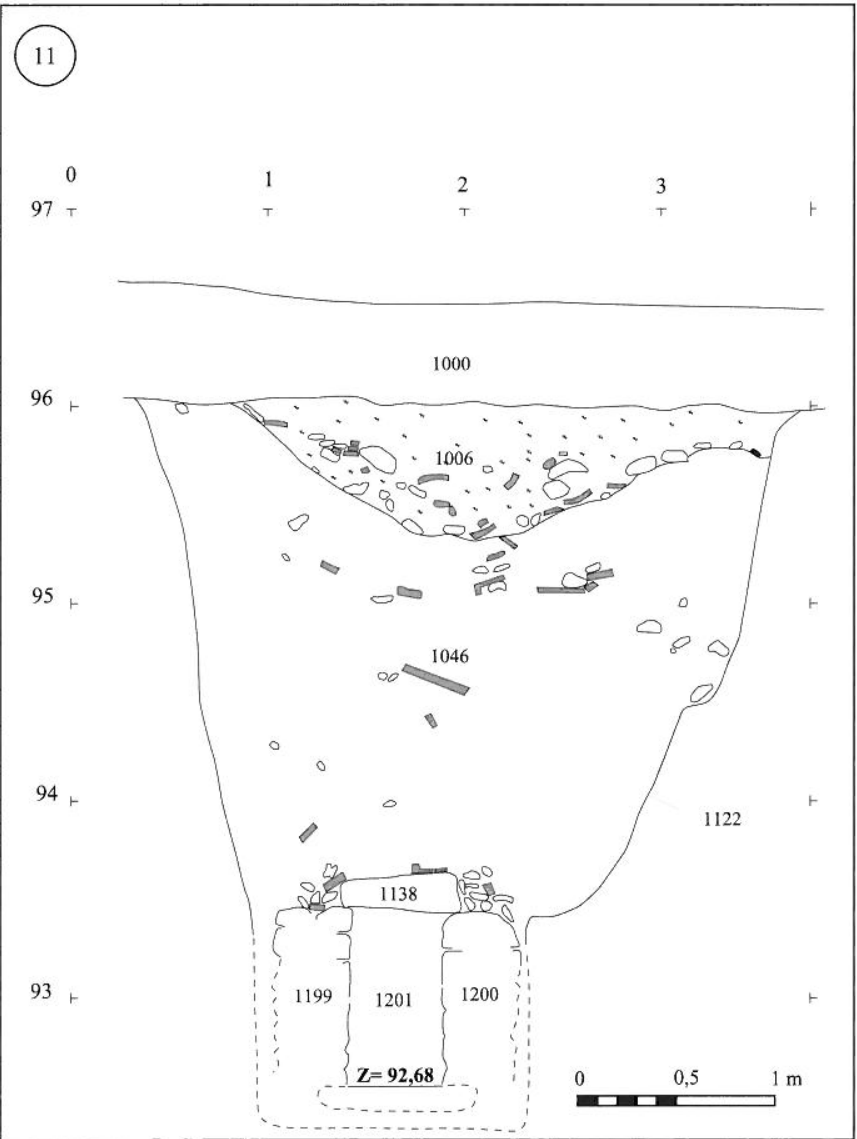

Coupe de l'aqueduc et de sa tranchée d'installation (sondage 1.1)

Fig. 38. Plan et coupes des deux sondages réalisés sur l'aqueduc.

Les coupes, recalées en NGF, permettent d'apprécier le pendage important de cette conduite d'eau. On perçoit bien, dans le sondage 2, un emmarchement destiné à faciliter l'évacuation de la terre (relevé : H. Petitot, J. Guerre; calage NGF : G. Marchand). 

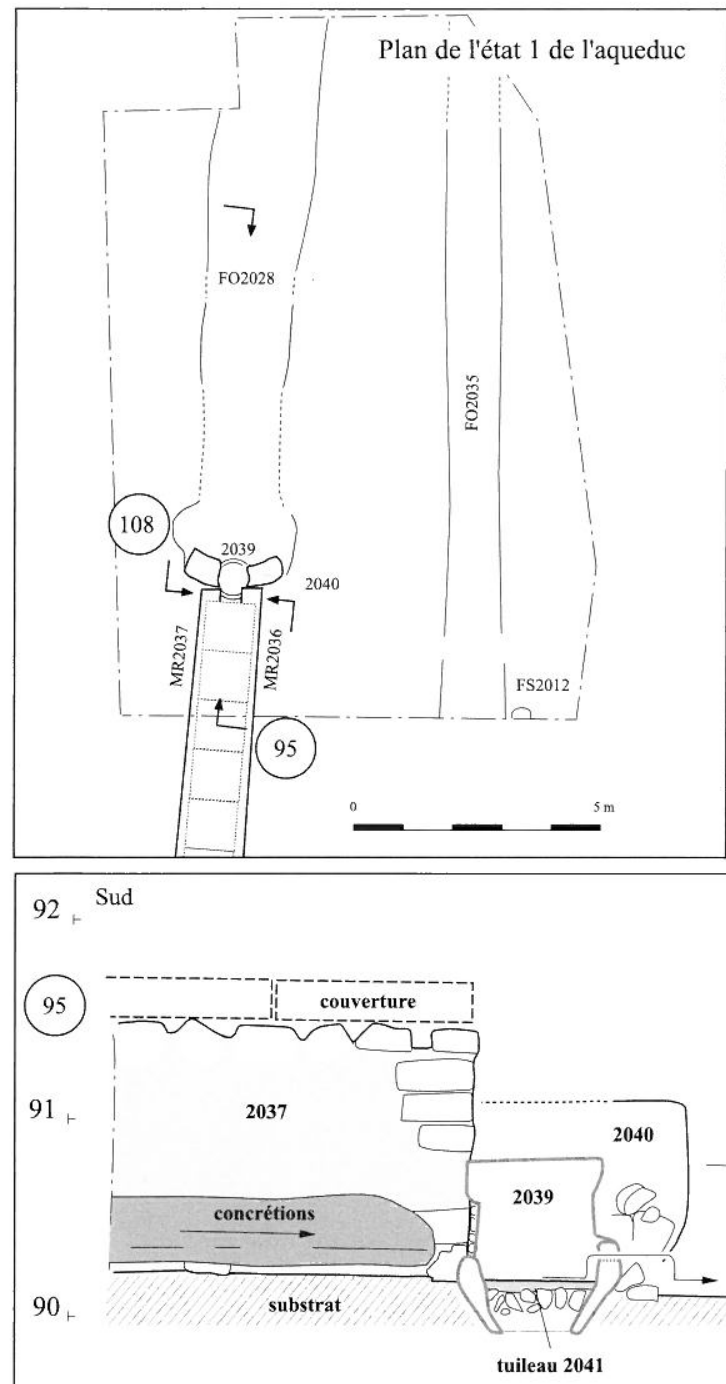

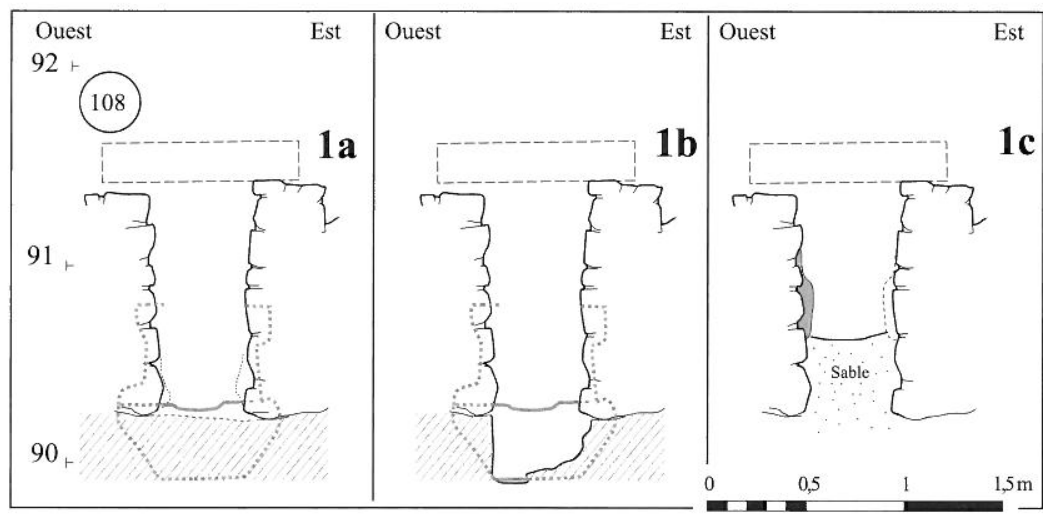

Evolution de l'exutoire de l'aqueduc au cours de l'état 1 (Relevé de terrain : B. Robin-Petitot)

Fig. 39 


\section{ci-contre}

Fig. 39 - Plan et coupe des aménagements repérés dans la zone 2 : le premier état.

La section souterraine de l'aqueduc aboutit à un aménagement maçonné, remployant une meta asinaria renversée, à l'intérieur de laquelle une chape de tuileau a été coulée. Au-delà de cette construction, l'eau s'écoule dans un simple fossé. L'activité de l'aqueduc apparaît stable dans un premier temps (1a). Dans un second temps, la zone qui précède la meta est surcreusée, probablement par le courant (1b). Dans un troisième temps, la canalisation s'ensable et son fil d'eau remonte d'une cinquantaine de $\mathrm{cm} \mathrm{(1c)}$.

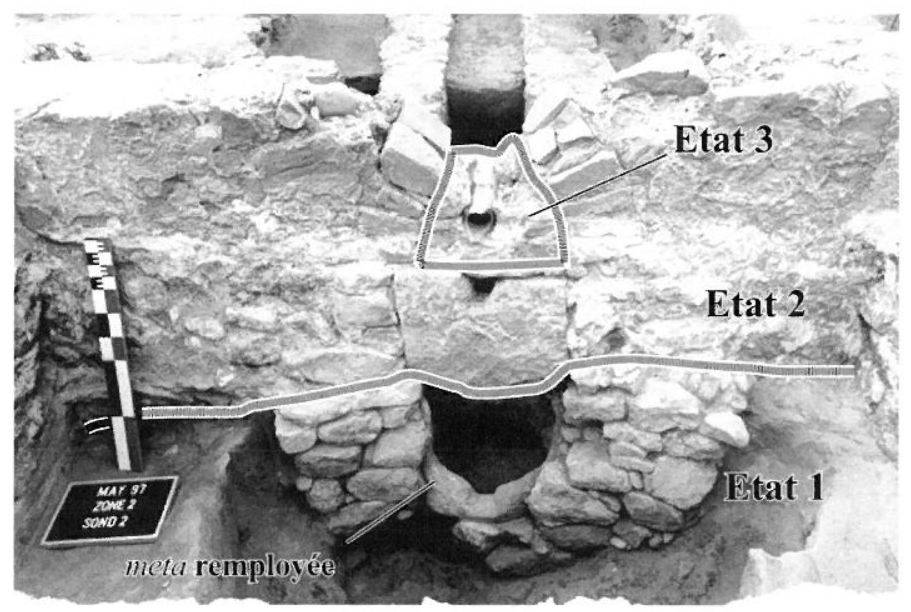

Fig. 40. Les aménagements successifs réalisés à la terminaison de la section souterraine de l'aqueduc. Vue du nord (cliché H. Petitot).

des eaux souterraines. Quoi qu'il en soit, il est clair que la canalisation a été victime d'infiltrations de sables. À plusieurs endroits, des dépressions sont présentes à la surface de la tranchée d'installation. Elles sont apparemment dues à des phénomènes de soutirage du comblement initial (fig. 38, sondage 1.2). Certaines de ces dépressions ont été comblées au $\mathrm{V}^{\mathrm{e}} \mathrm{s}$. par des déchets d'activité métallurgique (infra, § 6.1.). On ne peut émettre aucune observation en ce qui concerne la couverture du tronçon aval (2.3), les dalles ayant été récupérées dans les parties que nous avons étudiées.

\subsubsection{La partie aval de l'aqueduc: évolution et vicissi- tudes d'un aménagement hydraulique}

Au nord du chantier, la section maçonnée de l'aqueduc aboutit à un bassin dont la fouille a permis de détailler l'évolution.

\section{- État 1 (fig. 39)}

Durant la phase initiale, le bassin n'existe pas encore sous une forme maçonnée. L'aqueduc aboutit à un aménagement rudimentaire constitué d'une meta asinaria ren-

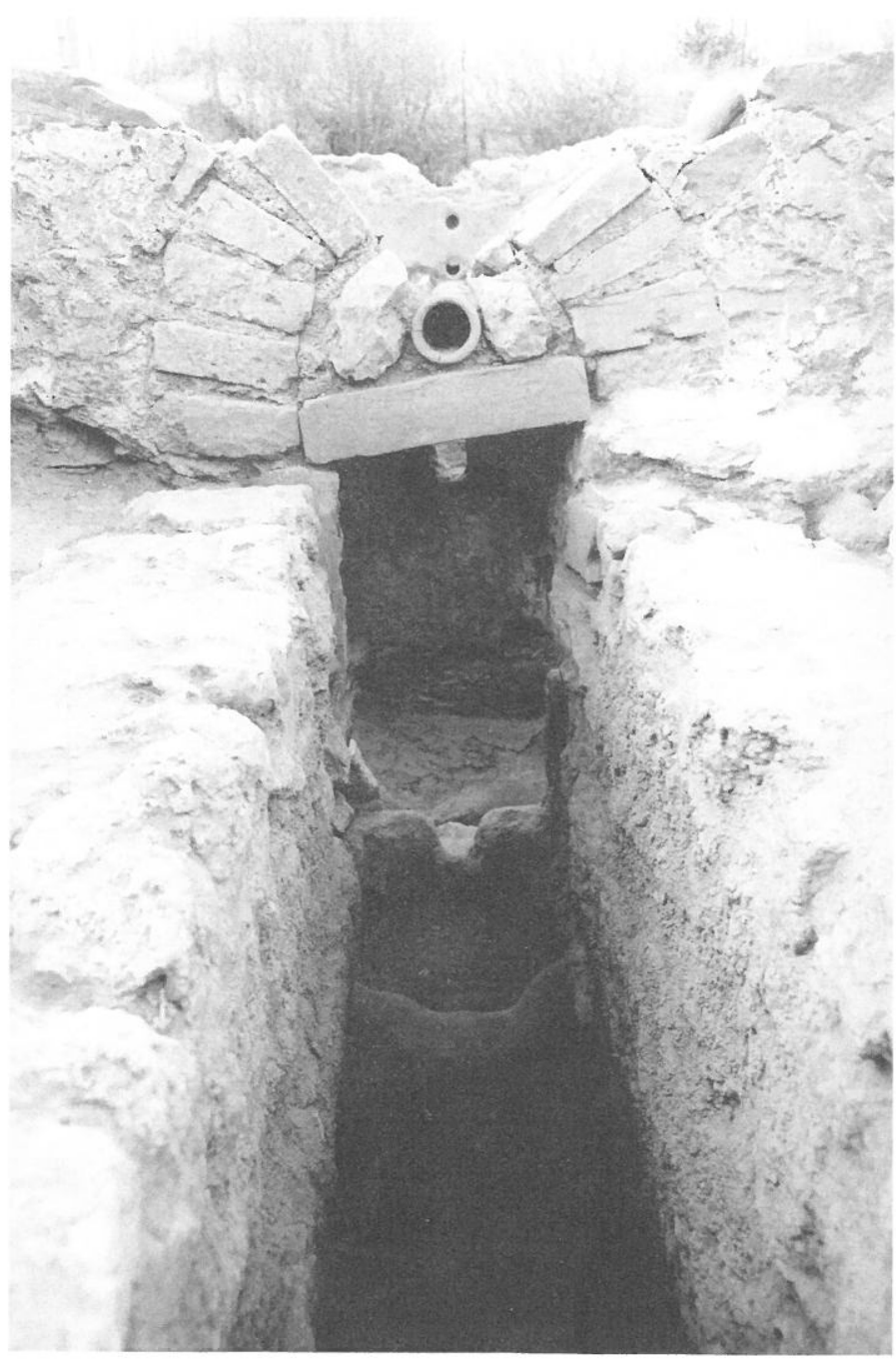

Fig. 41. Les aménagements présents à la terminaison de la section souterraine de l'aqueduc vus du sud. En bas, la meta remployée (état 1). En haut, les constructions du bassin avec l'embouchure coiffée d'un arc en plein cintre (état 2). Cet arc a été postérieurement bouché, l'arrivée d'eau étant désormais assurée par un simple tuyau en céramique.

versée (partie conique en bas) entourée par deux murets affectant une forme semi-circulaire (fig. 39, 40). Au centre de la meta, une chape de béton de tuileau a été coulée, constituant une petite cuvette qui comprend au nord un petit déversoir. Autour de cet élément de remploi, deux murets sont construits assez grossièrement. Au-delà, on trouve un grand fossé profond d'environ $0,6 \mathrm{~m}$. Ce fossé affecte, dans les premiers mètres, une forme irrégulière résultat de l'érosion hydraulique plus forte (turbulences). Il prend ensuite une forme plus régulière. Les sondages réalisés en aval révèlent qu'il se poursuivait sur une longue distance, au-delà de la parcelle investie.

Les données de la fouille montrent que le fil d'eau de l'aqueduc a changé au moins trois fois durant cet état (fig. 39). Détaillons rapidement cette évolution. Le plus 


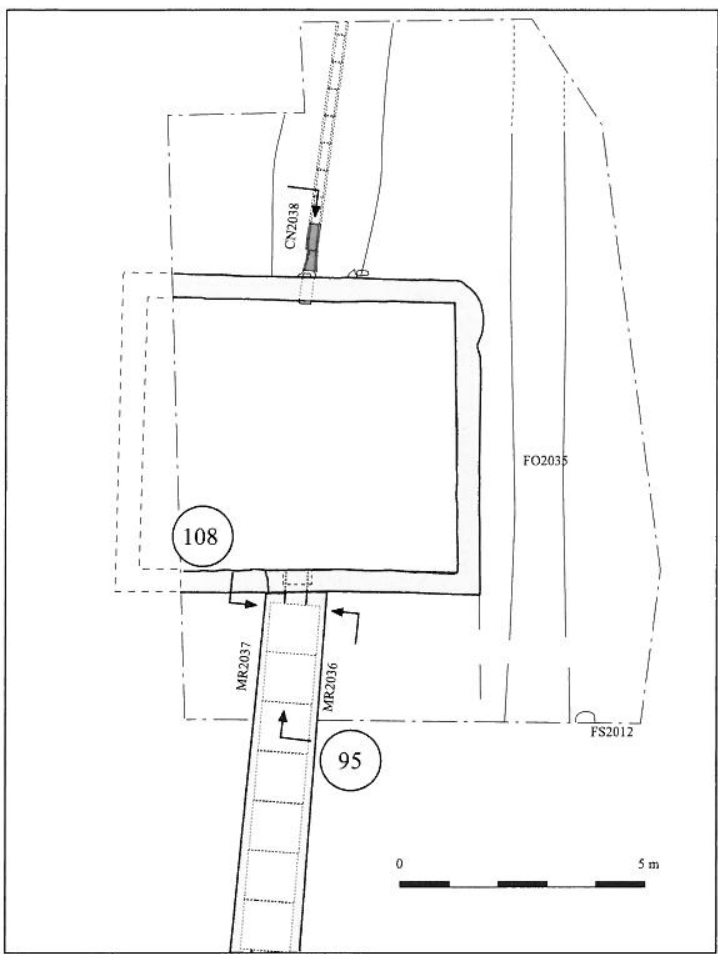

L'aqueduc et le bassin durant l'état 2

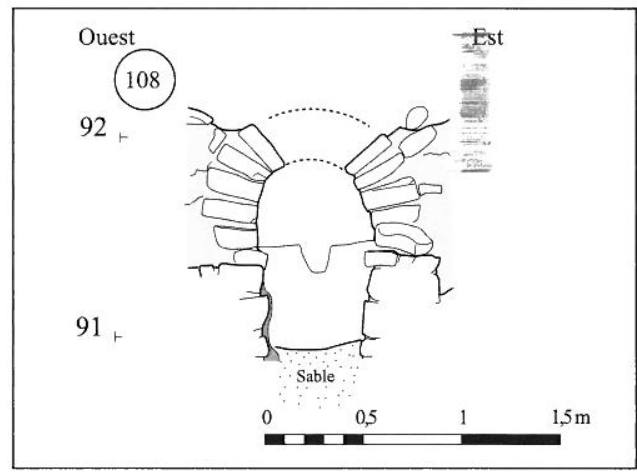

L'embouchure du bassin durant l'état 2

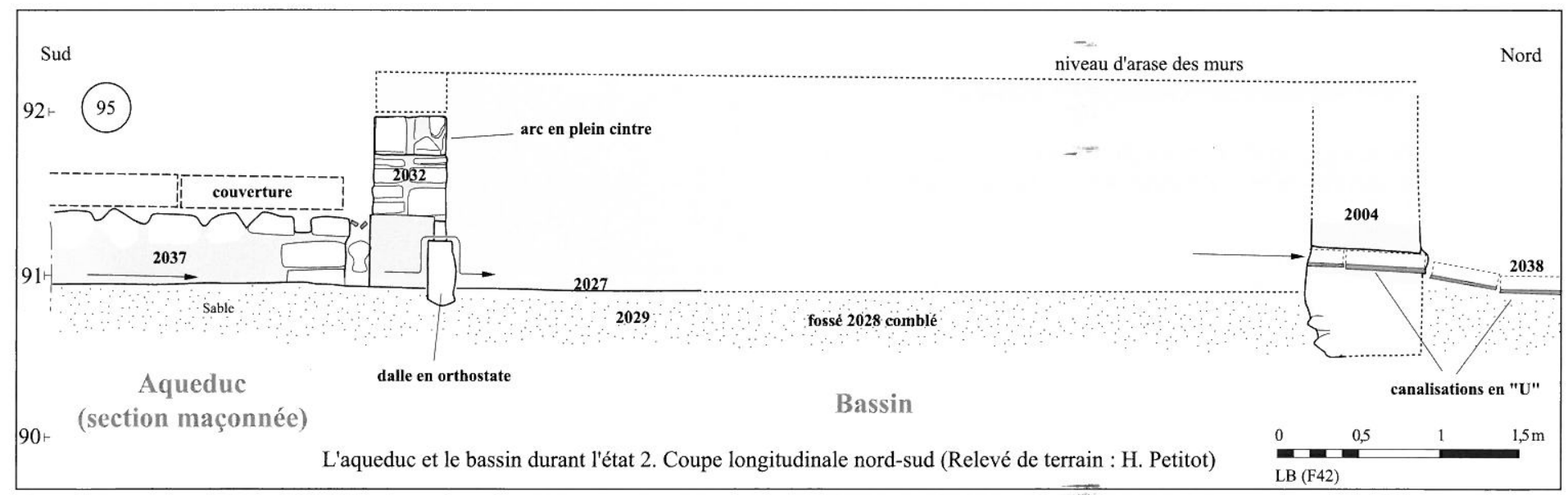

Fig. 42. Plan et coupe des aménagements repérés dans la zone 2 deuxième état.

L'ensablement a une nouvelle fois provoqué une accrétion du fil d'eau. Un bassin est construit en remplacement des aménagements antérieurs. Fait assez surprenant il ne comprend pas de sol en dur. Son embouchure correspond à un arc en plein cintre constitué de claveaux en calcaire coquillier. Sous cet arc, un bloc été disposé, de chant. II comprend dans sa partie haute une petite encoche qui faisait office de déversoir. Le bassin se déverse dans une petite canalisation constituée de canalisations en "U". Celles-ci sont installées bout à bout, sans liant, sur le comblement de l'ancien fossé, en grande partie ensablé. Ce dispositif se prolonge au moins jusqu'à la limite nord du chantier.

ancien fil d'eau se situait au même niveau que la base des murs de l'aqueduc (état 1a). Le débit moyen semble modeste si l'on en juge par la faible hauteur des dépôts carbonatés qui marquent ce premier fonctionnement. La vasque formée par la meta servait alors de petit bac de décantation. La seconde étape de fonctionnement (1b) est marquée une forte activité érosive. Celle-ci se traduit, en amont de la meta, par un surcreusement progressif du fond de de la canalisation, sur une trentaine de $\mathrm{cm}$. À certains endroits (quand le substrat est friable) la canalisation affecte un profil en "trou de serrure", caractéristique de cette érosion hydraulique (Bazzana et al. 1987, 53, 54, fig. 5b). La troisième phase (1c) est marquée par un ensablement important de la canalisation qui entraîne une 
remontée du fil d'eau de près de $0,7 \mathrm{~m}$. Le concrétionnement qui accompagne cette étape de fonctionnement présente une hauteur importante $(0,35)$ qui suggère des écoulements forts et réguliers.

\section{- État 2 (fig. 42)}

Au premier dispositif, modeste, se substitue un bassin de plan rectangulaire mesurant 6,6 x 5,5 m (dans œuvre). Conservés sur 1,1 m de hauteur, les murs de ce bassin sont constitués par des assises irrégulières de blocs liés au mortier (fig. 40, état 2). Dans son état conservé, ce bassin présente une capacité d'une quarantaine de $\mathrm{m}^{3}$. Fait assez surprenant il ne comprend pas de sol en dur. Son embouchure est soigneusement aménagée. Elle correspond à un arc en plein cintre constitué de claveaux en calcaire coquillier (fig. 41). Sous cet arc, un bloc été disposé, de chant. Il comprend dans sa partie haute une petite encoche qui faisait office de déversoir.

À cette période, le fil d'eau de l'aqueduc est sensiblement remonté (fig. 42). L'accumulation du sable a comblé le specus sur près de $0,8 \mathrm{~m}$. La section d'écoulement s'est trouvée réduite à $0,45 \times 0,5 \mathrm{~m}$. À l'embouchure du bassin, l'eau se heurtait à la dalle en orthostate, qui servait vraisemblablement de premier seuil de décantation (fig. 40). À l'intérieur du bassin, il est assez surprenant de ne pas retrouver trace d'un sol en dur, mais le sol limoneux pourrait être suffisamment imperméable. On observe comme seul aménagement une fine chape de mortier (2027), mais celle-ci pourrait correspondre à un niveau du chantier de construction du bassin.

Au nord, l'eau s'écoulait du bassin pour emprunter une canalisation en terre cuite à profil en $U$, prise dans la maçonnerie (fig. 42). Elle se déverse dans une petite conduite, constituée par la juxtaposition d'éléments identiques. Ces derniers sont installés bout à bout, sans liant, sur le comblement de l'ancien fossé, en grande partie colmaté. Les sondages menés en aval du bassin (2.4, fig. 37) attestent l'existence de cette canalisation en céramique jusqu'à la limite septentrionale du chantier (fig. 43). Les éléments qui la constituent sont en pâte calcaire rosée à dégraissant rare. Ils sont tous de même dimension, 0,53 x $0,21 \mathrm{~m}$, avec une section intérieure d'écoulement de 0,16 x $0,1 \mathrm{~m}$. On notera qu'ils ne bénéficient pas de dispositif d'emboîtement. Des canalisations semblables étaient produites à l'atelier de Sallèles d'Aude (Laubenheimer 1990, 104, 107, n¹6). Elles différent cependant par la présence d'un système d'emboîtement. Elles sont également un peu plus longues : 0,61 m en moyenne. Mais on notera que si l'on retranche la longueur de l'emboîtement, soit $0,07 \mathrm{~m}$, on retrouve une valeur équivalente à celle des canalisations de Mayran. Ce système de canalisation en "U" est assez fréquent dans les aqueducs domaniaux de la région. Un tronçon de l'aqueduc du Puech Quinau, situé non loin de Béziers (Hérault), comporte des éléments de ce type. Il est constitué d'éléments céramiques, longs de $0,86 \mathrm{~m}$,

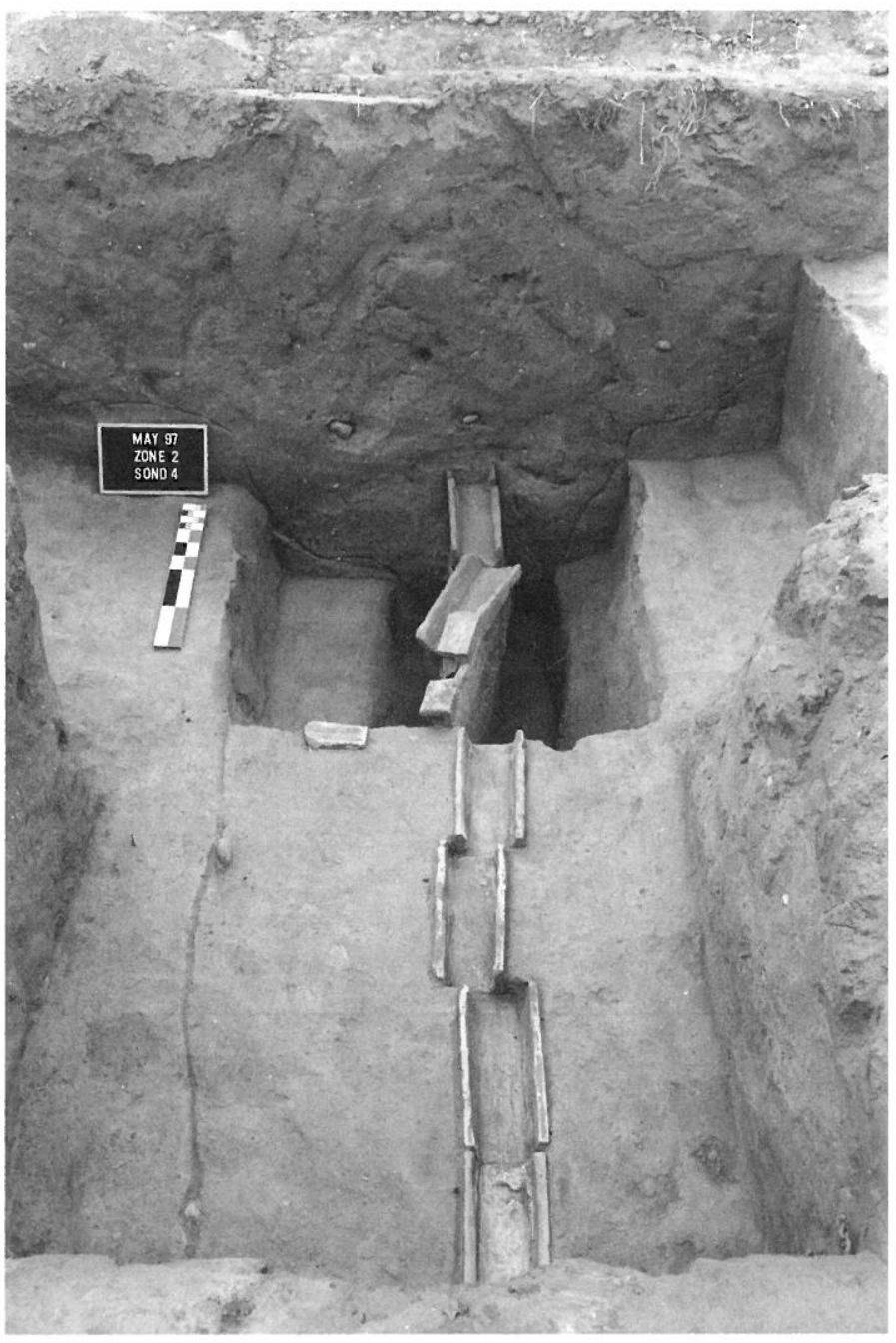

Fig. 43. Canalisation en terre cuite aménagée sur le comblement du fossé antérieur (cliché $\mathrm{H}$. Petitot).

posés sur un lit de mortier et recouverts d'une dalle épaisse de $5 \mathrm{~cm}$. La section intérieure d'écoulement $(0,11$ x $0,11 \mathrm{~m}$ ) pouvait transiter au mieux 2 litres/seconde d'après J.-L. Andrieu (1999, 75, 99, cliché 2). Près de Narbonne à Bizanet (Aude), M. et R. Sabrié ont étudié un aqueduc domanial, comprenant notamment un pont aqueduc, qui utilise aussi des canalisations en U pour son parcours enterré (Pellecuer 2000, 28). À Mayran, aucun élément ne venait couvrir le canal en céramique, contrairement à Puech Quinau ou Bizanet. Apparemment l'eau pouvait librement déborder de la canalisation.

La section d'écoulement de ce tronçon d'aqueduc en céramique $(0,16 \times 0,1 \mathrm{~m})$ paraît très réduite. Mais à considérer d'autres aqueducs domaniaux, elle n'a finalement rien d'inhabituel. L'aqueduc maçonné d'el Khous en Algérie dispose d'un canal de 0,28 $\mathrm{m}$ de large pour $0,18 \mathrm{~m}$ de haut (Leveau 1984, 253-254, 422-423). L'aqueduc de Loupian, est large d'environ 0,2 $\mathrm{m}$, pour une hauteur de l'ordre de 0,2 à 0,4 m (Pellecuer 2000, 26, 422, fig. 11-18). 


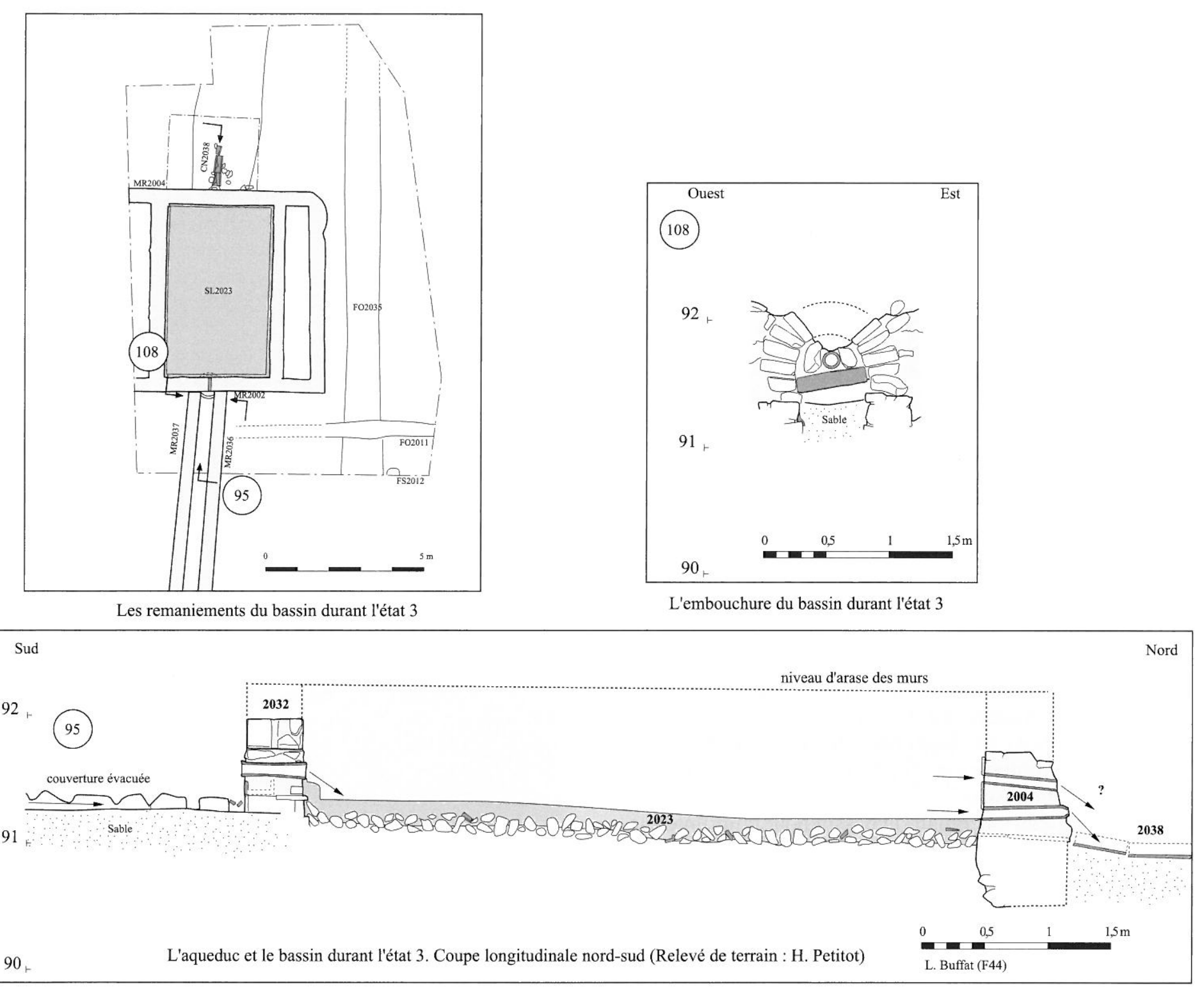

Fig. 44. Plan et coupe des aménagements repérés dans la zone 2 : le dernier état (3).

Une nouvelle accrétion du fil d'eau oblige à démonter les dalles de couverture. L'eau circule désormais dans un simple fossé. Le bassin est réduit par la construction de nouveaux murs. Le fond du bassin reçoit un sol de tuileau, les parois sont enduites de béton hydraulique. L'arrivée d'eau ainsi que les évacuations sont constituées de tuyaux en céramique de section circulaire.

\section{- État 3 (fig. 44)}

La mise en place de nouveaux murs et d'un sol en béton de tuileau dans le bassin constitue les principales modifications du troisième état (fig. 44). Ces nouveaux murs ramènent la surface du bassin à des dimensions plus modestes $(3,35 \times 5,4 \mathrm{~m})$. Les parois et les fonds sont enduits par du béton hydraulique en opus signinum. L'étanchéité, au niveau du contact fond-paroi, est assurée par un boudin en quart de rond. L'enduit de paroi, relativement bien conservé, présente une épaisseur de $2-3 \mathrm{~cm}$. La chape de fond, épaisse de $8 \mathrm{~cm}$, est coulée sur un hérissonnage de pierres et de galets. Elle présente une pente assez marquée, du sud vers le nord $(15 \mathrm{~cm}$ de dénivelé entre les deux extrémités du bassin).

Lors de ces travaux, l'embouchure en plein cintre du bassin est oblitérée par de la maçonnerie (fig. 44). Au centre de ce bouchage, un tuyau circulaire a été disposé et sert de nouvel orifice d'admission d'eau. Sa hauteur suggère que le fil d'eau est remonté très haut, plus haut d'ailleurs que le sommet des murs bajoyers de l'aqueduc. À cette période, les dalles de couverture ont certainement été enlevées et l'eau ne circulait plus ici dans l'aqueduc, mais dans un simple fossé. L'eau devait alors transporter une quantité importante de sable, ce qui explique peut-être 

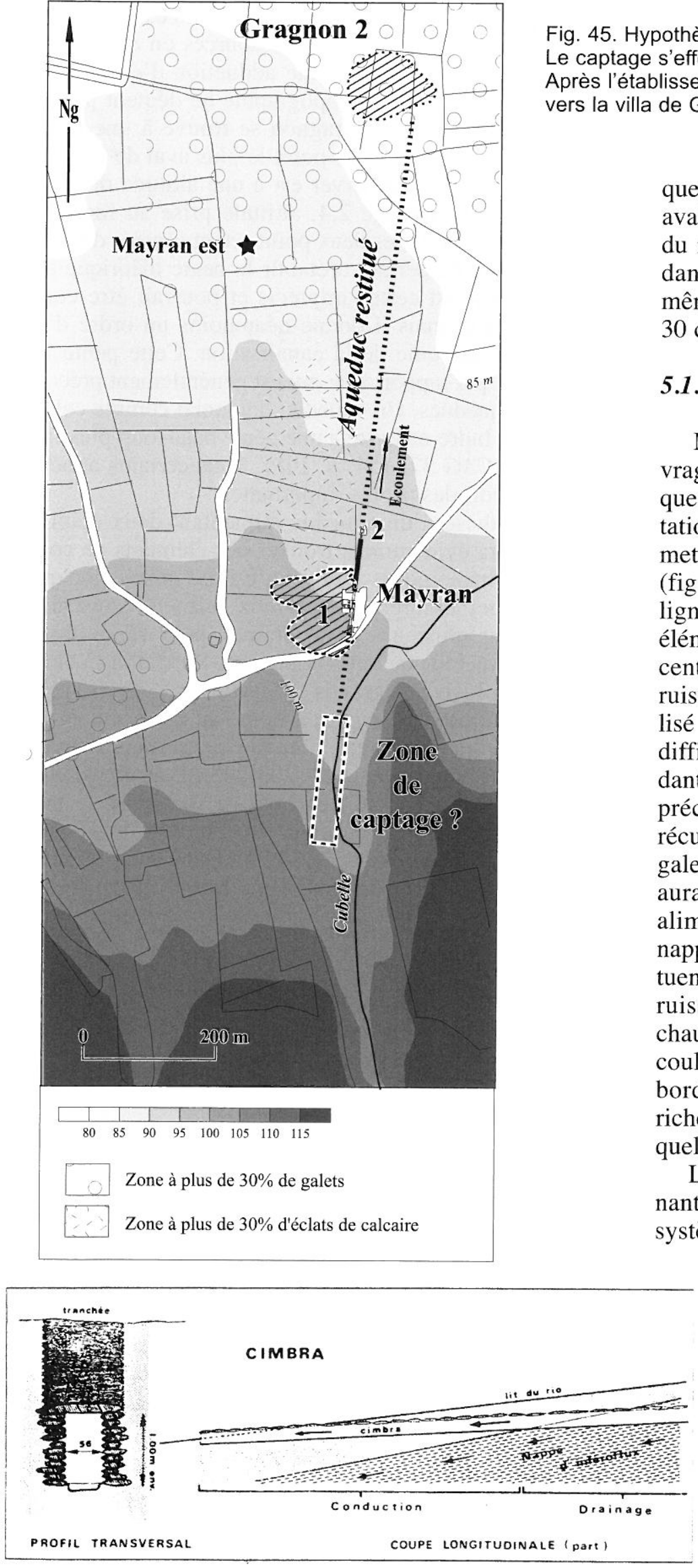

Fig. 45. Hypothèse de restitution du tracé de l'aqueduc de Mayran. Le captage s'effectuerait dans le lit du ruisseau de Cubelle. Après l'établissement de Mayran, la canalisation se dirige vers la villa de Gragnon 2.

que l'embouchure ait été réduite à ce modeste tuyau. En aval, l'évacuation a également été modifiée. Deux tuyaux, du même type que celui de l'embouchure, sont installés dans la partie centrale du mur. Le premier se situe au même niveau que le fond du bassin. Le second est installé $30 \mathrm{~cm}$ plus haut.

\subsubsection{L'alimentation en eau (fig. 45,46 )}

Maintenant que l'on a présenté les vestiges de l'ouvrage hydraulique de Mayran, il convient d'aborder des questions plus générales, en particulier celle de l'alimentation en eau. Le calage précis du tracé de l'aqueduc permet de proposer une localisation pour la zone de captage (fig. 45). En admettant que la galerie suive un tracé rectiligne, en amont du tronçon étudié (hypothèse qu'aucun élément ne contredit), l'alimentation peut être resituée une centaine de mètres plus haut, là où la galerie rejoint le ruisseau de Cubèle (fig. 45). Mais quel système était utilisé pour récupérer l'eau? En l'absence de fouille, il est difficile d'avoir quelques certitudes. On retiendra cependant que la profondeur de l'ouvrage répond à un besoin précis. Une telle profondeur pourrait se justifier par la récupération des eaux souterraines, grâce à un système de galerie drainante. Pourquoi une telle solution technique aurait-elle été retenue? Probablement pour assurer une alimentation en eau continue en captant directement la nappe. À l'heure actuelle, les eaux superficielles constituent une ressource instable: en saison froide, le débit du ruisseau de Cubèle est faible et irrégulier, en saison chaude, il est nul. Par contre, si les eaux superficielles ne coulent qu'occasionnellement, le ruisseau est aujourd'hui bordé par une végétation vivace qui témoigne de la richesse hydraulique souterraine. Un puits actuel, creusé à quelques mètres du ruisseau, atteint l'eau à $2 \mathrm{~m}$.

Le captage pourrait donc se faire par une galerie drainante, suivant en souterrain le lit du ruisseau. C'est un système qui était utilisé dans l'Espagne médiévale pour l'alimentation des acequias (fig. 46). Les galeries drainantes qui alimentaient ces acequias, les cimbras, étaient installées dans le lit des cours d'eau. De faible pendage, elles récupéraient la nappe d'inféroflux (Bazzana et al. 1987, 54-56, fig. 7). Dans leur morphologie, les tronçons de galerie observés à Mayran présen-

Fig. 46. Principe de captage des cimbras (Bazzana et al. 1987, 56, fig. 7). 
tent de grandes ressemblances avec ces cimbras: " une longue tranchée, excavée dans le lit du rio, voit sa base renforcée de deux murets de pierre sèche de faible hauteur (env. $1 \mathrm{~m}$ ) puis recouverts de dalles de schiste. La partie supérieure de l'ouvrage est ensuite comblée. La tête de la galerie ainsi ménagée est la partie active du système, celle où l'eau de la nappe s'écoule par gravité, pour être enfin acheminée par le canal de base. Elle se trouve alors à une profondeur de l'ordre de 4 à $5 \mathrm{~m}$ sous la surface du rio » (Ibid., 54). La seule différence que l'on peut observer ici correspond à la mise en œuvre des jambages du canal, qui à Mayran sont liés au mortier, non en pierre sèche. Cette technique de galerie drainante était déjà connue à l'époque romaine (Ibid., 57; Fabre et al. 1999, 198-199).

\subsubsection{Utilisation et entretien}

L'aqueduc de Mayran devait réclamer un entretien constant. Les infiltrations de sable et autres limons, importantes d'après les données de la fouille, impliquent de fréquents curages. Le puits installé sur la canalisation n'est pas étranger à cette pratique, puisqu'il permet, entre autres, d'accéder à la galerie pour procéder à des nettoyages. Il pouvait évidemment exister d'autres ouvertures sur le tracé de l'aqueduc, mais la fouille n'en a pas révélé. Le puits servait aussi à récupérer de l'eau pour les besoins de la villa. Aucun élément n'autorise à restituer le système de puisage. L'absence de la margelle originelle et la disparition des niveaux d'occupation interdisent de trancher entre les solutions possibles: pompe, appareillage à poutres et chèvre, puisage au seau...

La présence d'un cuveau à l'est de la margelle du puits (fig. 36, 1268) atteste un stockage temporaire de l'eau. La présence d'une cupule montre qu'il faisait office de bac de décantation, mais aucun aménagement annexe ne vient nous renseigner sur les détails de son fonctionnement.

\subsubsection{Un aqueduc pour deux établissements?}

De nombreuses questions restent ouvertes en ce qui concerne cet aqueduc, à commencer par les raisons qui ont poussé les constructeurs à installer la canalisation aussi profondément. Au niveau du captage, l'aqueduc se situerait à $7 \mathrm{~m}$ de profondeur. On a proposé qu'il s'agisse d'un système destiné à capter directement l'eau dans la nappe. Mais on peut également y voir un choix technique, retenu pour diminuer au maximum la pente du canal. Un point important doit être considéré dans ce cadre. La conduite prend, au-delà des limites de la villa, la direction d'un autre établissement gallo-romain situé $800 \mathrm{~m} \mathrm{au}$ nord, l'établissement de Gragnon 2 (fig. 45). Il s'agit d'une villa assez étendue (1,5 ha), occupée du I ${ }^{\text {er }}$ jusqu'au $\mathrm{V}^{\mathrm{e}} \mathrm{s}$. En limite est de ce gisement, une dalle en pierre calcaire d'1,2 x 0,5 m a été observée. Par ses dimensions, elle est proche des dalles de couverture de l'aqueduc. On signalera également que le site en question est implanté sur des terrasses de galets où les ressources en eau sont très faibles. L'aménagement d'une adduction d'eau ne serait donc pas surprenant. La topographie ne dément pas cette hypothèse: la villa de Gragnon se trouve à une altitude d'environ $82 \mathrm{~m}$ NGF et le point le plus aval de l'aqueduc que nous avons pu observer est à une altitude de 90,3 m NGF (fig. 37, sondage 2.4, altitude prise au fond de la canalisation en U). Les deux points étant séparés de $600 \mathrm{~m}$, un calcul rapide permet d'établir la pente théorique à 1,3 $\%$. Ce calcul est certes imprécis et pourrait être corrigé dans le détail, mais il donne néanmoins un ordre d'idée valable sur la pente de la canalisation. Cette pente reste importante par rapport à ce qui est généralement préconisé pour les aqueducs. Un aqueduc domanial comme celui de Luynes en Indre-et-Loire a une pente beaucoup plus faible $(0,15 \%$ - CAG 37, 154, p. 108). Mais certains aqueducs peuvent avoir des pentes importantes.

L'hypothèse d'un aqueduc alimentant deux établissements devra évidemment trouver des éléments de confirmation archéologiques. Mais il n'est pas impossible qu'un tel ouvrage, assurément coûteux, ait été construit et financé par plusieurs propriétaires, pour répondre aux besoins respectifs de leurs domaines. Rappelons à ce propos que plusieurs grands propriétaires de la région nîmoise ont probablement participé au financement d'un ouvrage hydraulique autrement plus important, l'aqueduc de Nîmes (Fabre et al. 1999, 203-205). Pour la vallée des Baux, P. Bellamy et R. Hitchner se sont posé le même type de question concernant les moulins de Barbegal (Bellamy, Hitchner 2000, 228-229). Dans une perspective un peu différente, reconnaissons aussi qu'il n'est pas exclu que l'une de ces deux villae soit à un moment donné passée sous contrôle de l'autre, selon un schéma de concentration foncière pressenti par certains archéologues (cf. par exemple Brun in $C A G 83 / 1$, p. 154). Un même propriétaire aurait pu dès lors bâtir une canalisation pour deux établissements de son fundus.

\subsubsection{Datation de l'ouvrage}

Le mobilier qui date la construction de l'aqueduc reste peu abondant. Quelques indices attestent clairement qu'il s'agit d'une construction tardive: un fr. de bol en céramique claire engobée (forme B11), présent dans le comblement de la tranchée (us 1046, sondage 1.1), un bord de plat en céramique à pisolithes (forme $\mathrm{C} 2 \mathrm{c}$ ) retrouvé dans la tranchée de fondation du jambage est de l'aqueduc (sondage 2.3). Ce dernier élément conduit à fixer le terminus post quem de l'aqueduc au milieu du $\mathrm{IV}^{\mathrm{e}} \mathrm{s}$., la céramique à pisolithes n'apparaissant sur les sites de consommation que dans la seconde moitié du $\mathrm{IV}^{\mathrm{e}} \mathrm{s}$. (Raynaud in Py 1993, 527).

Les autres éléments sont insuffisants pour entrer dans le détail chronologique des modifications de l'ouvrage. 
Des remblais constitués majoritairement de déchets métallurgiques sont venus dans le courant du $\mathrm{V}^{\mathrm{e}} \mathrm{s}$. combler des dépressions probablement occasionnées par un soutirage du comblement de la galerie (infra, § 6.1.), mais il reste difficile de mettre ces éléments en relation avec les mutations observées dans la partie nord de l'aqueduc, au niveau du bassin (zone 2).

Le mobilier recueilli dans le comblement du dernier état du bassin (état 3) date son abandon d'un large $V^{\mathrm{e}} \mathrm{s}$., probablement avant le dernier tiers de ce siècle, si l'on en croit l'absence de céramique kaolinitique réductrice.

\section{INVENTAIRE DU MOBILIER}

\section{Zone 1}

\section{Comblement de la tranchée d'installation}

1046 (350/400) phase C3 - sigillée sud-gauloise: 1 fr., claire engobée oxydante: $1 \mathrm{fr}$. (CL-ENG B11). Total céramiques: 2 fr.

Recharge du comblement

$1006(350 / 400)$ phase C3 - amphore hispanique: $1 \mathrm{fr}$. Total amphores: $1 \mathrm{fr}$.

1135 (350/400) phase C3 - sigillée sud-gauloise: 1 fr., kaol. réductrice: 1 fr., amphore hispanique: 1 fr., amphore africaine : $1 \mathrm{fr}$. Total céramiques: $4 \mathrm{fr}$.

\section{Zone 2}

État 1

\section{Construction}

2034 (calage du piédroit est de l'aqueduc) - piso. oxydante: $1 \mathrm{fr}$. (PISO C2c)

\section{Abandon}

2029 (comblement du fossé 2028) - amphore gauloise: 2 fr.

\section{État 2}

Pas de matériel

État 3

Construction

2025 (niveau de chantier du bassin) - commune indéterminée: $1 \mathrm{fr}$.

2023 (hérissonnage du tuileau du bassin) - amphore de Bétique :

$3 \mathrm{fr}$., amphore orientale : $1 \mathrm{fr}$. Total céramiques : $4 \mathrm{fr}$.

\section{Comblement aqueduc}

$2024(\mathrm{C} 3 \mathrm{c})$ - amphore gauloise: 2 fr., amphore africaine : $1 \mathrm{fr}$. Total céramiques : $3 \mathrm{fr}$.

Petit bouchon (pour canalisation?)

Abandon du bassin (comblement)

2016 (C3d) - claire récente: 1 fr., amphore gauloise: 1 fr., amphore gauloise sabl.: 1 fr. (G1), amphore hispanique: 3 fr. (A51c), amphore africaine: 11 fr., amphore indéterminée: $1 \mathrm{fr}$., total amphores: $17 \mathrm{fr}$. Total céramiques: $18 \mathrm{fr}$.

12 fr. de tuyau en céramique; os animaux : 1 ; éclats de silex : 2; bouchon en tuile: 1 ; demi-bouchon en tuile: 1

2018 (C3d) - claire récente: 23 fr. (plat indét.), piso. oxydante: 1 fr., amphore africaine: 3 fr., amphore indéterminée: 1 fr. Total céramiques : $28 \mathrm{fr}$.

2019 (C3d) - claire récente : 2 fr., amphore africaine : 1 fr. Total céramiques : 3

2020 (C3d) - amphore gauloise: $7 \mathrm{fr}$.

$2026(\mathrm{C} 3 \mathrm{c})$ - amphore indéterminée: $1 \mathrm{fr}$.

2033 (C3 indét.) - claire B : 1fr. (Portout 37a), kaol. oxydante:
2 fr., amphore africaine : 1 fr. Total amphores: 1 fr. Total céramiques : 4 fr.

2031 (C3) - amphore africaine : $2 \mathrm{fr}$.

2032 (C3) - claire B : 1 fr., claire engobée oxydante : 2 fr., oxydante micacée: 2 fr., kaol. réductrice: 1 fr., piso. oxydante: 1 fr., non tournée: 1 fr., amphore gauloise: 1 fr., amphore indéterminée: $1 \mathrm{fr}$. Total céramiques : $10 \mathrm{fr}$.

Plusieurs fr. de meule à leucites (meta asinaria)

2033 (C3) - claire B : 1 fr., kaol. oxydante : 2 fr., amphore africaine : $1 \mathrm{fr}$. Total céramiques: $4 \mathrm{fr}$.

Tige en fer: 2

\subsubsection{Conclusions}

Les travaux menés sur l'aqueduc de Mayran apportent des éclaircissements sur la morphologie et le fonctionnement de cet ouvrage. Son installation très en profondeur a de bonnes chances d'être liée à un captage des eaux souterraines du ruisseau de Cubèle. Les constructeurs ont probablement cherché à s'assurer un approvisionnement pérenne en captant directement la nappe. Cette hypothèse reste cependant à vérifier en ouvrant une fouille au niveau du ruisseau. Ceci permettra de valider l'hypothèse et d'analyser les solutions techniques retenues pour organiser ce drainage souterrain.

Les sondages pratiqués sur la canalisation, montrent que différentes solutions techniques ont été sollicitées pour la conduite de l'eau: dans les parties souterraines, on trouve un specus maçonné, solidement bâti. En revanche, sitôt que l'aqueduc retrouve un tracé aérien, on utilise des solutions plus simples et moins onéreuses: simple fossé dans un premier temps, puis petites canalisations en terre cuite. Mayran montre une nouvelle fois que les aqueducs domaniaux peuvent se réduire dans certains tronçons à des aménagements très simples, notamment dans des secteurs où les contraintes topographiques sont faibles. L'exemple de Mayran vient ainsi étoffer le corpus de ce que l'on pourrait appeler les aqueducs "multiformes" où alternent constructions importantes et dispositifs plus modestes (notamment des conduites en céramique). Il se rapproche en cela de quelques exemples régionaux, cités au fil du texte.

Les étapes de fonctionnement du bassin situé dans la partie aval de l'ouvrage mettent également en lumière les vicissitudes qu'a connues la canalisation tout au long de son fonctionnement. L'ensablement a représenté un problème constant. Le sable et les limons s'infiltraient dans la galerie, notamment au niveau des bâtiments de la villa où le comblement de la tranchée s'est progressivement tassé, par effet de soutirage. La responsabilité en revient peutêtre aux insuffisances du jointoiement de la couverture. Ceci n'a pas empêché l'eau de continuer à circuler, mais la section mouillée s'est progressivement réduite. À la fin de son utilisation, alors que le specus était totalement ensablé, la galerie continuait de drainer une partie de l'eau, par suintement. Aujourd'hui encore, la canalisation, 
Fig. 47

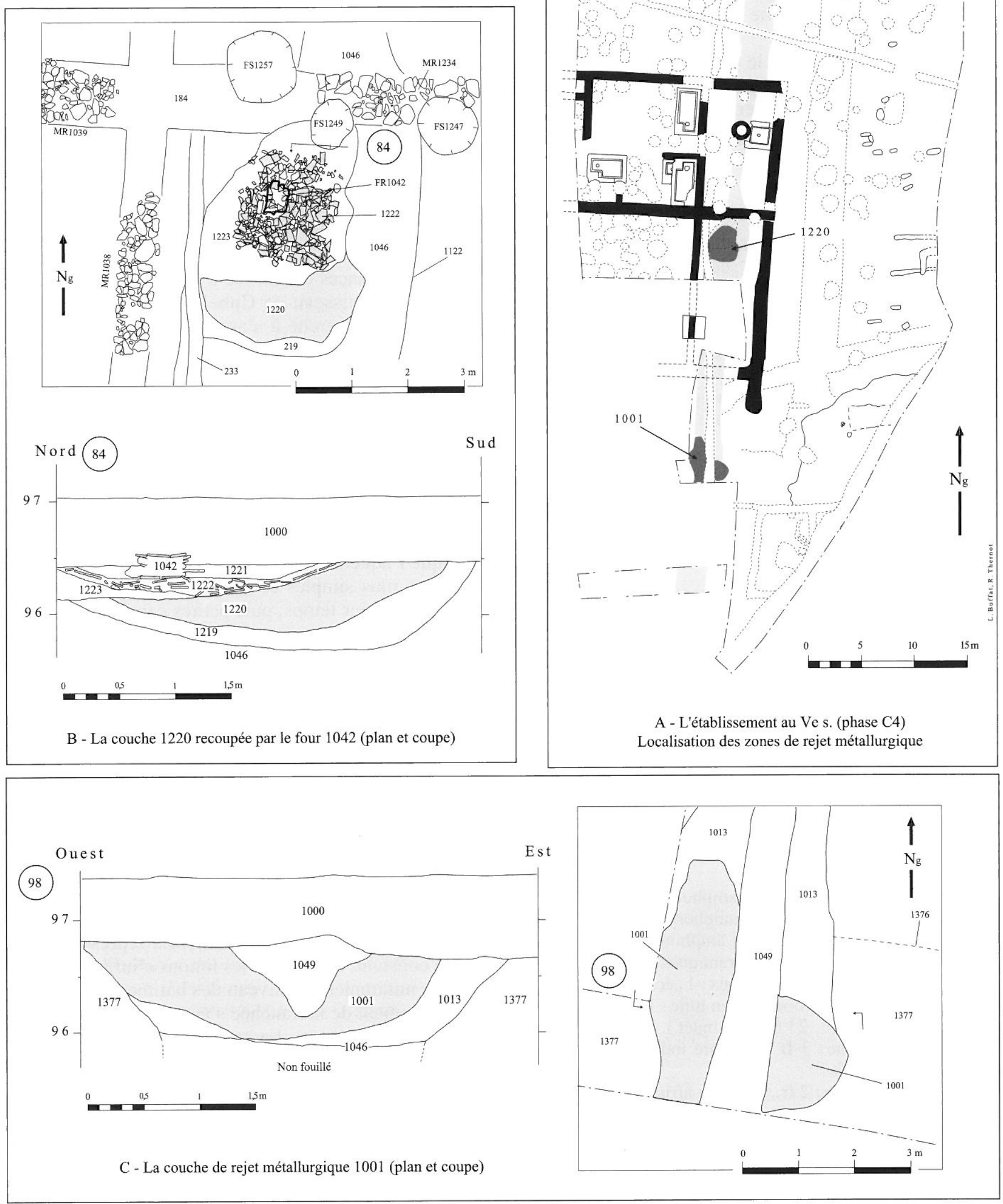




\section{ci-contre}

Fig. 47. Localisation, plans détaillés et coupes des fosses à déchets métallurgiques de la phase $\mathrm{C} 4\left(\mathrm{~V}^{\mathrm{e}} \mathrm{s}\right.$.).

bien que comblée, est partiellement en eau, notamment en période hivernale.

Les occupants du site ont cherché à lutter contre l'ensablement en aménageant un bassin de décantation. Celuici ne réglait pas le problème de l'ensablement de la partie amont du canal, mais il débarrassait l'eau d'une partie de ses impuretés, avant de la renvoyer vers l'aval. Incidemment, ce système de décantation montre que l'on prenait soin d'épurer cette eau quittant le périmètre des bâtiments antiques. Ce constat pourrait paraître bien inutile si la canalisation ne prenait ensuite la direction d'une autre villa située $800 \mathrm{~m}$ plus au nord. Celle-ci pourrait être alimentée par cet aqueduc.

On soulignera que la datation tardive de l'ouvrage (probablement la seconde moitié du $\mathrm{IV}^{\mathrm{e}} \mathrm{s}$.) montre, si besoin était, le dynamisme de l'établissement au BasEmpire. Fixer la durée de fonctionnement de l'ouvrage demeure par contre une entreprise délicate. Plusieurs indices tendent à montrer qu'il n'a pas fonctionné très longtemps. Une durée d'un siècle est une estimation raisonnable au vu des céramiques recueillies dans les différents niveaux. Ceci témoigne, malgré l'ampleur de l'investissement, des difficultés posées par la conception originelle de l'ouvrage, peut-être par son principe de captage. Trop exposé à l'ensablement, il n'a vraisemblablement pas rempli, sur le long terme, les espérances de ses concepteurs.

\subsection{De nouvelles constructions dans le bâtiment agricole (fig. 36)}

En ce qui concerne le bâtiment agricole, la principale évolution correspond à la mise en place de deux nouvelles pièces à l'est. Fort mal conservées, ces dernières n'ont pas livré d'éléments permettant de les interpréter (fig. 36). Seule la présence d'un puits donnant sur l'aqueduc et d'un cuveau confère à la pièce 5 une fonction particulière, en relation avec le puisage de l'eau dans l'aqueduc et la distribution de l'eau dans le bâtiment antique. Le mur de façade qui limite les deux nouvelles pièces à l'est est légèrement désaxé par rapport aux constructions antérieures, mais parfaitement parallèle au tracé de l'aqueduc. Les pièces affectent ainsi une forme légèrement trapézoïdale. Elles couvrent respectivement $60,5 \mathrm{~m}^{2}$ (pièce 5) et $77 \mathrm{~m}^{2}$ (pièce 6). Les élévations de murs étaient maçonnées comme en témoignent de rares traces de mortier sur le sommet des fondations.

\section{L'ÉTABLISSEMENT DU BAS EMPIRE: LES DERNIÈRES TRACES D'OCCUPATION ANTIQUE (phase $\mathrm{C} 4-\mathrm{V}^{\mathrm{e}}$ s. ap. J.-C.)}

Tant en fouille qu'en prospection de surface, l'établissement de Mayran n'enregistre pas de fléchissement de son occupation au $\mathrm{V}^{\mathrm{e}} \mathrm{s}$. Il n'y a plus cependant trace de nouvelles constructions. Mais les structures de la partie agricole mises en place précédemment, continuent de fonctionner. L'aqueduc connaît à cette époque des problèmes d'ensablement, que nous avons analysés précédemment. Même si la canalisation se colmate et que son débit se trouve réduit, elle fournit toujours de l'eau. Dans l'emprise des bâtiments agricoles, on voit se développer de nouvelles activités, notamment le travail du métal, qui occupe apparemment une place importante.

\subsection{Une importante activité métallurgique (fig. 47, 48)}

C'est dans l'enceinte de la partie agricole que deux couches particulièrement riches en scories de fer ont été exhumées. Attribuables à la fin du Bas-Empire, ces niveaux de rejet sont probablement à mettre en relation avec une forge, située hors de l'emprise de la fouille ou dont les vestiges ont été détruits par les travaux agricoles.

\section{Description des unités stratigraphiques}

- La couche 1001

La couche 1001 se situe dans la partie sud du chantier (fig. 47). Elle comble sur une longueur d'environ $4 \mathrm{~m} \mathrm{la}$ partie supérieure de la tranchée de l'aqueduc souterrain (1137). Elle prend place en un point où le remplissage initial $(1013,1046)$ du creusement semble s'être ponctuellement affaissé. Elle est recoupée dans sa partie centrale par un drain manifestement moderne (1049). La couche 1001 est composée d'une terre charbonneuse noire incluant une forte quantité de scories $(63 \mathrm{~kg}$ recueillis dans la partie fouillée). Ces scories se rattachent au type le plus fréquemment rencontré sur les chantiers de fouille: gangues ferreuses hétérogènes en forme de calotte comportant une multitude d'alvéoles. Les plus grosses peuvent atteindre une longueur de $20 \mathrm{~cm}$ et peser jusqu'à $1 \mathrm{~kg}$. Certaines conservent encore l'empreinte circulaire de la tuyère du four. 3 d'entre elles ont piégé des fragments de céramique à pâte calcaire, qui pourraient, malgré leur finesse $(0,5 \mathrm{~cm})$, constituer les restes du conduit de soufflerie. Outre ces scories, la couche livre quelques petites coulées de bronze (total: 7) présentant des sections franches.

\section{- Le comblement 1220}

La couche 1220 se trouve dans la partie centrale de la zone 1 du chantier. Comme la couche 1001, elle constitue une recharge dans le comblement de la tranchée de l'aqueduc (1137) dont le remplissage initial (1013/1046) paraît 


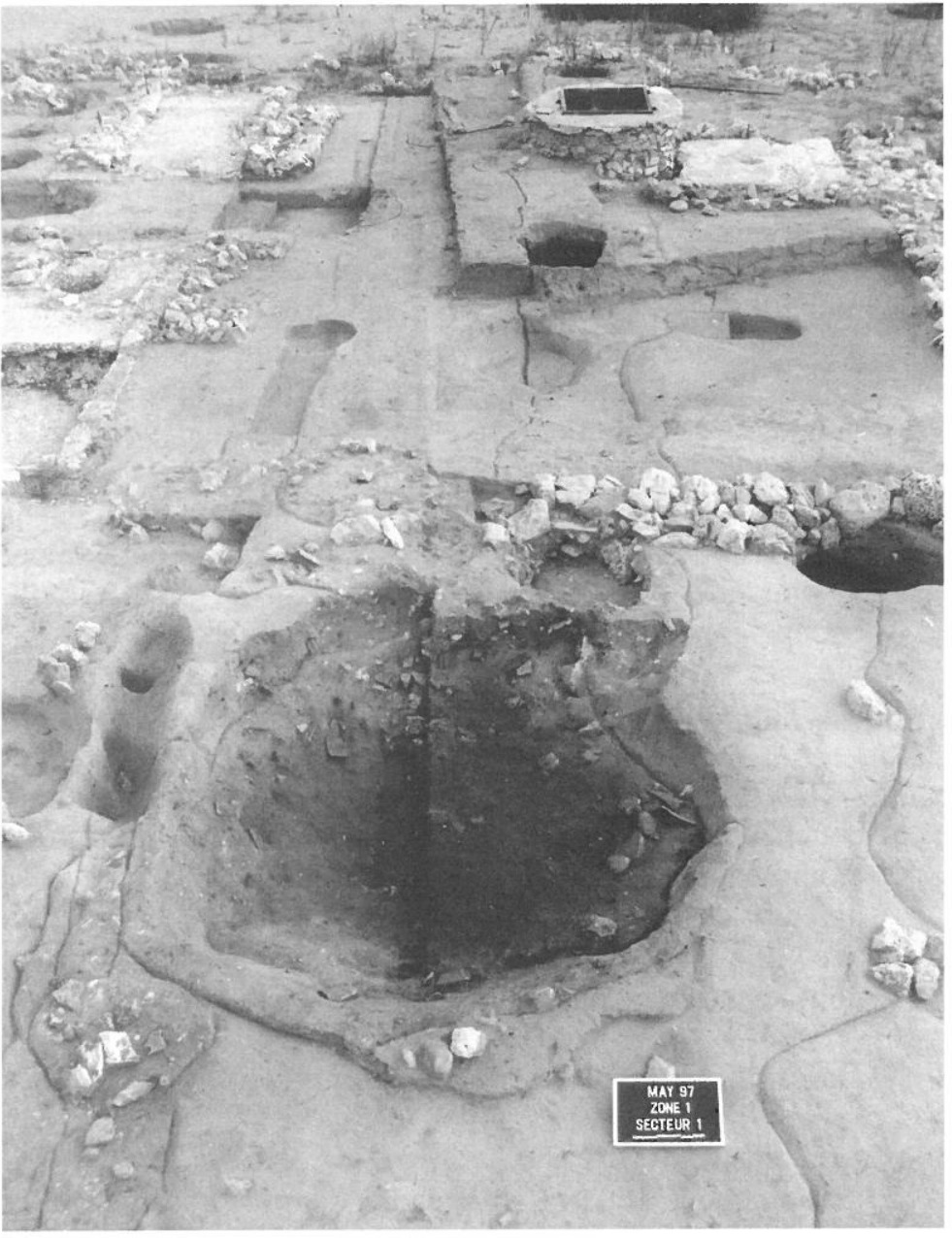

Fig. 48. La couche 1220 comblant une dépression sur la tranchée de l'aqueduc (cliché L. Buffat)

s'être progressivement tassé. Elle recouvre une fine couche sableuse (1219), vraisemblablement contemporaine (recollages entre les tessons des deux niveaux). Elle est recoupée, au début du Moyen Âge, par la construction d'un four (1042).

À l'instar du niveau 1001, la couche 1220 est constituée principalement d'un sédiment charbonneux de teinte noirâtre. Les scories qu'elle contient (plus de $80 \mathrm{~kg}$ ) n'appellent aucune remarque particulière. Elles sont identiques à celles de la couche 1001 (gangues alvéolées en calotte portant parfois une empreinte de tuyère). La couche inclut par contre d'épaisses plaques de substrat (sable pliocène) fortement rubéfiées et indurées par le feu. Ces agglomérats correspondent manifestement à des fragments de parois ou de sole d'un foyer, rejetés ici après un curage. Quelques plaques présentent une face externe vitrifiée qui témoigne de l'intensité de la chauffe au sein du foyer.

Céramiques et verrerie découvertes dans ces deux couches situent sans conteste ces deux ensembles dans le courant du $\mathrm{V}^{\mathrm{e}} \mathrm{s}$. Les termini sont par contre difficiles à préciser. Ce sont les céramiques communes qui apportent le plus d'informations. Parmi les productions kaolinitiques, on retrouve les prototypes de marmites qui ne semblent se développer qu'à partir du dernier tiers du $\mathrm{V}^{\mathrm{e}} \mathrm{s}$. (forme KAOL B32 notamment, Raynaud in Py 1993, 495). Cette poterie occupe une place encore secondaire au sein de ce lot, alors qu'elle est majoritaire dès les années 470 sur le site voisin de Lombren à Vénéjan (CATHMA 1993, 116123). Même si le mobilier des deux couches entretient des parentés typologiques avec cet ensemble, il semble donc qu'il faille le situer plus tôt, vraisemblablement durant le deuxième tiers du $\mathrm{V}^{\mathrm{e}} \mathrm{s}$.

\section{INVENTAIRE DU MOBILIER}

1001 (425/475) phase C4 - luisante: 8 fr. (Lamb. 45), claire africaine: 3 fr. (Hayes 73, 64/80 A), lampe à huile: 2 fr., claire engobée oxydante : 2 fr. (CL-ENG B11, Rigoir 18a), commune à pisolithes oxydante: 18 fr. (PISO A11, B5c, C2b, C2c [2]), oxydante micacée : 8 fr. (COM-E-M A3 var.), kaolinitique réductrice: 9 fr. (KAOL A22, B30 var., B32 var. [2]), amphore hispanique: 11 fr. (1 anse Dr. 23, 6 panses Dr. 23, 4 panses probablement lusitaniennes), amphore africaine : $4 \mathrm{fr}$., céramique indéterminée: $1 \mathrm{fr}$.

Verre: 9 fr. de couleur olive dont 3 gobelets Isings 106C et une coupelle Isings 116

Fer: 2 fr. de clous, 1 objet indéterminé, 1 possible pêne de serrure, 1 fragment présentant une extrémité biseautée (martelage?), 2 informes

Bronze: 1 plaque de bronze percée, 1 bracelet.

1220 (425/475) phase C4 - luisante: 4 fr. (indét.), estampée grise : $2 \mathrm{fr}$. ( Rigoir 2 à décor de rouelles), claire récente : 1 fr., claire engobée oxydante : 4 fr. (CL-ENG B11), kaolinitique oxydante: 2 fr. (KAOL C8), kaolinitique réductrice : 5 fr. (KAOL A22), oxydante micacée: 4 fr. (1 urne de type inédit), pisolithes oxydante: $14 \mathrm{fr}$. (PISO A9, B5f), amphore africaine: 1 fr., amphore orientale: 1 fr. (anse LRA 4), amphore indéterminée: $1 \mathrm{fr}$.

Verre: 2 fragments de couleur olive

Fer: 4 fragments de clous, 1 objet indéterminé (mors?), 1 possible clé, 1 plaque de métal informe

Bronze: 1 fragment d'anneau (diam.: environ $3 \mathrm{~cm}$ )

\section{Interprétation et comparaisons}

Les scories de Mayran laissent peu de doute quant à l'activité métallurgique qui était pratiquée au sein de la pars fructuaria. Elles proviennent indubitablement d'une forge (d'après l'examen de V. Serneels). On manque malheureusement d'exemples de forges domaniales pour pouvoir dépasser ce premier stade d'interprétation. Le petit complexe dégagé non loin de Mayran, sur la villa de la Ramière à Roquemaure constitue le seul parallèle disponible en haut Gard rhodanien (Maufras, Fabre 1998). Cette forge, datée du V ${ }^{\mathrm{e}} \mathrm{s}$., est installée dans une pièce de 
l'ancienne parte résidentielle. Elle comporte une grande pierre plate, peut-être une enclume, et un foyer en fosse de $0,78 \times 0,38$ $\mathrm{m}$. Les parois de ce foyer, que nous avons pu examiner lors de la fouille, présentent de fortes similitudes avec les plaques de substrat rubéfiées retrouvées dans la couche 1220 : teinte ocre, rubéfaction des bords sur une épaisseur d'environ $5 \mathrm{~cm}$. Cette similitude nous conduit naturellement à restituer un ou des foyers similaires sur le gisement de Mayran. L'hypothèse paraît d'autant plus recevable que ce type de four est couramment attesté dans la région depuis l'âge du Fer.

Évaluer la production de la forge de Mayran n'est pas chose facile. La fouille n'a dégagé que la frange orientale d'un complexe

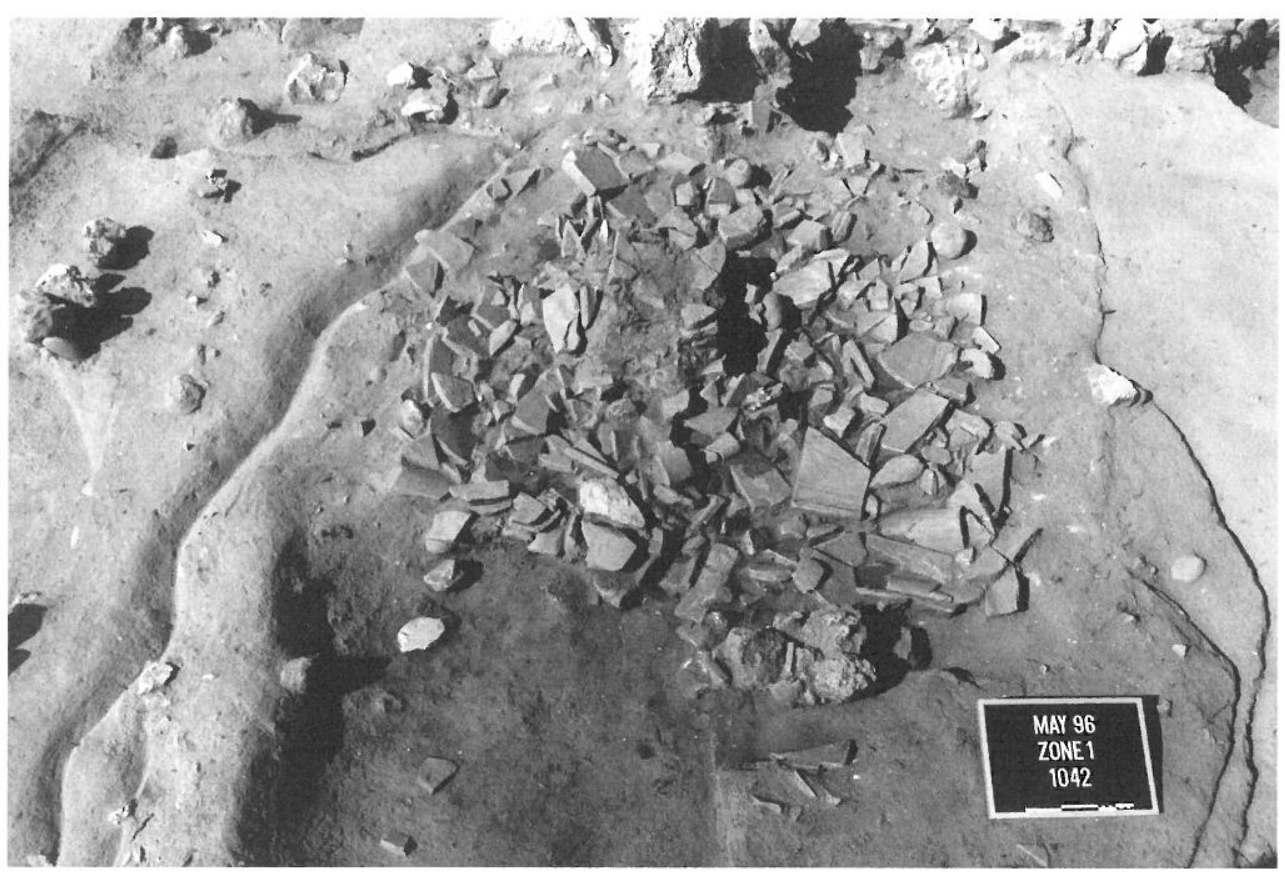

Fig. 49. Le four 1042 sur son radier de tuiles (cliché L. Buffat). qui, à l'évidence, s'étend au-delà des limites de la fouille. Les prospections menées au contact sud-ouest du chantier le confirment. Elles ont en effet mis en évidence une zone d'environ $100 \mathrm{~m}^{2}$ particulièrement charbonneuse et riche en scories (supra, fig. 35). Il est possible que cette tache cendreuse marque l'emplacement du pôle d'activité métallurgique, à moins qu'il ne faille la localiser plus à l'ouest, au niveau d'une seconde concentration de scories, repérée en prospection.

Une dernière remarque, qui concerne la densité des scories, peut être formulée. En effet, il apparaît clairement que les couches 1001 et 1220 , chronologiquement proches, se sont formées rapidement. Une production minimale de plusieurs centaines de $\mathrm{kg}$ de scories sur quelques décennies, voire quelques années, peut donc raisonnablement être proposée. Â titre de comparaison, la forge de la Ramière ne livre à la même époque qu'une dizaine de kg de scories (Maufras, Fabre 1998). Il est vrai que, dans ce cas, l'urgence de la fouille est susceptible d'avoir faussé les chiffres. N'en reste pas moins que la forge du modeste habitat tardif de la Ramière semble avoir une activité plus limitée que celle du domaine - manifestement plus vivace - de Mayran.

\subsection{Un four très arasé (fig. 47,49 )}

Un four (1042), recoupant un des niveaux de rejet métallurgique (fig. 47), constitue un des aménagements romains les plus tardifs repérés sur le site. Il est fondé sur un radier de tegulae et d'imbrices (1223) de forme sub-

rectangulaire (fig. 49). Sur ce radier est installé un foyer rectangulaire (1224) fait de fragments de tuiles superposés, liés par de l'argile. L'ensemble de la structure prend place sur un remblai préparatoire (1223).

La structure n'a livré aucun élément (graines, scories...) permettant d'identifier sa fonction exacte. Étant donné ses dimensions, il est clair qu'il ne s'agit pas d'une structure domestique. Il s'agit plus vraisemblablement d'un four destiné à l'artisanat ou à une fonction agricole, dont la nature exacte nous échappe encore.

\subsection{Une grande excavation (fig. 50)}

Parmi les aménagements tardifs, on compte également une grande fosse repérée au sud-est du chantier. Seule la partie occidentale de cette excavation a été dégagée. Elle se développe de toute évidence vers l'est, au-delà des limites de la fouille. Cette fosse présente un bord en escalier et un fond plus ou moins plat. Elle est accolée à la façade nord du bâtiment à hypocauste. Ce creusement présente un comblement complexe et hétérogène. Les différentes couches observées (us 1215, 1309, 1310, 1311, $1321,1325,1359)$ affectent généralement un pendage vers le centre de la fosse trahissant vraisemblablement un remplissage progressif par "charretées". L'utilisation de cet aménagement n'apparaît pas clairement. On proposera l'hypothèse d'une fosse d'extraction de sable.

Au nord, un niveau de sol, de forme rectangulaire a été aménagé sur le comblement de la fosse. Il est marqué par une fine couche de tuiles pilées (1214). Il semble qu'il 


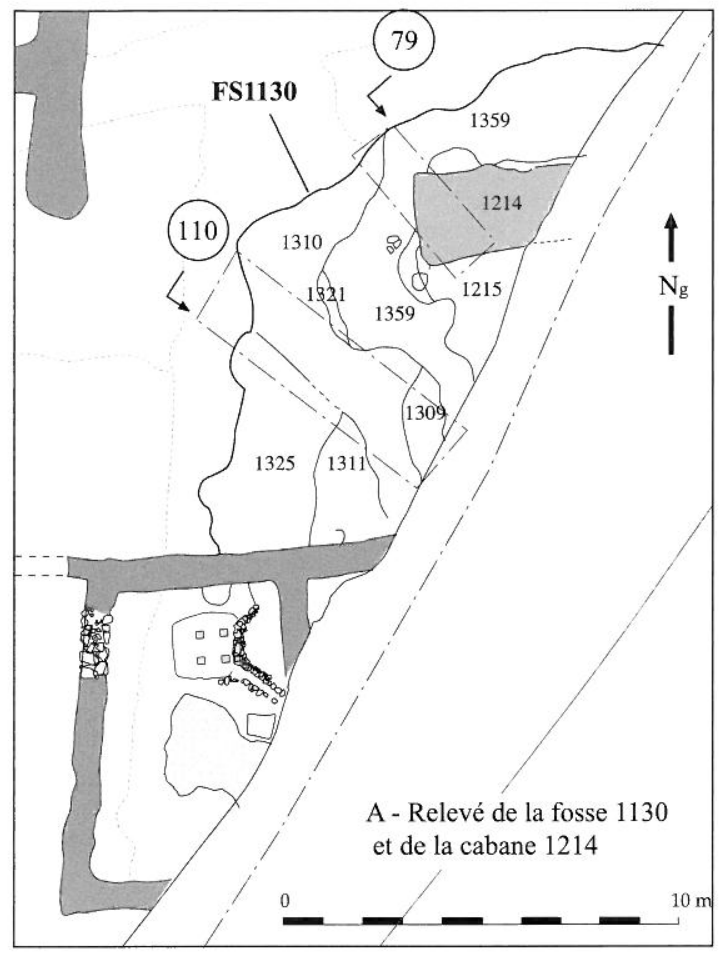

Fig. 50. Plan et coupes de la fosse 1130 et de la cabane 1214.

La fosse 1130 , particulièrement importante, se développe au-delà des limites de la fouille. La cabane 1214, marquée par un niveau de sol et des calages de poteau, prend place sur le comblement de cette grande excavation.

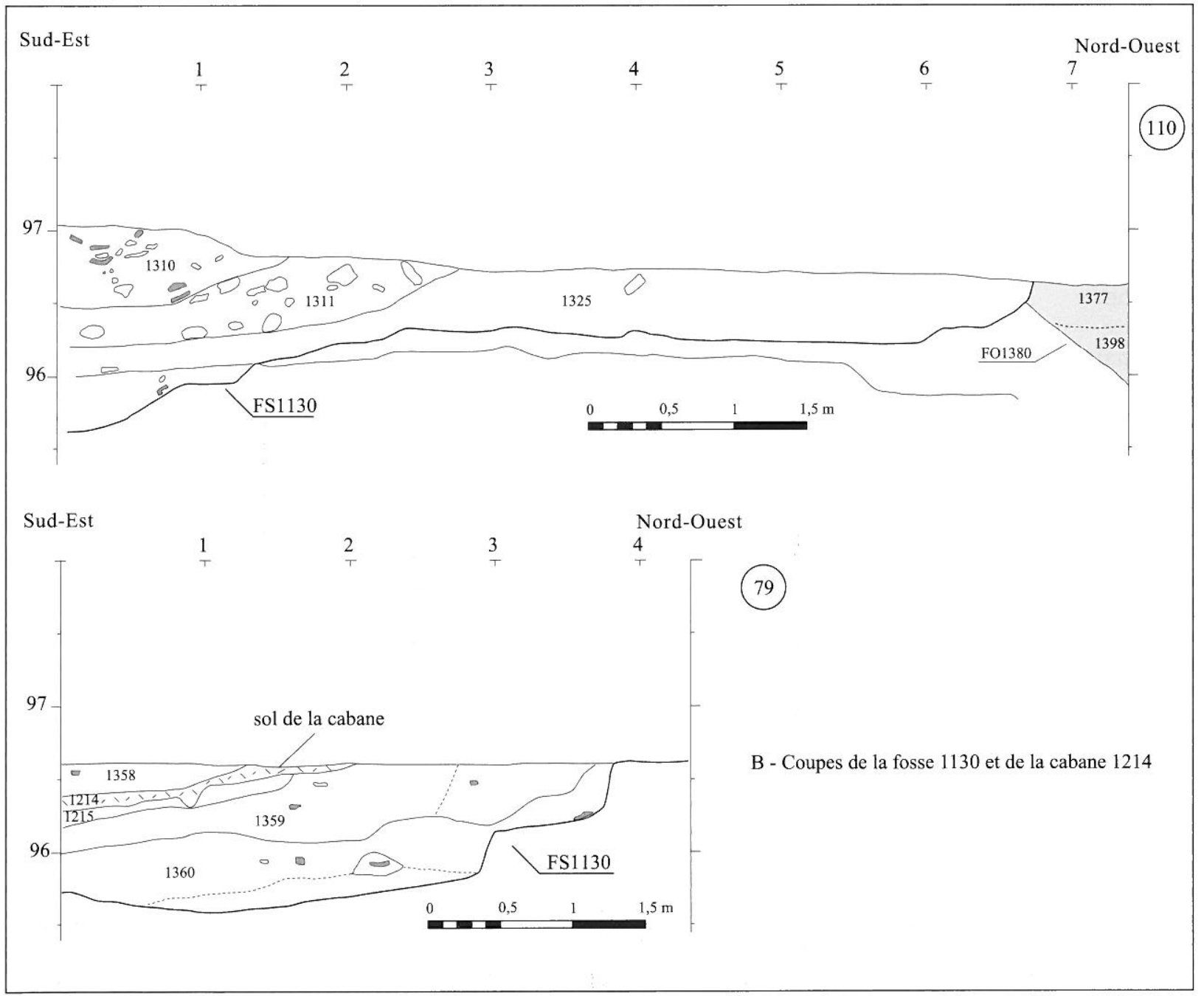


s'agisse du sol d'une petite cabane construite en matériaux légers. Deux trous de poteau, repérés à l'ouest, sont apparemment les traces de la superstructure de cette petite cabane. Aucun élément ne permet de proposer une interprétation sur la fonction de cet édicule. Quelques scories collectées dans le niveau d'abandon pourraient mettre cet espace en relation avec le travail métallurgique, mais il s'agit d'une hypothèse bien fragile.

\section{Datation}

Le mobilier abondant recueilli dans cette excavation situe son comblement dans la première moitié du $\mathrm{V}^{\mathrm{e}} \mathrm{s}$. Le mobilier indique également que la cabane installée sur les niveaux de remplissage est mise en place peu après le comblement de la fosse. La couche qui scelle son abandon livre des éléments céramiques qui diffèrent peu de ceux retrouvés dans la fosse. On situera donc le fonctionnement de cet édicule dans la même plage chronologique, peutêtre durant le second quart du $\mathrm{V}^{\mathrm{e}} \mathrm{s}$.

\section{INVENTAIRE DU MOBILIER}

\section{Comblement de la fosse 1130}

1215 (400/425) phase C4 - claire B/luisante: 10 fr. (Portout 2, 37a, 62), lampe africaine: 1 fr., estampée orangée: 1 (Rigoir 28), claire engobée oxydante : 5 fr. (plat indét., coupe indét.), claire récente: 2 fr., oxydante micacée: 9 fr. (COM-E-M A2, B2), kaol. oxydante: 7 fr. (KAOL B6, indét.), amphore gauloise: 1 fr., amphore hispanique: 1 fr., amphore africaine : 2 fr., amphore orientale: $1 \mathrm{fr}$. Total céramiques: $40 \mathrm{fr}$.

1309 (375/425) phase C4 - claire B/luisante: 1 fr., oxydante micacée: 1 fr., kaol. oxydante: 1 (KAOL B24b). Total communes: 2 fr. Amphore de Bétique: 2 fr., amphore africaine: 1 fr. (retaillé en bouchon). Total amphores: 3 fr. Total céramiques: $6 \mathrm{fr}$.

Huîtres : 2 ; peson retaillé dans une tuile : 1 ; tuile surcuite : 2

1311 (400/425) phase C4 - claire B/luisante: 11 fr. (Portout 1, 12, 37a), claire $C: 1$ fr., claire $D: 1$ fr. (Hayes 64), claire engobée oxydante: 14 fr. (CL-ENG B11), claire récente : 2 fr., oxydante micacée: 14 fr. (COM-E-M A3, cruche indéterminée, COM-OM B3), kaolinitique oxydante: 35 fr. (KAOL $\mathrm{B} 22, \mathrm{~B} 24, \mathrm{C} 8$, indét., couvercle indét.), kaolinitique réductrice : 9 fr. (KAOL A24, B31, C7, cruche indéterminée), pisolithes oxydante: 9 fr. (PISO B5a, B5b), commune indéterminée: 1 fr., amphore gauloise sabl.: 2 fr., amphore de Bétique: 18 fr. (Dr23, Dr23A), amphore hispanique: 14 fr., amphore africaine: 27 fr. (2 Keay 25, 2 fonds indét.), amphore orientale: 7 fr., amphore indéterminée: $10 \mathrm{fr}$. Total céramiques: $175 \mathrm{fr}$.

Bronze indéterminé : 1 fr.; huîtres: 20 ; clou en fer: 3 ; 1 fr. de substrat vitrifié

Monnaie: 1 petit bronze de Constance II (346-354?); os animaux : $41 \mathrm{fr}$; aiguille en os : 1 ; tubulure micacée : 1 fr.; verre olivâtre: 15 fr. (1 bord coupé, 1 décor ondé, 3 décors d'oves noirs); verre transparent: 1 fr.

$1316(400 / 450)$ - claire B/luisante: 1 fr. (Portout 37a), claire engobée oxydante: 1 fr., claire récente : 2 fr., kaolinitique oxydante: 1 (KAOL A3), kaolinitique réductrice: 9 fr. (KAOL A24), amphore de Bétique: 1 fr., amphore africaine: $1 \mathrm{fr}$., amphore indéterminée: $1 \mathrm{fr}$. Total céramiques: $17 \mathrm{fr}$.

Os animaux : 9 fr.; tubulure micacée: $1 \mathrm{fr}$.

$1325(375 / 425)$ phase $C 3$ - claire B/luisante: 1 fr., lampe à huile à pâte calcaire: 1 fr., lampe africaine: 1 fr., claire engobée oxydante: 6 fr., claire récente: 2 fr., oxydante micacée: 3 fr. (COM-E-M C3), kaolinitique oxydante: 7 fr. (KAOL A17, A20, indét.), kaolinitique réductrice: 2 fr., pisolithes oxydante: 3 fr., commune indéterminée: 1 fr., amphore gauloise sabl.: 1 fr., amphore de Bétique: 2 fr., amphore hispanique: 2 fr., amphore africaine: 3 fr., amphore indéterminée: 1 fr. Total céramiques : 36

Scorie de fer: 2 fr.; os animaux : $19 \mathrm{fr}$.

1310 (400/425) phase C3 - claire B/luisante: 7 fr. (Portout 3), estampée grise: 1 fr., claire engobée oxydante: 8 fr., claire récente : 2 fr., oxydante micacée : 7 fr. (urne indét.), kaol. oxydante: 19 fr., kaol. réductrice: 3 fr. (KAOL A20, B24), pisolithes oxydante: $4 \mathrm{fr}$. (PISOC2c), commune indéterminée: 1 fr., amphore de Bétique: 9 fr., amphore hispanique: 3 fr., amphore africaine: 12 fr., amphore indéterminée: 5 fr. Total céramiques: $81 \mathrm{fr}$.

1359 (375/425) phase C3 - claire B/luisante: 1 fr., claire D: 1 fr. (Hayes 59, indét.), claire récente: 2 fr., kaolinitique oxydante: 3 fr., kaolinitique réductrice: $1 \mathrm{fr}$. (KAOL C8), pisolithes oxydante: 4 fr. (PISO B5a, B5f, C2c), amphore africaine: 1 fr., amphore indéterminée: $1 \mathrm{fr}$. Total céramiques: $15 \mathrm{fr}$.

Huîtres: 2; clou en fer: 1; monnaie: 1, moyen bronze de Gallienus (253-268); os animaux : 5

1360 phase C3 - kaol. réductrice: 8 fr. (4 bords indéterminés) os animaux : 5

Abandon de la cabane

1358 (425/475) phase C4 - campanienne A : 1 fr., claire B/luisante: 3 fr., claire D : 1 fr., claire engobée oxydante: 1 fr., oxydante micacée : 3 fr. (bord triangulaire inédit), kaol. oxydante: 4 fr., kaol. réductrice: 14 fr. (KAOLA20, B24a), piso. oxydante: 4 fr. (PISO C2c), amphore gauloise sabl.: 2 fr., amphore hispanique : 1 fr., amphore africaine : 3 fr., amphore indéterminée: $3 \mathrm{fr}$. Total céramiques : $40 \mathrm{fr}$.

Coquillage indéterminé : 1 , scorie de fer: 9 , os animaux : 35 fr.

\section{L'OCCUPATION MÉDIÉVALE}

La fouille de Mayran a mis en évidence une importante et durable occupation médiévale. Celle-ci se manifeste majoritairement par des fosses d'ensilage (70 fouillées) dont la chronologie couvre une période très large qui va $\mathrm{du} \mathrm{VI}^{\mathrm{e}}$ aux $\mathrm{XII}^{\mathrm{e}}-\mathrm{XIII}{ }^{\mathrm{e}} \mathrm{s}$. L'intérêt vient ici du fait que la plupart des fosses ayant été fouillées, il est possible de détailler sur 8 siècles l'évolution des formes et de la densité de l'ensilage. L'absence totale de stratification demeure par contre un handicap pour cerner précisément les chronologies, la céramique médiévale étant généralement difficile à dater précisément. Ceci nous a conduits souvent à proposer des datations assez larges, couvrant un siècle, voire deux. L'absence totale de vestiges d'habitat reste également une lacune de taille. L'arasement pro- 


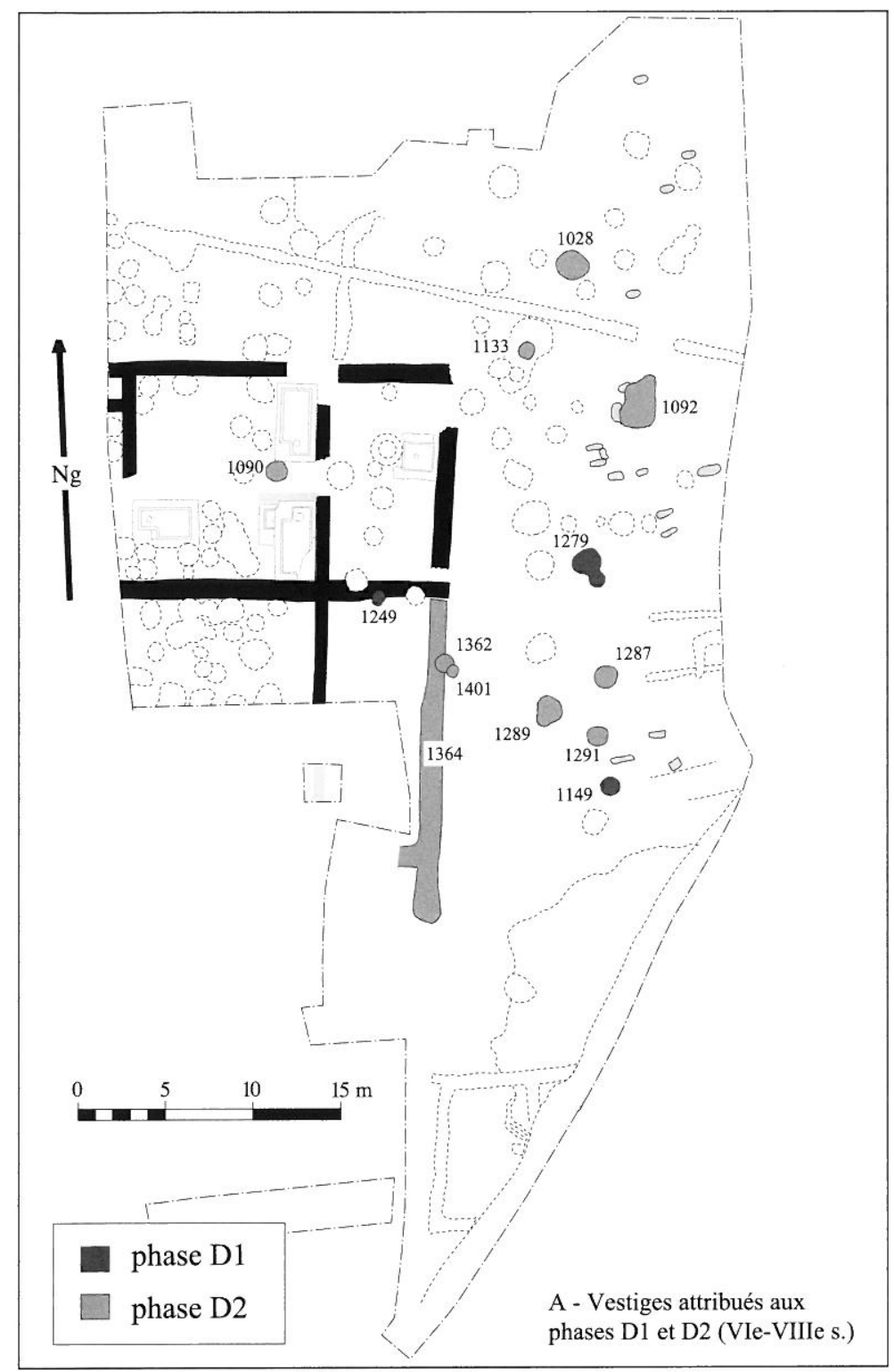

Fig. 51. Les aménagements du début du Moyen Âge: phase D1 ( $\left.\right|^{e} \mathrm{~s}$.), phase D2 (VIle-première moitié VIIIe s.).

Les aménagements de cette période correspondent pour l'essentiel à des fosses et silos. On suppose qu'une partie des murs du bâtiment antique sont restés visibles, mais la mauvaise conservation des vestiges ne permet d'en être certain.

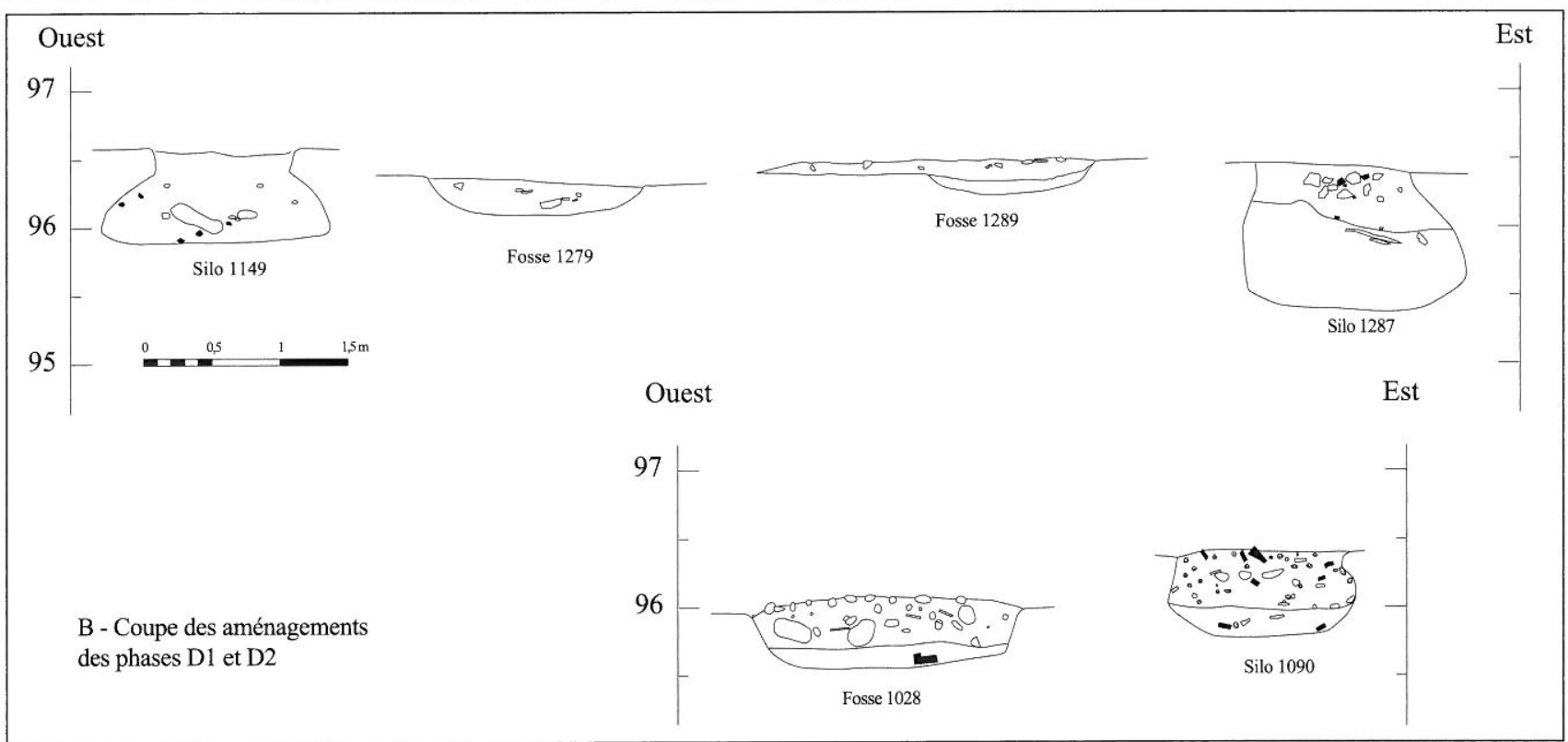



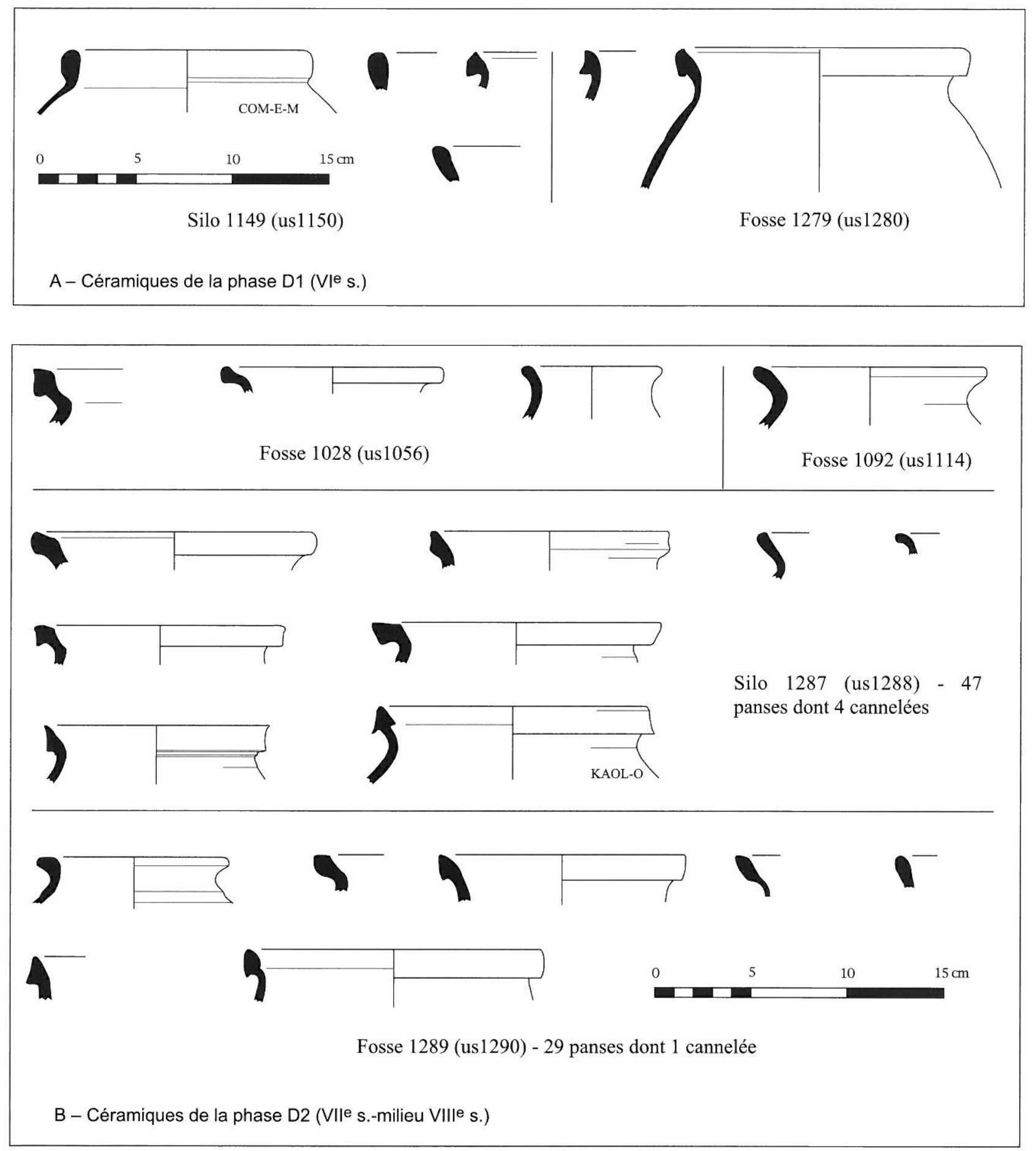

Fig. 52. Céramiques des $\mathrm{Vle}-\mathrm{VIII}{ }^{\mathrm{e}} \mathrm{s}$.

L'essentiel des productions de cette période correspond à de la céramique kaolinitique réductrice provenant de la région d'Uzès. Sauf indication contraire, toutes les céramiques sont kaolinitiques à cuisson réductrice.

noncé du site est peut-être responsable de cette absence. Mais comment avoir quelque certitude à ce sujet? Il faudra renoncer à mettre en relation les structures de stockage avec l'habitat.
Les nombreuses fosses fouillées apportent des informations intéressantes sur la vaisselle utilisée à cette période, grâce à un nombre important de contextes (89 ensembles représentant 3138 tessons). Exclusivement 


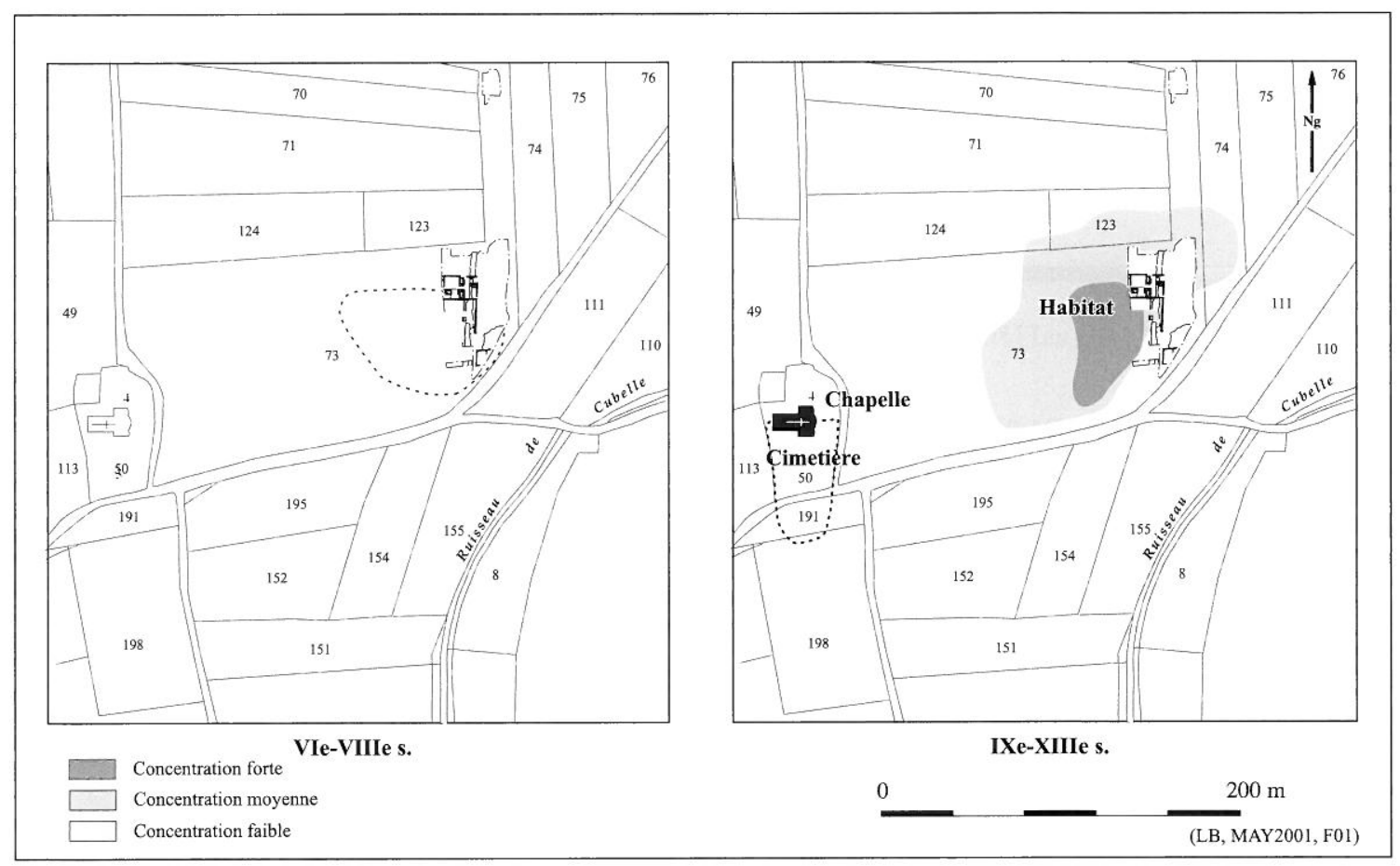

Fig. 53. L'évolution du site de Mayran durant le haut Moyen Âge, d'après les prospections de surface. Un recul de l'occupation est sensible à partir du VIe s. Dans le courant du VIIIe $\mathrm{s}$. ou le début du IX ${ }^{\mathrm{e}} \mathrm{s}$., les indices se multiplient. La chapelle est datée du XIIe $\mathrm{s}$. On ignore si elle succède à un établissement antérieur.

kaolinitique, la céramique témoigne d'une évolution lente. Il serait cependant hors de propos d'étudier en détail ces céramiques. Nous n'en présenterons ici que quelques planches de synthèses.

\subsection{L'occupation du début du Moyen Âge (phases D1 et D2, $\mathrm{VI}^{\mathrm{e}}-\mathrm{VIII}^{\mathrm{e}} \mathrm{s}$. fig. 51 à 53)}

Les fouilles, comme les prospections montrent une réduction de l'occupation à partir $\mathrm{du} \mathrm{VI}^{\mathrm{e}} \mathrm{s}$. On ne trouve pour la période comprise entre le $\mathrm{VI}^{\mathrm{e}}$ et le $\mathrm{VIII}^{\mathrm{e}} \mathrm{s}$. que quelques rares silos et fosses. La prospection atteste elle aussi ce fort étiage de l'occupation, qui se concentre dans un secteur restreint de l'ancienne villa.

\subsubsection{Des vestiges ténus (fig. 51)}

L'abandon de la partie agricole de la villa de Mayran se situe assez tardivement, si l'on en croit la découverte d'un fond d'amphore africaine de la fin du Ve ou du VIe s. dans une cuve. Les structures vinicoles pourraient toujours être utilisées, du moins entre les années 450-550.

\section{- Le VI ${ }^{\mathrm{e}} \mathrm{s}$. (phase D1)}

Globalement, le $\mathrm{VI}^{\mathrm{e}} \mathrm{s}$. correspond dans la partie fouillée à une période de tassement de l'occupation. Hormis la cuve que nous venons d'évoquer, seules deux fosses et un silo témoignent d'une continuité de l'occupa- tion. Ces aménagements restent assez anecdotiques. Le silo 1149 occupe une position isolée au sein de l'ancien vignoble antique. Il constitue la plus ancienne trace d'ensilage repérée sur le site. La fosse 1249 présentait au sommet de son comblement des galets rubéfiés et de blocs calcaires éclatés sous l'action du feu. Il est possible que cette fosse corresponde à un foyer. Le cas de la fosse 1279 est problématique En marge de toute structure contemporaine, elle présente un profil régulier qui induit un usage spécifique. Morphologiquement, elle s'apparente aux silos "semi-enterrés" de Lunel-Viel (Raynaud 1990, 80, 82). $\mathrm{Cl}$. Raynaud considère, en se basant sur les travaux de F. Sigaut (1978), que ce type de fosse pouvait être destiné « à la conservation d'autres produits que les grains, par exemple, les betteraves, pommes de terre ou certains fruits, ainsi protégés du gel, ou encore pour la fermentation de plantes fourragères ou du chou ». La découverte de fragments de couvercle en lauzes dans le comblement de la fosse plaide en faveur de cette hypothèse. Nous retiendrons donc l'interprétation, sans pouvoir préciser la nature du produit stocké.

\section{- Les VII'-VIII ${ }^{\mathrm{e}}$ s. (phase D2)}

Pour la période comprise entre le VII ${ }^{e}$ et le VIII ${ }^{e}$ siècle (phase D2), les indices d'occupation ne sont guère plus nombreux que pour le $\mathrm{VI}^{\mathrm{e}} \mathrm{s}$. (fig. 51). L'ensilage souterrain, attesté dès le $\mathrm{VI}^{\mathrm{e}} \mathrm{s}$., se développe dans la périphérie de l'établissement gallo-romain, et de façon ponctuelle 


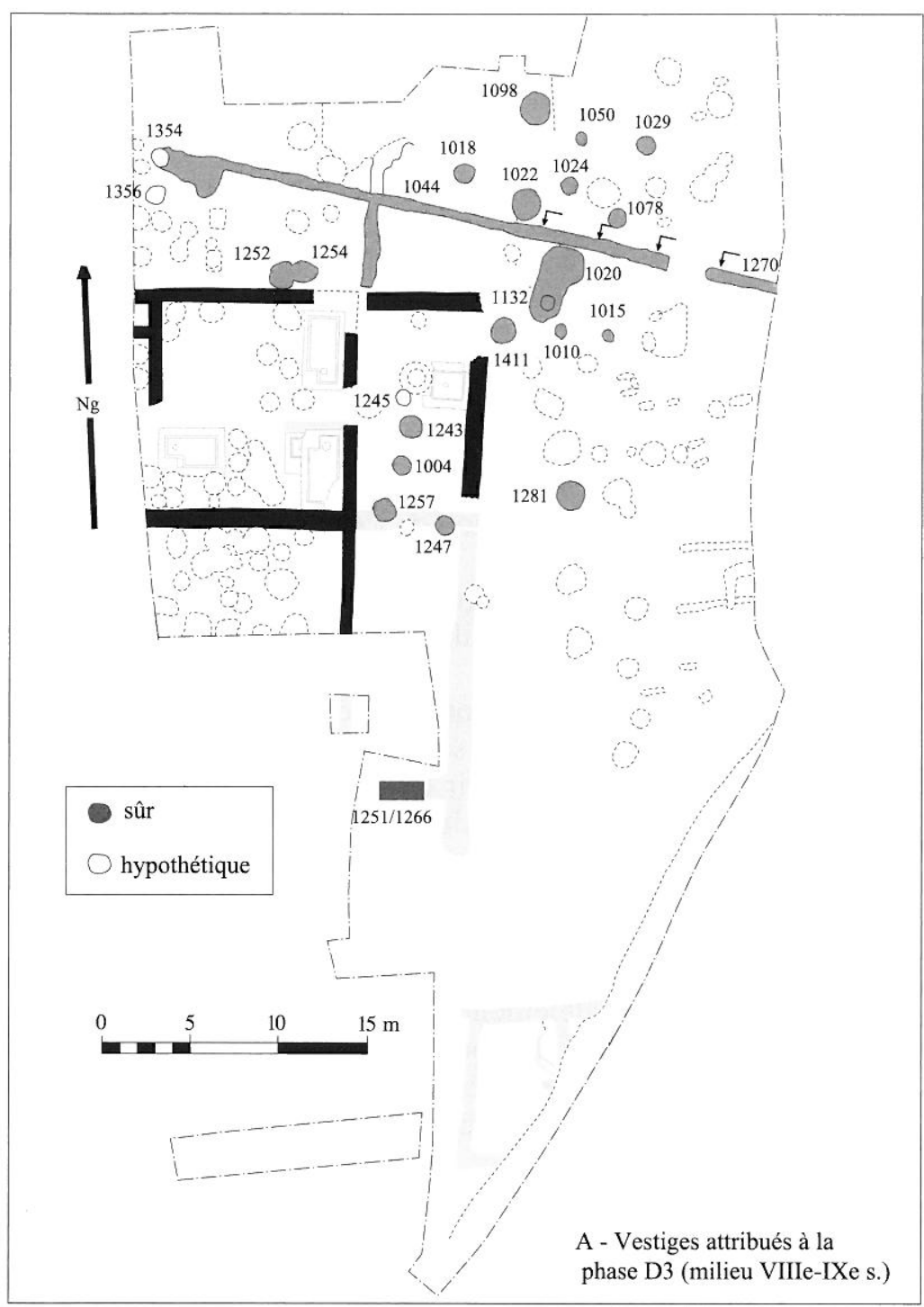

Fig. 54. Les aménagements datés du début de la période carolingienne (seconde moitié du VIII $\mathrm{s}$., IX $\mathrm{s}$.).

Les vestiges correspondent pour l'essentiel à des silos. Une partie des murs du bâtiment romain semble toujours en élévation. Un fossé est-ouest structure ce pan de paysage. On trouve des limites parcellaires modernes isoclines avec cette orientation. phase D3 (milieu VIIIe-IXe s.)

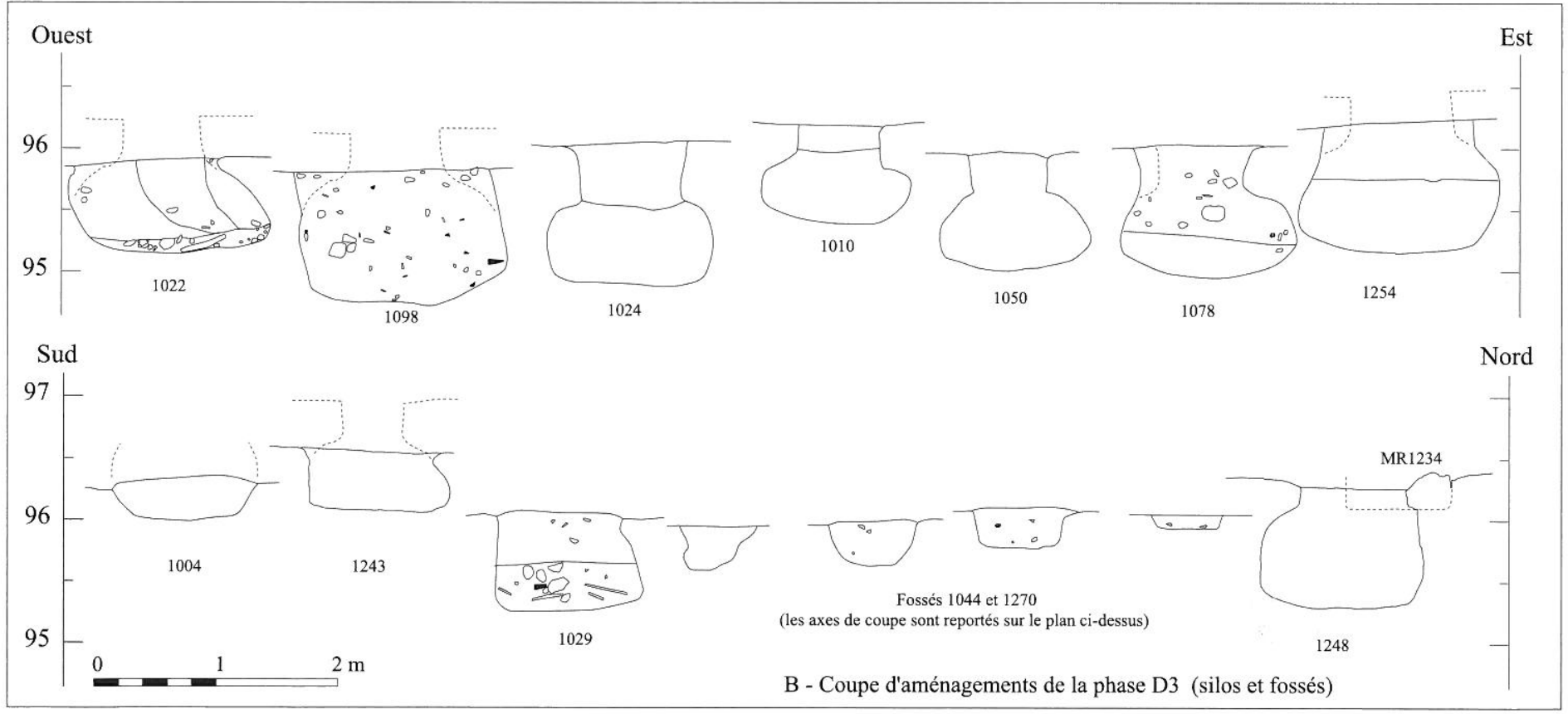




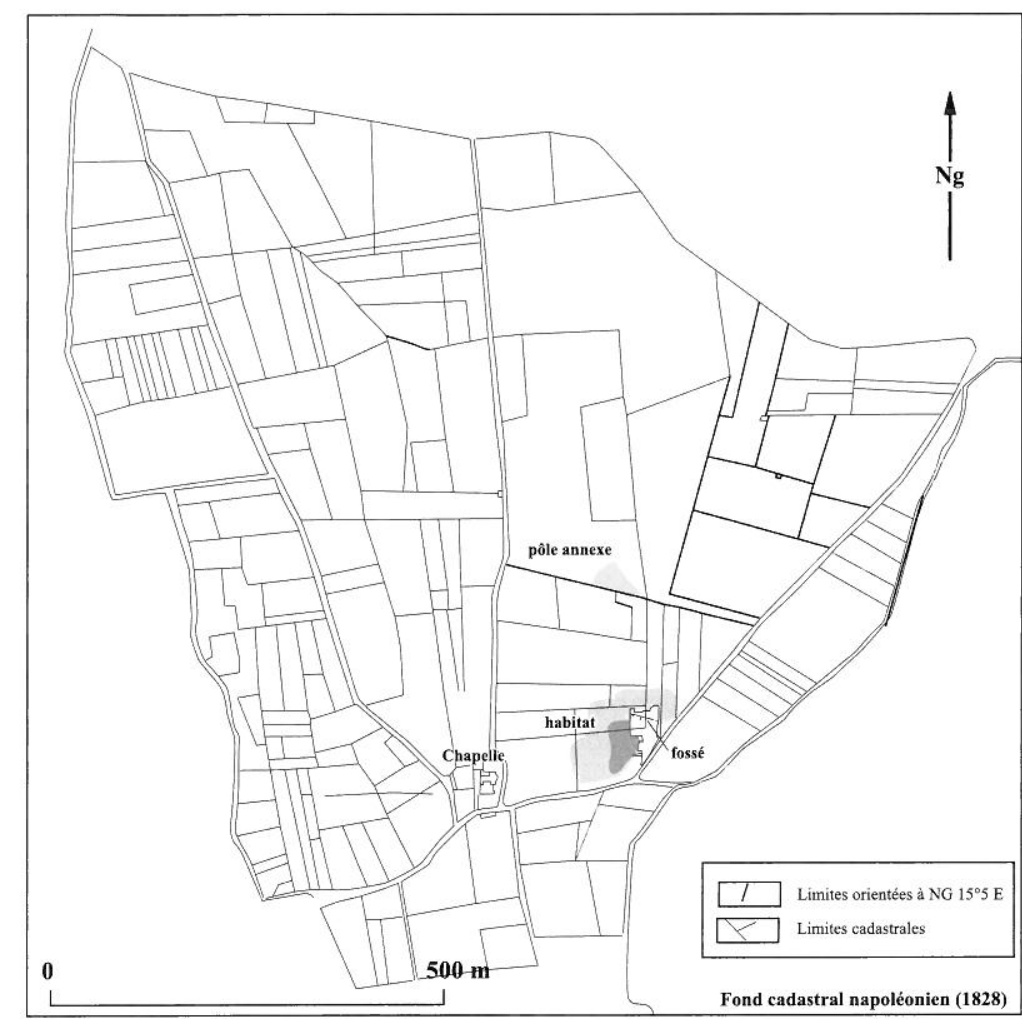

Fig. 55. Limites parcellaires modernes isoclines avec le fossé carolingien: les traces d'un parcellaire médiéval?

dans l'emprise de celui-ci. À cette période, on assiste aux premiers chantiers de récupération de matériaux. Le mur sud-est du bâtiment antique est épierré sur toute sa longueur (1364). Un silo s'installe dans l'ancienne cella vinaria, dont les cuves sont désormais comblées. Pour autant, les murs qui délimitaient cette pièce semblent toujours debout. En tout cas, ils ne présentent aucune trace d'épierrement.

L'aire d'ensilage qui se développe à l'est des bâtiments romains est modeste: quelques silos et fosses en sont les rares témoignages. Ces différents aménagements n'ont certainement pas fonctionné simultanément. Seules exceptions peut-être, la fosse 1289 et les silos 1291, 1287 qui forment un groupe cohérent, tant topographiquement que chronologiquement.

Si par leur forme, les silos 1090 et 1287 ne posent pas de problème d'identification, il n'en va pas de même pour les fosses 1028, 1092 et 1289. Concernant la fosse 1028, on se trouve dans un cas de figure proche de celui de la fosse 1279. Le creusement, soigné, évoque une utilisation précise. Par analogie, on la rangera dans la catégorie des silos semi-enterrés, mais cette attribution reste sujette à caution. Il paraît plus hasardeux d'interpréter les deux autres aménagements en l'absence d'indices probants.

\section{Image d'ensemble du site de Mayran aux VI ${ }^{e_{-}}$} $V_{\text {VIII }}{ }^{s}$. : données de prospection (fig. 53)

En prospection de surface, la diminution de la surface occupée et du nombre d'artefacts est très marquée à partir du $\mathrm{VI}^{\mathrm{e}} \mathrm{s}$. Les témoins qui se rattachent au début du Moyen Âge , pour l'essentiel des bords triangulaires en céramique kaolinitique réductrice (type KAOL A29/CATHMA 6a, 6c) ou des bords en poulie (CATHMA 7b), sont peu nombreux et localisés dans une zone réduite couvrant environ 0,4 ha. Les données de la prospection rejoignent celles de la fouille pour témoigner d'une rétraction de l'occupation.

\subsection{L'ensilage durant la période carolingienne} (phase D3, milieu VIIIe s. - IX ${ }^{\mathrm{e}}$ s.) (fig. 54-56)

Après l'atonie du début du Moyen Âge (VI ${ }^{\text {- }}$ VIII ${ }^{\mathrm{e}} \mathrm{s}$.), le site témoigne d'une activité plus intense à partir de la fin du $\mathrm{VIII}^{\mathrm{e}} \mathrm{s}$. ou du $\mathrm{IX}^{\mathrm{e}} \mathrm{s}$. Deux aires d'ensilage vont se développer. Un grand fossé structure ce pan de paysage. Celui-ci présente une orientation particulière, isocline à des limites parcellaires actuelles. Ce pourrait être l'indice d'un parcellaire d'époque carolingienne. Parallèlement, les prospections révèlent une reprise de l'occupation, caractérisée par une augmentation de la surface occupée et du nombre d'indices d'occupation.
Fig. 56. Céramiques kaolinitiques réductrices du début de la période carolingienne trouvées dans les silos de la phase D3a (deuxième moitié du VIII' $s$. - IX $\mathrm{e}$ s.).

L'influence antique a définitivement disparu dans ce répertoire, qui se compose exclusivement d'urnes globulaires, dont le diamètre d'ouverture n'excède jamais $13 \mathrm{~cm}$. Les décors, jusqu'alors rares, se développent de façon sensible. Les cannelures horizontales en constituent la grande majorité. Elles caractérisent de façon efficace ce faciès, même si leur apparition est légèrement antérieure (supra, phase D2).

J. Kotarba observe le même phénomène sur le site d'Augéry de Corrèges, à côté d'Arles: «la proportion de panses cannelées par rapport aux autres panses est toujours assez forte (entre 18 et $37 \%$ ). Elle paraît plus importante dans les produits kaolinitiques et pour les niveaux les plus anciens " (Kotarba in Cathma 1993, 145). La datation retenue pour le faciès ancien de ce gisement est le $\mathrm{IX}^{\mathrm{e}} \mathrm{s}$., avec cependant une tendance plus marquée pour la première moitié de ce siècle (Ibid.).

Les décors à la molette sont présents, mais semblent essentiellement rattachables aux productions les plus récentes. La typologie est dominée par les bords Cathma 5, à marli droit ou incliné. 

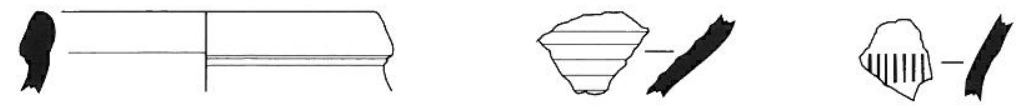

Fig. 56

Silo 1004 (us1002) : 6 panses dont 1 cannelée et 1 décorée à la molette
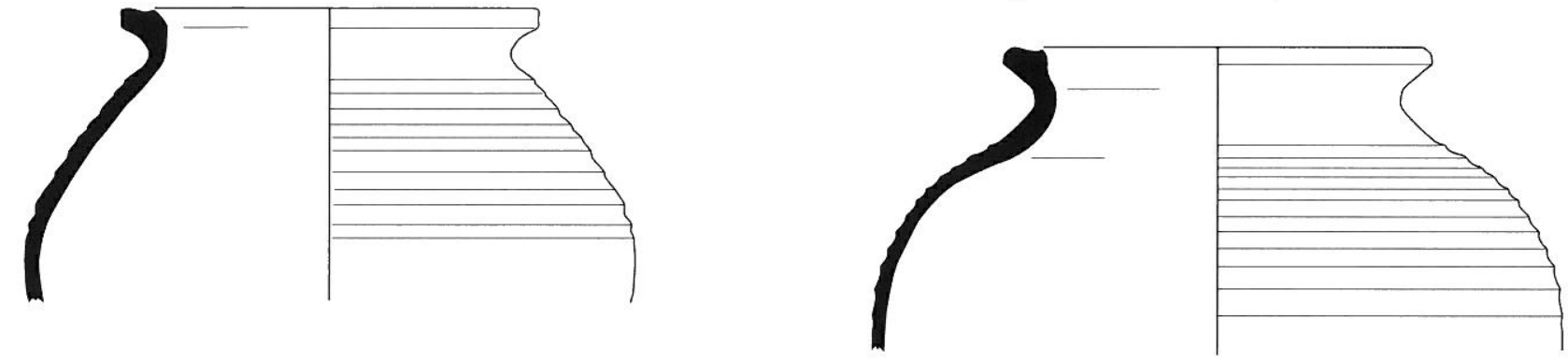

Silo 1022 (us 1057) : 90 panses dont 32 cannelées

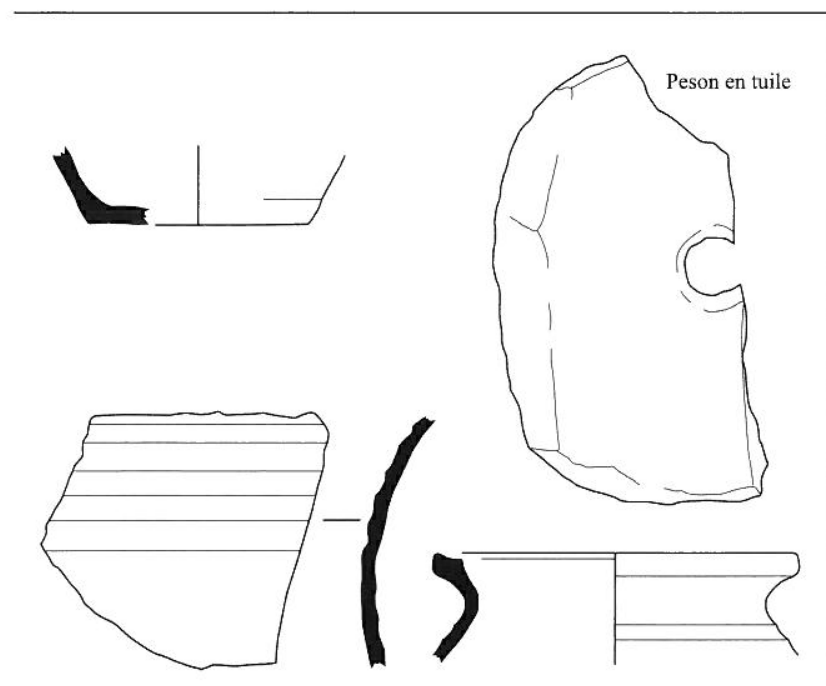

Silo 1010 (us 1011) : 26 panses dont 13 cannelées

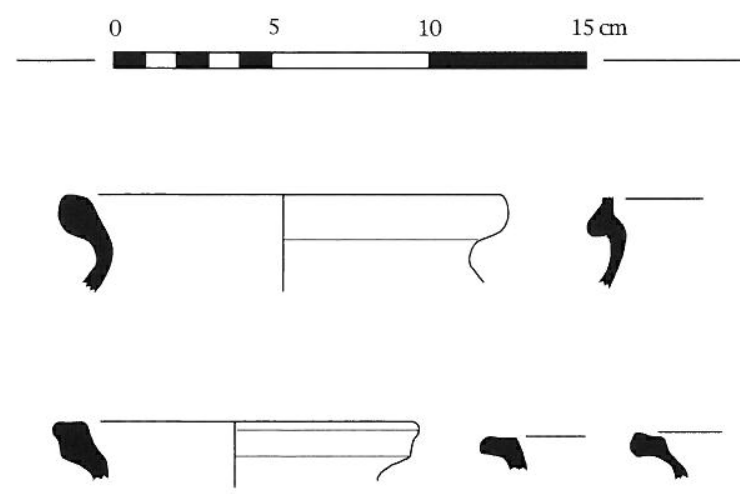

Silo 1098 (us1099) : 67 panses dont 31 cannelées et 3 décorées à la molette

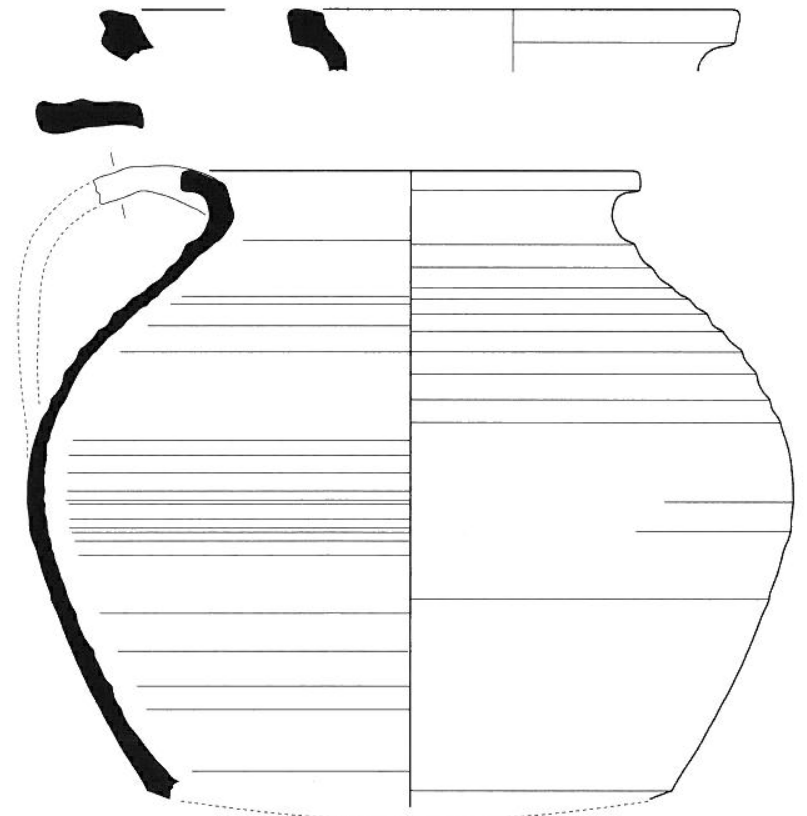

Silo 1247 (us1248) : 92 panses dont 49 cannelées

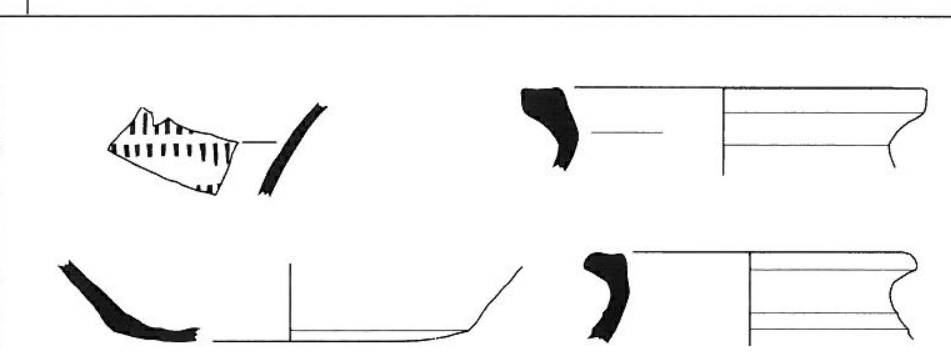

Silo 1018 (us1111) : 12 panses dont 3 cannelées et 1 décorée à la molette 
Fig. 57. Les vestiges attribués la phase D4 (fin IX $\left.X \mathrm{I}^{\mathrm{e}} \mathrm{s}.\right)$ : implantation des silos dans les bâtiments antiques.

Les silos sont dans leur grande majorité implantés dans la pars agraria romaine. On ne relève pas de cas manifeste de recoupements de constructions antiques. Ceci suggère qu'une partie des murs de la villa pouvaient être toujours utilisés.
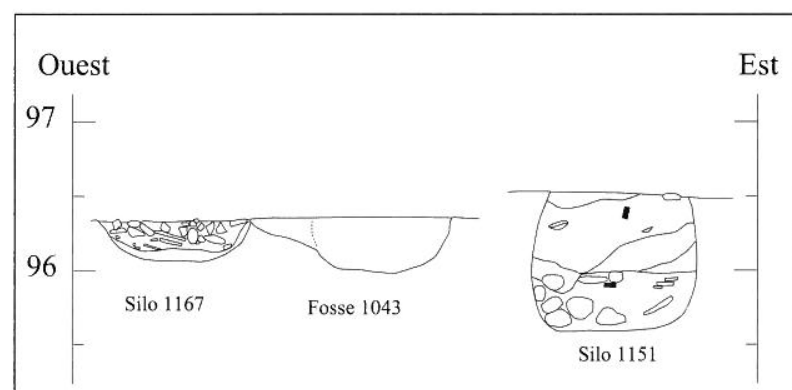

Ouest

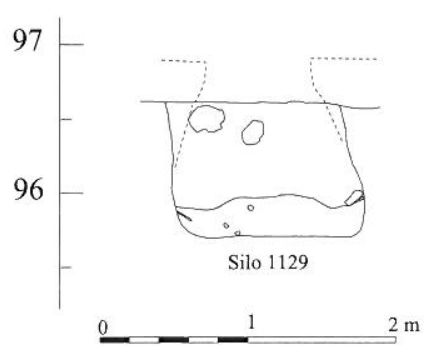

Sud
$97+$
$96-$
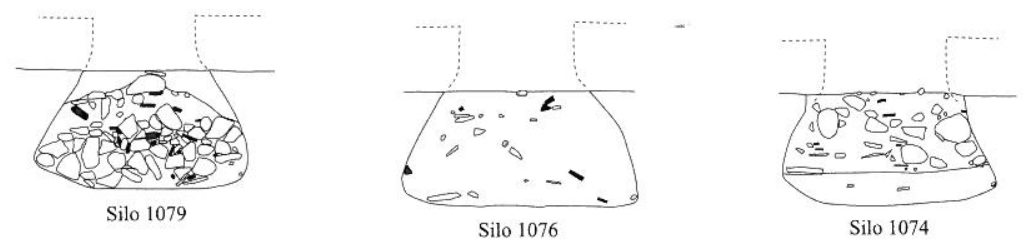

B- Coupes des silos de la période D4
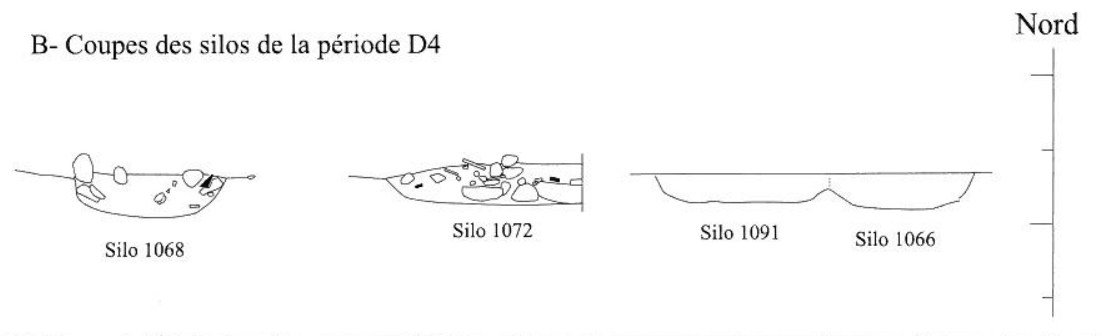

A - Plan des vestiges attribués à la phase D4 (fin IXe-XIe s.)

Est

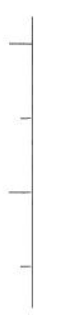

Nord
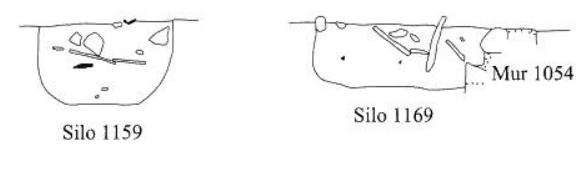

Silo 1068 


\subsubsection{Les aires d'ensilage (fig. 54)}

La première aire d'ensilage, au nord-est, comprend une douzaine de silos. Elle se développe de part et d'autre d'un fossé qui traverse la fouille d'est en ouest. Cette aire d'ensilage présente une organisation régulière. Les fosses à grains sont implantées à intervalles réguliers. On ne peut évidemment affirmer qu'elles ont fonctionné simultanément, mais l'absence de recoupements entre les structures témoigne certainement d'une gestion raisonnée et probablement continue de l'espace. Le fossé 1044/1270 est un élément majeur dans cette aire de stockage. Il comporte à l'est une interruption, probablement un passage permettant la communication entre les deux parcelles. L'examen de son profil (en U, fig. 54 ) révèle une "usure" qui trahit vraisemblablement une longue utilisation et un entretien régulier. On pourrait supposer qu'il servait à préserver la zone de l'humidité, en drainant les eaux de ruissellement, mais en l'absence d'exutoire ou de fosse de déversement, cette hypothèse reste difficile à soutenir. S'agit-il surtout d'une limite parcellaire? L'attraction qu'il exerce sur les silos n'est de toute façon pas anodine.

Une seconde aire d'ensilage se développe dans la partie orientale des ruines de la villa. Elle comporte cinq silos. Ceux qui se trouvent au nord sont fortement arasés et il n'en subsiste que le fond. Les silos prennent place dans un secteur où les fondations de la villa antique n'ont pas été récupérées. Deux silos (1247 et 1257) recoupent le mur situé au sud, qui devait alors être hors d'usage. Ce n'était visiblement pas le cas des autres murs qui ont pu être réutilisés, peut-être pour aménager une couverture audessus des silos. Au nord-ouest du chantier, de rares silos pourraient compléter ce dispositif de stockage, mais leur datation est incertaine ( ils n'ont livré que quelques tessons).

Les silos d'époque carolingienne présentent une morphologie assez caractéristique. C'est le profil en "ampoule" qui domine $(1010,1024,1029,1078)$. L'examen des profils témoigne du soin apporter au creusement de ces fosses. Une ouverture étroite (diamètre variant de 0,6 à $0,8 \mathrm{~m}$ ) donne accès à une chambre de stockage à parois plus ou moins galbées, avec fond plat ou légèrement convexe. La largeur de ces chambres varie assez peu (entre 1,2 et 1,6 m). Des cas d'effondrement de la cheminée ont été relevés $(1029,1098,1254)$ comme sur de nombreuses aires d'ensilage (Raynaud 1990, 80, fig. 46).

\subsubsection{Les traces d'un parcellaire carolingien?}

La présence d'un long fossé rectiligne d'époque carolingienne (1044/1270) invite également à s'interroger sur la morphologie agraire à cette période. Peut-on mettre en relation cette structure avec d'autres éléments? L'analyse du fond cadastral de 1828 (fig. 55) révèle que plusieurs limites parcellaires présentent une orientation similaire
(NG $15^{\circ} 5^{\prime} \mathrm{E}$ ). Peu nombreuses, elles sont localisées dans un espace bien circonscrit, au nord-est du site. S'agit-il des vestiges d'un système parcellaire carolingien? Il est tentant de le croire, même si les éléments de confirmation archéologique sont encore insuffisants pour le prouver. Outre l'isoclinité de ces limites avec le fossé dégagé lors de la fouille, il faut noter qu'une concentration de mobilier des $\mathrm{IX}^{\mathrm{e}}-\mathrm{XII}{ }^{\mathrm{e}} \mathrm{s}$. est limitée au sud par une séparation de parcelles répondant à cette orientation.

Les certitudes sont minces mais ces structures posent un certain nombre de questions sur l'organisation du paysage à l'époque carolingienne. Cet exemple bien modeste invite en tout cas à poursuivre le travail sur la morphologie agraire durant le haut Moyen Âge, dont on sait désormais qu'elle ne se résume pas à des parcellaires lanièrés ou radio-concentriques (Abbé 1998, 225). De la même façon, l'exemple de Mayran met en garde sur l'interprétation et la datation, parfois abusives, des parcellaires orthonormés.

\subsubsection{Le site à l'époque carolingienne et au Moyen Âge médian $\left(I X^{e}-X I I I^{e} s.\right)$ : l'apport des prospections}

Il reste difficile, à partir des données de la prospection, de discerner l'évolution globale du site entre le IX ${ }^{\mathrm{e}}$ et le $\mathrm{XIII}^{\mathrm{e}} \mathrm{s}$. Les bords de céramiques ou les décors collectés en surface ne sont pas suffisamment caractéristiques pour distinguer une évolution au cours de cette longue période. Nous adoptons donc ici un parti prudent en envisageant globalement cette phase de l'occupation du site.

L'occupation se maintient à cette époque à l'emplacement de la villa du Bas-Empire (fig. 53). Si l'on s'en tient aux informations livrées par les tests de ramassage, il faut restituer une zone de forte concentration couvrant 0,3 ha. On trouve des indices, mais en quantité plus faible, sur une surface d'1 ha environ. À cette période, la mise en place de la chapelle et le développement du cimetière qui lui est associé constitue un événement majeur. Mais il est difficile de situer la période d'apparition de la chapelle. L'acte de 896, précédemment évoqué, n'en parle pas, ce qui ne signifie évidemment pas que la chapelle n'existait pas. L'édifice actuel est daté du XII ${ }^{\mathrm{e}} \mathrm{s}$. Quoi qu'il en soit, il semble que l'édifice religieux n'attire pas à lui l'habitat, qui semble centré sur les ruines de la villa (là où se trouve la plus forte concentration de mobilier). Il semble bien y avoir une bipolarisation du site, où sont clairement dissociés le pôle religio-funéraire et le pôle d'habitat. En cela, le site rejoint d'autres exemples régionaux, comme La Gramière à Castillon-du-Gard (Buffat 2004, 265-267).

\subsection{Une aire de stockage dans les ruines du bâtiment} antique (phase D4, entre la fin du IX et la fin du IX ${ }^{\mathrm{e}} \mathrm{s}$.

C'est dans la villa antique que va se développer l'ensilage durant le $\mathrm{X}^{\mathrm{e}} \mathrm{s}$. La pièce où se se situaient les cuves de 


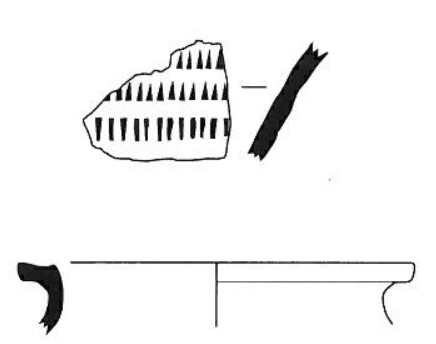

Silo 1078 (us1084) : 18 panses dont 3 cannelées et 2 décorées à la molette

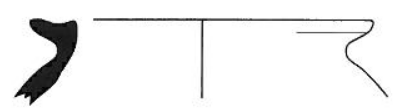

Fig. 58

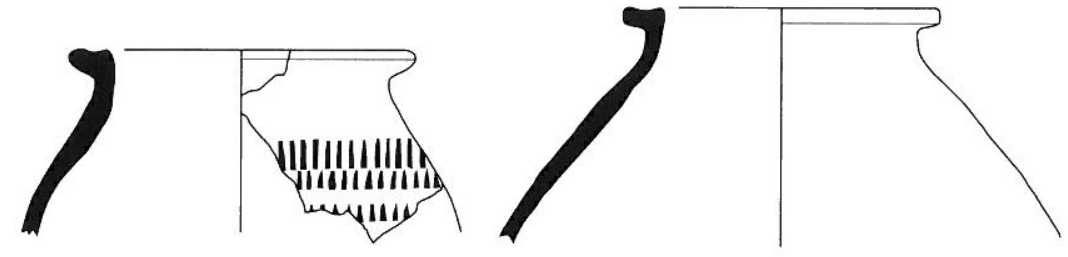

Silo 1068 (us1103) : 99 panses dont 3 cannelées et 14 décorées à la molette
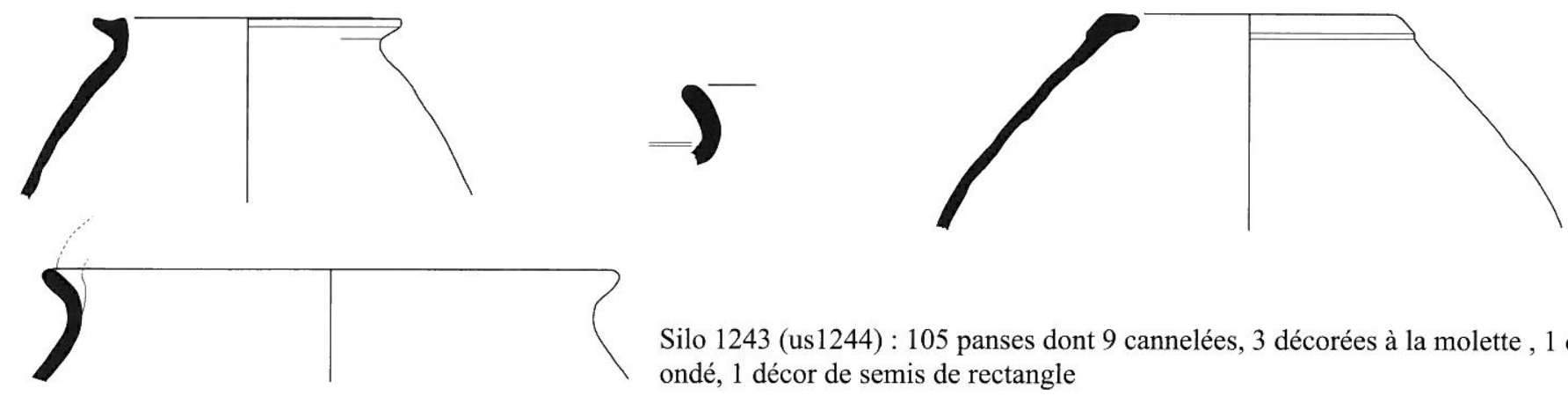

Silo 1243 (us1244) : 105 panses dont 9 cannelées, 3 décorées à la molette, 1 décor ondé, 1 décor de semis de rectangle
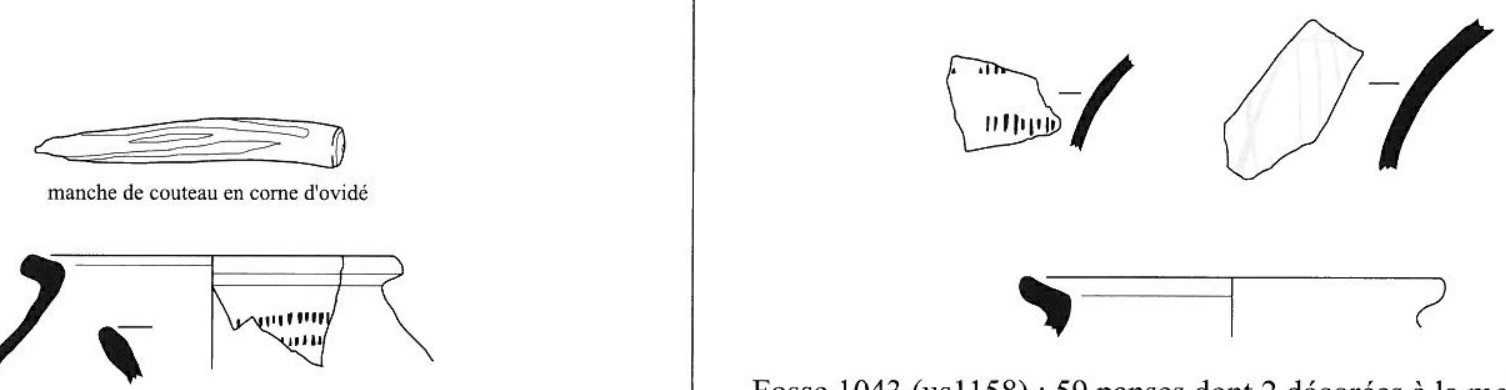

Silo 1075 (us1164) : 33 panses dont 2 cannelées et 1 décorée à la molette

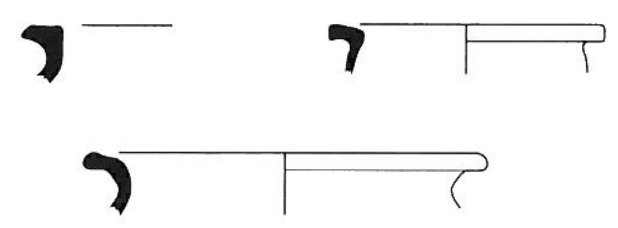

Silo 1068 (us1069) : 12 panses - aucun décor
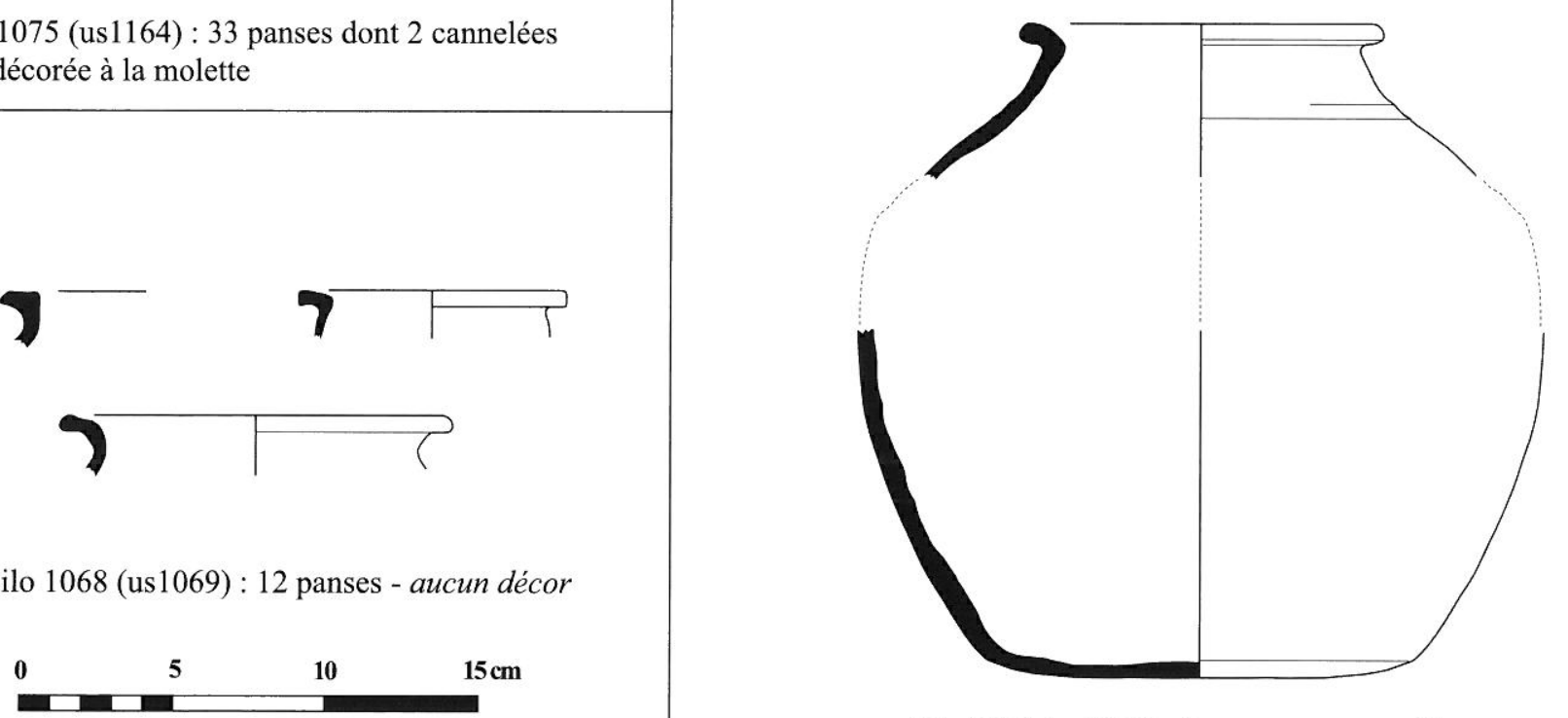

Silo 1066 (us1067) : 3 panses - aucun décor 
décantation est progressivement réinvestie par les silos, qui pour l'essentiel viennent s'accoler contre les architectures romaines.

Le déplacement de l'aire d'ensilage est net durant cette période. On compte encore quelques fosses à l'extérieur de la villa, mais le plus grand nombre prend place dans les ruines de la pars agraria antique. Plusieurs causes peuvent expliquer la réoccupation du bâtiment romain : réutilisation d'architectures pour ménager une couverture aux silos (appentis en matériaux périssables), protection contre le brigandage, volonté de libérer les espaces cultivables. Il reste difficile de retenir l'une ou l'autre des solutions.

L'examen morphologique des fosses n'est pas chose aisée. L'arasement des vestiges est en effet prononcé dans cette partie du site (fig. 57): au nord, les silos 1066, 1068, 1072 et 1091 ne sont plus conservés que sur une vingtaine de centimètres. Quelques exemplaires mieux conservés diffèrent cependant des silos de la phase antérieure (D3) par leur profil en "cloche": fond plat, parois évasées vers le bas.

La répartition des silos est désormais plus serrée qu'auparavant. Il faut dire que le développement de l'aire d'ensilage est limité, contingenté, par l'espace exigu offert par les ruines de la villa. Les recoupements entre les fosses sont fréquents, et ils montrent bien que l'espace disponible est peu important. Par ailleurs, il est probable qu'il existait des constructions antiques, aujourd'hui totalement disparues, qui limitaient plus encore les possibili-

\section{ci-contre}

Fig. 58. Les céramiques de la phase D4 (entre la fin du IXe et la fin du XIe $\mathrm{S}$.).

Les éléments font encore défaut pour identifier très clairement les critères propres à ce faciès. La difficulté tient à la faiblesse numérique des lots et à l'atypisme des formes. Les ensembles de cette époque font cependant figure de "charnière" entre les productions du IXe $\mathrm{s}$. et celles du XII $\mathrm{s}$. La disparition progressive des cannelures constitue la principale évolution par rapport au faciès antérieur. Les décors sont dominés par les impressions à la molette, registres de bâtonnets apposés sur la partie supérieure des vases (épaule et col). Les bandes obliques obtenues par polissage sont très peu représentées (1 fragment dans la fosse 1043), contrairement au Lunellois où elles sont abondantes dans les contextes contemporains (Cathma 1993, 181). Comme pour le faciès antérieur, la typologie est dominée par les bords à marli Cathma 5. Morphologiquement ce type connaît peu d'évolution: un examen attentif révèle une tendance à l'affinement. On note aussi que, sous la lèvre, le col tend à disparaître. Ce faciès trouve peu d'équivalents régionaux. Le contraste est net avec les contextes contemporains du littoral languedocien (Lunellois, bassin de Thau), où dominent les productions grises tendres ou sableuses réductrices, totalement absentes ici (Cathma 1993, 173, 180).

Des ensembles proches sont néanmoins connus à la Commanderie de Sainte Luce, en Arles (Ibid., 140-144). Les catégories représentées sur ce site sont différentes, mais les parentés typologiques sont fortes: domination du type Cathma 5 , présence de becs pontés, abondance des décors à la molette (Ibid., 142, fig. 14). tés de creusement des fosses, notamment dans la pièce des cuves (pièce 1) où pouvait exister une grande construction (plateforme de pressurage?) dans un espace vierge de tout silo (supra, 4-4).

\subsection{Les dernières fosses d'ensilage (phase D5, entre le $\mathrm{XI}^{\mathrm{e}}$ et le $\mathrm{XIII}^{\mathrm{e}} \mathrm{s}$.)}

Les ultimes témoins de présence humaine repérés dans le cadre de la fouille se situent dans les ruines de la villa (fig. 59). Il s'agit là encore de silos, concentrés pour l'essentiel dans la pièce 3 de la pars rustica.

Parmi les silos, apparaît un type nouveau, représenté par les fosses 1153 et 1203. En forme de "sac", il se caractérise par une profondeur importante (dans les deux cas, environ $1,4 \mathrm{~m}$ ). Sa forme évoque une utilisation spécifique, peut-être le stockage en épis. Les autres fosses à grains présentent parfois des évolutions morphologiques. C'est le cas des silos 1126, 11771193 et 1195 qui comportent un fond bombé.

Là encore, l'étude du plan de répartition des silos montre une certaine cohérence dans l'organisation du stockage. Les fosses se développent de part et d'autre du mur 1039. La plus forte concentration se situe au sud. Elle est formée par cinq silos, accolés les uns aux autres (1174, $1177,1179,1181,1193)$. Ce regroupement est intéressant. Il s'oppose en effet à la gestion aérée des aires d'ensilages antérieures. Au nord du mur, aucune logique n'apparait. Les fosses sont toutes localisées au sud de la pièce 1. Elles marquent cependant un déplacement du stockage vers le sud.

\subsection{Les silos médiévaux de Mayran : conclusions}

L'étude des silos ne révèle pas pour l'heure de hiatus ou de période d'abandon de l'aire d'ensilage entre le début du Moyen Âge et le XIII ${ }^{\mathrm{e}}$ s. Il semble que l'on ait affaire à un quartier du site durablement dévolu à cette activité de stockage. Le déplacement progressif des silos est un fait marquant. Dans les premiers temps du Moyen Âge, du VI ${ }^{e}$ au VIII ${ }^{e}$ s., l'aire d'ensilage se développe à l'est du chantier, principalement hors des bâtiments antiques. D'abord limitée à une petite aire au $\mathrm{VII}^{\mathrm{e}}$ et au début du VIII ${ }^{\mathrm{e}}$ s., l'aire se déplace d'une dizaine de mètres vers le nord, dans le courant ou à la fin du VIIIe s., où elle se maintiendra pendant au moins un siècle. $\mathrm{Au} \mathrm{X}^{\mathrm{e}}$ s., un glissement s'opère vers l'ouest. Puis aux $\mathrm{XI}^{\mathrm{e}}-\mathrm{XIII}^{\mathrm{e}} \mathrm{s}$., on perçoit un glissement de l'aire d'ensilage vers le sud, dans le périmètre bâti de la villa romaine. Ainsi, en caricaturant, on pourrait résumer l'évolution de l'ensilage en un mouvement de déplacement circulaire, inverse au sens des aiguilles d'une montre.

On peut s'interroger sur le groupe humain qui utilisait ces réserves de grain. Les silos étaient-ils destinés à un groupe familial? à une petite communauté réunissant plu- 


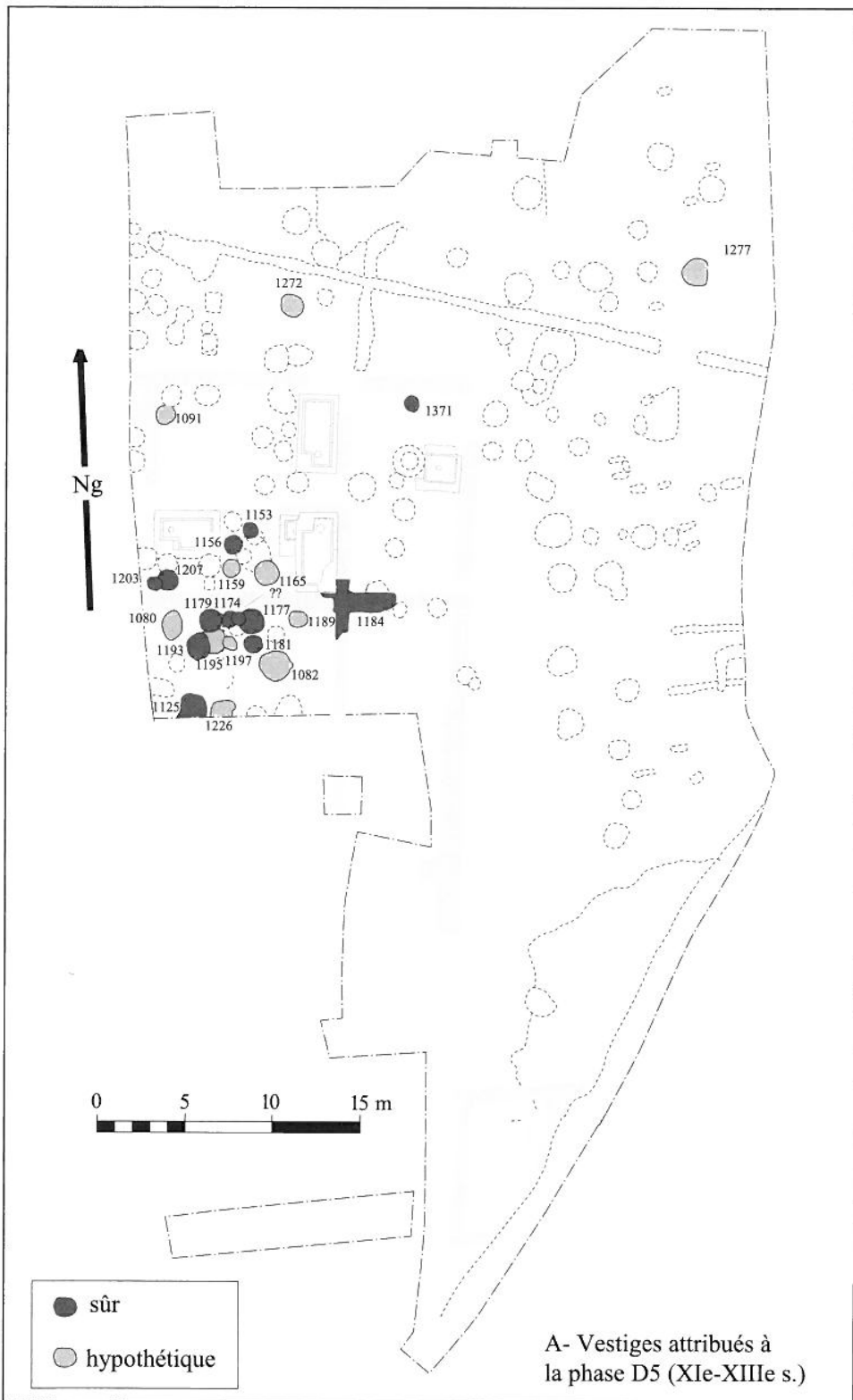

Fig. 59. Les vestiges attribués à la phase D5 (entre le $\mathrm{Xl}^{\mathrm{e}}$ et le XIIIe s.) Les silos sont toujours concentrés dans le bâtiment romain. Les cas de recoupements sont nombreux.

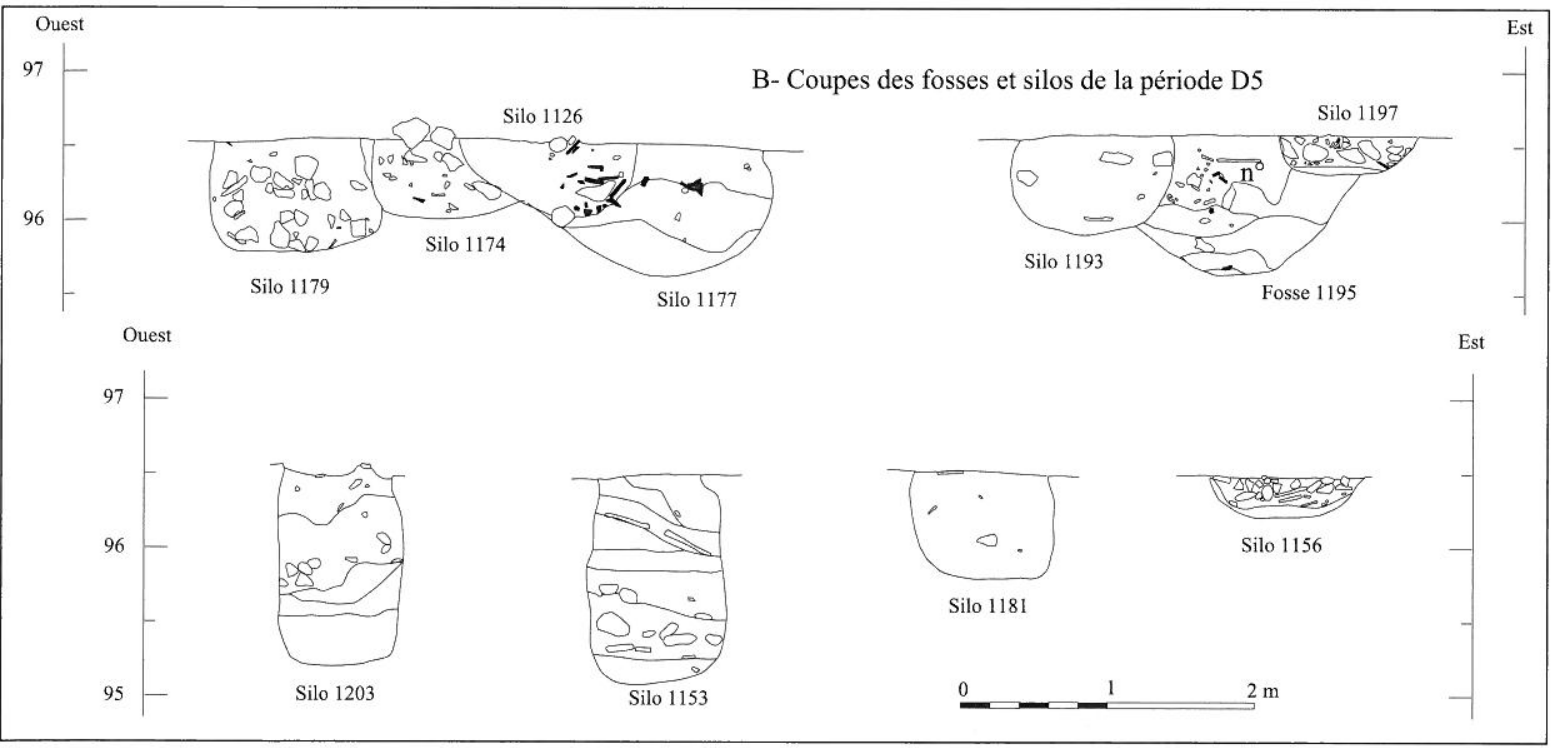




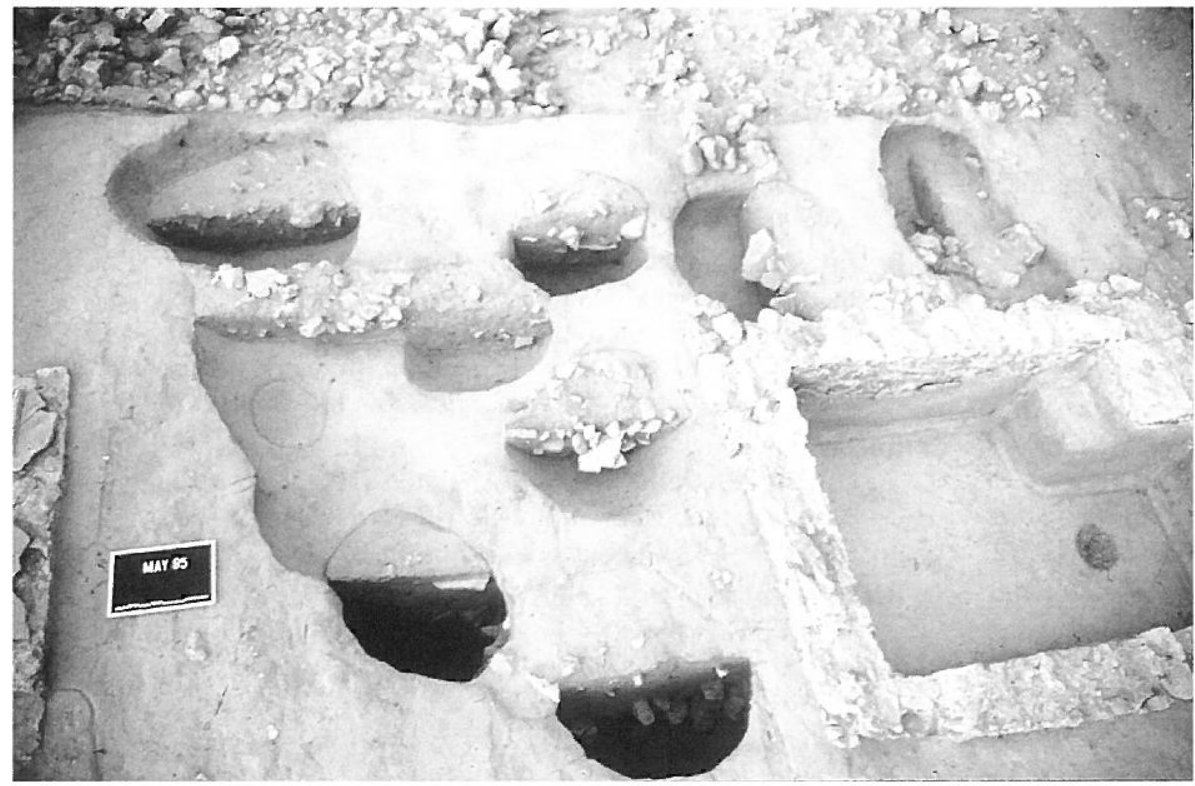

Fig. 60. Silos implantés entre deux cuves romaines (cliché H. Petitot).

Mais peu de choses sont connues de ce premier établissement, dont le cœur se développe en marge de la zone fouillée. Les prospections fines révèlent cependant l'existence d'un pôle central, de près d'un hectare, environné de pôles annexes. En fonction des données actuelles, on estime que le site était déjà une villa à cette époque.

Au Bas-Empire, l'emplacement de la villa s'est sensiblement déplacé. Et c'est cette villa tardive dont la fouille nous a dévoilé certaines caractéristiques. En l'occurrence des bâtiments agricoles, dévolus à la viticulture. Mayran offre ainsi un nouvel exemple d'installation vinicole tardive, qui vient enrichir une série régionale désormais bien étoffée (Buffat, Pellecuer 2001; Buffat 2004). L'exploration de cette villa tardive a également révélé un aqueduc tout à fait remarquable de par son ampleur et sa

sieurs familles? S'agit-il de stocks conservant le fruit d'un prélèvement aristocratique? Il apparaît en premier lieu évident que la situation a dû évoluer durant la longue période qui sépare les plus anciens silos des plus récents (près de 8 siècles). Pour en juger, il faut s'intéresser à la densité des silos tout au long de l'histoire du site. Pour les périodes les plus anciennes, le nombre des fosses apparaît faible. Ainsi, pour l'époque mérovingienne, les fosses sont peu nombreuses et paraissent plutôt correspondre au stock d'une unité familiale. À la période carolingienne, le nombre tend à croître. On doit cependant garder à l'esprit que deux ou trois silos fonctionnant simultanément constituent un grand maximum. Dans cette hypothèse, optimiste si l'on en juge par la fragilité du substrat sableux, les réserves couvriraient les besoins d'un groupe dépassant de peu le cadre familial. L'accroissement du nombre des fosses entre le $\mathrm{X}^{\mathrm{e}}$ et le $\mathrm{XIII}{ }^{\mathrm{e}} \mathrm{s}$. est en revanche perceptible. Cet accroissement s'accompagne d'une installation dans les ruines de la villa. Il reste cependant difficile de tirer des conclusions formelles sur cet accroissement du nombre de fosses. Il serait évidemment tentant d'y voir un accroissement de la population ou bien la création de réserves particulières (fruits d'un prélèvement?).

\section{CONCLUSION}

Mayran est à bien des égards un exemple emblématique des grandes villae qui se sont développées dans la cité de Nîmes. En caricaturant quelque peu, on pourrait dire que ce site résume l'histoire des vastes établissements domaniaux occupés sur le long terme.

Après des débuts timides à l'époque républicaine ( ${ }^{\mathrm{er}} \mathrm{s}$. av. J.-C.), le site prend de l'importance au Haut-Empire. conception. Celui-ci se démarque assez nettement des autres aqueducs domaniaux connus dans la région, qui se présentent le plus souvent comme des ouvrages assez modestes (Pellecuer 2000, 422; Mauné, Paillet 2003, 306, 315-317). L'aqueduc de Mayran a été une réalisation ambitieuse, du moins dans sa partie amont, où il est installé à plusieurs mètres de profondeur. Faute d'avoir pu explorer la totalité de cet ouvrage, on ne peut qu'émettre des hypothèses sur ce dispositif particulier. Nous avons avancé l'idée d'un système de galerie drainante, captant les eaux souterraines du ruisseau voisin. Mais une fouille complémentaire devra valider cette hypothèse.

Durant le Haut Moyen Âge, le site de Mayran est resté un pôle d'habitat important, comme en témoignent les mutiples silos exhumés lors de la fouille, mais aussi les très nombreuses céramiques ramassées en prospection de surface. L'occupation a certes connu un tassement significatif aux $\mathrm{VI}^{\mathrm{e}}-\mathrm{VIII}^{\mathrm{e}} \mathrm{s}$, , mais elle a ensuite repris de la vigueur à la période carolingienne, puis au Moyen Âge central. Sur le plan local, le site de Mayran est donc resté un lieu important et probablement organisateur. On regrettera qu'aucune structure de cet habitat médiéval n'ait été retrouvée. Celles-ci ont probablement disparu sous le coup des charrues modernes, du moins dans la partie fouillée. On reste donc privé d'informations capitales qui nous permettraient d'envisager le passage de la villa antique à la villa médiévale.

Loïc BUFFAT - post-doctorant Lavoisier (ICAC Tarragone) Hervé PETITOT - archéologue INRAP Méditerranée, Nîmes Laurent VIDAL - archéologue INRAP Méditerranée, Nîmes Josseline GUERRE - archéologue contractuelle INRAP Méditerranée Amélie MASBERNAT - place du Moutas, 30700 Sagriès

Élie PÉLAQUIER - CNRS-Université Paul-Valéry Montpellier III 


\section{BIBLIOGRAPHIE}

Abbé 1998 : ABBÉ (J.-L.) - Permanences et mutations des parcellaires médiévaux. In: CHOUQUER (G.) dir. - Les Formes du paysage, Archéologie des parcellaires. Actes du colloque d'Orléans, mars 1996. Paris, Errance, 1998, pp. 223-233.

Andrieu 1995 : ANDRIEU (J.-L.) - Aménagements du territoire et maîtrise de l'eau dans la cité de Béziers du $\mathrm{I}^{\mathrm{er}}$ au Ve $\mathrm{V}$. In: CLAVEL-LÉVÊQUE (M.), PLANA-MALLART (R.) éd. - Cité et territoire. Actes du I ${ }^{\text {er }}$ colloque européen, Béziers (14-16 octobre 1994).Paris, Les Belles Lettres, 1995, pp. 133-147.

Andrieu 1999 : ANDRIEU (J.-L.) - Techniques de construction des aqueducs sur les territoires des cités romaines de Béziers et Narbonne. In: BEDON (R.) éd. - Les aqueducs de la Gaule romaine et des régions voisines. Caesarodunum, XXXI, 1999, pp. 73-108.

Assénat, Pérez 1997 : ASSÉNAT (M.), PÉREZ (A.) - Une limitation centuriée à Nîmes et à Orange. In: BURNOUF (J.), BRAVARD (J.-P.), CHOUQUER (G.) éd. - La dynamique des paysages protohistoriques, antiques, médiévaux et modernes. Actes des XVII rencontres d'archéologie et d'histoire d'Antibes, 19-20-21 octobre 1996. Sophia Antipolis, éd. APDCA, 1997, pp. 69-84.

Barberan et al. 2002 : BARBERAN (S.), FABRE (L.), MAUFRAS (O.), PETITOT (H.), POMAREDES (H.), SAUVAGE (L.), THERNOT (R.) - Les villae de La Ramière à Roquemaure, Gard. In : Archéologie du TGV Méditerranée, Fiches de Synthèse. Tome 3, Antiquité, Moyen Âge, Époque moderne. Lattes, 2002, pp. 889-919 (Monographies d'Archéologie Méditerranéenne, 10).

Bazzana et al. 1987 : BAZZANA (A.), BERTRAND (M.), CRESSIER (P.), GUICHARD (P.), MONTMESSIN (Y.) - L'hydraulique agraire dans l'Espagne médiévale. In : DE REPARAZ (A.) - L'eau et les hommes en Méditerranée. Actes du colloque du G.I.S. Paris, CNRS, 1987, pp. 43-66.

Bellamy, Hitchner 2000 : BELLAMY (), HITCHNER () - Les fouilles de La Mérindole (1991-1993) : un bâtiment annexe à la villa et un cimetière du haut Moyen Âge. In : LEVEAU (Ph.), SAQUET (J.-P.) éd. - Milieu et sociétés dans la vallée des Baux. Actes du colloque de Mouriès. Montpellier, 2000, 213-230 (Suppl. 31 à la RAN).

Billiard 1913 : BILLIARD (R.) - La vigne dans l'Antiquité. Lyon, H. Lardanchet, 1913, $560 \mathrm{p}$.

Boissinot 1995 : BOISSINOT (Ph.) - L'empreinte des paysages hellénistiques dans les formations holocènes de Saint-Jean-du-Désert (Marseille). Méditerranée, 3-4, 1995, pp. 33-40.

Boissinot 2001 : BOISSINOT (Ph.) - Archéologie des vignobles antiques du sud de la Gaule. In: BRUN (J.-P.), LAUBENHEIMER (F.) dir. - La viticulture en Gaule. Gallia, 58, 2001, pp. 45-68.

Brun 1986 : BRUN (J.-P.) - L'oléiculture antique en Provence: les huileries du département $d u$ Var. Montpellier, 1986 (suppl. à la RANarb, 15).

Brun, Congès 1988 : BRUN (J.-P.), CONGÈS (G.) - Autour d'Heraclea Caccabaria. Archéologie de la côte des Maures. Toulon, CAV, 1988.

Buffat 1994 : BUFFAT (L.) - L'occupation humaine de la commune de Tresques (Gard) durant l'Antiquité tardive et le haut Moyen Âge. Mémoire de Maîtrise, Université Montpellier III, 1994, 70 p. + fig.

Buffat 1996 : BUFFAT (L.) - Premières observations sur la villa antique et médiévale de Mayran à Saint-Victor-la-Coste (Gard). Mémoire de DEA, Université Montpellier III, 1996, 73 p. + fig.

Buffat 2004 : BUFFAT (L.) - L'économie domaniale en Gaule Narbonnaise: les villae de la cité de Nîmes. Thèse de doctorat Nouveau Régime, Aix-en-Provence, 2004, 456 p.
Buffat, Petitot 1996 : BUFFAT (L.), PETITOT (H.) avec la coll. de LAROZAS (Chr.) - Habitat gallo-romain et aménagements périphériques: l'exemple d'Aubarnes à Tresques. Archéologie en Languedoc, 20-2, 1996, pp. 89-97.

Buffat, Petitot 1998 : BUFFAT (L.), PETITOT (H.) - Une activité métallurgique tardo-antique sur l'établissement de Mayran (SaintVictor-la-Coste, Gard). In : FEUGÈRE (M.), SERNEELS (V.) dir. Recherches sur l'économie du fer en Méditerranée nord-occidentale. lieu d'édition. Montagnac, 1998, pp. 175-180 (Instrumentum, 4).

Buffat, Pellecuer 2001 : BUFFAT (L.), PELLECUER (Chr.) avec la coll. de MAUNÉ (S.), POMAREDES (H.) - La viticulture antique en Languedoc-Roussillon. In: BRUN (J.-P.), LAUBENHEIMER (F.) dir. - La viticulture en Gaule, Gallia , 58, 2001, pp. 91-111.

Buffat et al. 2002 : BUFFAT (L.), CHRISTOL (M.), PELAQUIER (E.), PETITOT (H.) - Le problème d'interprétation des établissements ruraux de grande dimension: quelques cas en Languedoc. RANarb, 35, 2002, pp. 199-239.

CAG 30/2: PROVOST (M.) dir. - Carte archéologique de la Gaule, Le Gard. 30/2. Paris, MSH, 1999, 399 p.

CAG 30/3: PROVOST (M.) dir. - Carte archéologique de la Gaule, Le Gard. 30/3. Paris, MSH, 1999, 465 p.

CAG 37: PROVOST (M.) dir. - Carte archéologique de la Gaule, L'Indre-et-Loire. 37. Paris, MSH, 1988.

CAG 83/1 : BRUN (J.-P.) avec la collab. de BORRÉANI (M.) - Carte archéologique de la Gaule, Le Var. 83/1. Paris, MSH, 1999.

CAG 83/2 : BRUN (J.-P.) avec la collab. de BORRÉANI (M.) - Carte archéologique de la Gaule, Le Var. 83/2. Paris, MSH, 1999.

Cathma 1993 : CATHMA - LEENHARDT (M.), RAYNAUD (Cl.), SCHNEIDER (L.) dir. - Céramiques languedociennes du haut Moyen Âge (VII ${ }^{\mathrm{e}}-\mathrm{XI}^{\mathrm{e}} \mathrm{s}$.), études micro-régionales et essai de synthèse. Archéologie du Midi Médiéval, 11, 1993, pp. 111-228.

Chouquer 1994 : CHOUQUER (G.) - Cadastre d'Orange et archéologie : résultats et hypothèses. In : FAVORY (F.), FICHES (J.-L.) dir. - Les campagnes de la France Méditerranéenne dans l'Antiquité et le Haut Moyen Âge: approches micro-régionales. Paris, 1994, pp. 51-55 (Documents d'Archéologie Française, 42).

Clément 1989 : CLÉMENT (P.-A.) - Églises romanes oubliées du Bas-Languedoc. Montpellier, Presses du Languedoc, 1989.

Columelle (I à VI): Columelle - NISARD (M.) - De l'Agriculture. In: Les Agronomes latins, Caton, Varron, Columelle, Palladius. Paris, Librairies Garnier Frères, 1851.

Dechandol et al. 1983 : DECHANDOL (H.), FEUILLET (M. P.), ODIOT (Th.) - Le grand domaine viticole du Mollard. Histoire et archéologie, 78, 1983.

Esteban et al. 2000 : ESTEBAN (A.), BUFFAT (L.), GUERRE (J.), PETITOT (H.) - Une mosaïque du $\mathrm{I}^{\text {er }} \mathrm{s}$. ap. J.-C. découverte à Saint-Paul-les-Fonts - Sondages de repérages sur une villa galloromaine implantée en bordure du couloir rhodanien. Rhodanie, 76, 2000, pp. 25-40.

Fabre t t al. 1999 : FABRE (G.), FICHES (J.-L.), PAILLET (J.-L.) L'aqueduc de Nîmes et le drainage de Clausonne: hypothèses sur le financement de l'ouvrage et sur l'identité de son concepteur. In: BEDON (R.) éd. - Les aqueducs de la Gaule romaine et des régions voisines. Caesarodunum, XXXI, 1999, pp. 193-219.

Faraud 1952 : FARAUD (M.) - Vestiges romains à Saint-Victor. $D u$ Castellas à Meyran. Rapport déposé au SRA Montpellier, 1952, 4 p.

Faraud 1972 : FARAUD (L.) - Rapport de fouille de sauvetage d'une sépulture à inhumation avec mobilier. Ermitage de Mayran. Rapport déposé au SRA Montpellier, 1972, 4 p. 
Favory 1997 : FAVORY (F.) - Retour critique sur les centuriations du Languedoc oriental, leur existence et leur datation. In : CHOUQUER ((G.) dir. - L'analyse des systèmes spatiaux. Les formes $d u$ paysage. T. 3. Paris, 1997, 96-126.

Favory et al. 1993 : FAVORY (F.), MALVIS (J.-M.), MERCIER (C.), RAYNAUD (Cl.), ROGER (K.) - Limitations antiques et morphologie parcellaire dans le Lunellois (Hérault). RANarb, 26, 1993, pp. 139-170.

FORULA 1980 : Foyer Rural de Lavérune - Lavérune - Rapport de fouilles. Rapport déposé au SRA Montpellier, 1980.

Gateau 1993 : GATEAU (F.) - Gignac-la-Nerthe, La Pousaraque. In : PELLECUER (Chr.) dir. - Formes de l'habitat rural en Gaule Narbonnaise, 1. Juan-les-Pins, APDCA, 1993.

Gilles 1990 : GILLES (K.-J.) - Die neu entdeckte spätrömische Weinkelter von Brauneberg. Funde und Ausgrabungen im Bezirk Trier, 22, 1990, pp. 33-45.

Gilles 1991 : GILLES (K.-J.) - Eine weitere römische Weinkelter aus Brauneberg. Funde und Ausgrabungen im Bezirk Trier, 23, 1991, pp. 20-32.

Goury, Leclaire 1992 : GOURY (D.), LECLAIRE (A.) - La villa gallo-romaine de Mayran à Saint-Victor-la-Coste. Rhodanie, 41, 1992, pp. 15-24.

Laubenheimer 1990 : LAUBENHEIMER (F.) - Sallèles-d'Aude - Un complexe de potiers gallo-romain. Paris, MSH, 1990, $160 \mathrm{p}$. (Documents d'Archéologie Française, 26).

Leveau 1984 : LEVEAU (Ph.) - Caesarea de Maurétanie, une ville romaine et ses campagnes. Rome, 1984 (Collection de l'École Française de Rome, 70).

Maufras, Fabre 1998 : MAUFRAS (O.), FABRE (L.) - Une forge tardive (fin $I V^{e}-V^{e} s$.) sur le site de la Ramière (Roquemaure, Gard). In : FEUGÈRE (M.), SERNEELS (V.) dir. - Recherches sur l'économie du fer en Méditerranée nord-occidentale. Montagnac, 1998, pp. 210-221 (Monographies Instrumentum, 4).

Mauné 1998 : MAUNÉ (S.) - Les campagnes de la cité de Béziers dans l'Antiquité (partie nord-orientale) (II ${ }^{e}$ s. av. J.-C.-VI S.ap. $J$.-C.). Montagnac, M. Mergoil, 1998.

Mauné, Paillet 2003 : MAUNÉ (S.), PAILLET (J.-L.) - Stockage et transformation des céréales dans l'économie rurale de Gaule Narbonnaise ( $\mathrm{I}^{\mathrm{er}}-\mathrm{II}^{\mathrm{e}}$ siècle ap. J.-C.). L'exemple des moulins hydrauliques de Vareilles et de L'Auribelle-Basse (Hérault). In: ANDERSON (P.C.), CUMMINGS (L.S.), SCHIPPERS (T.K.), SIMONEL (B.) - Le traitement des récoltes: un regard sur la diversité, du Néolithique au présent. Actes des rencontres d'Antibes (17-19 octobre 2002). Antibes, éd. APDCA, 2003, pp. 295-326.

Monteil et al. 1999 : MONTEIL (M.), BARBERAN (S.), PISKORZ (M.), VIDAL (L.) - Culture de la vigne et traces de plantation des $\mathrm{II}^{\mathrm{e}}$ et $\mathrm{I}^{\mathrm{er}}$ siècles avant J.-C. dans la proche campagne de Nîmes (Gard, France). RANarb, 32, 1999, pp. 67-123.
Nìn 1987 : NIN (N.) - La voie aurélienne et ses abords à Aix-enProvence. Nouvelles données sur un paysage périurbain. RANarb, 20, 1987, pp. 191-280.

Olive 1993 : OLIVE (Chr.) - Péret, Combe de Fignols. In: PELLECUER (Chr.) dir. - Formes de l'habitat rural en Gaule Narbonnaise, 1. APDCA, Juan-les-Pins, 1993

Palladius : MARTIN (R.) éd. - Palladius - Traité d'agriculture - Livres I-II. Paris, Les Belles Lettres, 1976, 209 p.

Pellecuer 2000 : PELLECUER (Chr.) - La villa des Près-Bas (Loupian, Hérault) dans son environnement. Contribution à l'étude des villae et de l'économie domaniale en Narbonnaise. Thèse de Doctorat nouveau régime, Université Aix-Marseille I, 2000, 2 vol., $565 \mathrm{p}$.

Petitot, Vidal 1993 : PETITOT (H.), VIDAL (L.) - Techniques agricoles et construction des paysages centuriés de la campagne nîmoise. Dialogues d'Histoire Ancienne, 1993, pp. 310-313.

Pelletier, Poguet 2000 : PELLETIER (J.-P.), POGUET (M.) Eyguières: 1'occupation du site Saint-Pierre 1 de l'âge du Fer au $\mathrm{X}^{\mathrm{e}}$ siècle et premières études des céramiques. In: LEVEAU $(\mathrm{Ph}$.$) ,$ SAQUET (J.-P.) - Milieu et sociétés dans la vallée des Baux. Actes du colloque de Mouriès, Montpellier, 2000, pp. 275-338 (suppl. à la RANarb, 31).

Py 1993 : PY (M.) dir. - DICOCER. Dictionnaire des céramiques antiques en Méditerranée nord-occidentale. Lattes, 1993, 624 p. (Lattara, 6).

Raynaud 1990 : RAYNAUD (Cl.) - Le village gallo-romain et médiéval de Lunel Viel (Hérault). Paris, Les Belles Lettres, 1990, 353 p.

Sigaut 1978 : SIGAUT (F.) - Les réserves de grains à long terme. Lille, MSH, 1978.

Varron, I: Varron - HEURGON (J.) éd - Économie rurale - Livre I. Paris, Les Belles Lettres, 1978, 189 p.

Vidal 1998 : VIDAL (L.) - Un domaine antique en Biterrois: La Domergue à Sauvian (Hérault). In: CLAVEL-LÉVÊQUE (M.), VIGNOT (A.) éd. - Cité et Territoire II. Actes du colloque européen de Béziers (24-26 octobre 1997). Paris, Les Belles Lettres, 1998, pp. 31-53.

Vidal 2000 : VIDAL (L.) - Mise en valeur et aménagement des campagnes de la Protohistoire au Moyen Âge dans la France du Sud. Thèse de Doctorat nouveau régime, Université Montpellier III, 3 vol., 513 p. + fig.

Vidal, Petitot 2003 : VIDAL (L.), PETITOT (H.) - Pour une archéologie de la limite et du bornage; données antiques de la Gaule Narbonnaise. In: Actualité de la Recherche en Histoire et Archéologies agraires. Actes du colloque international AGER V (Besançon septembre 2000). Besançon, Presses Universitaires Franc-Comtoises, 2003, pp. 79-96. 


\section{ANNEXE 1: INVENTAIRE DU MOBILIER DE PROSPECTION}

\section{Zone Bas-Empire, Haut Moyen Âge, Moyen Âge central}

Parcelle AH 73 (cumul de 16 tests de $100 \mathrm{~m}^{2}$ )

Sigillée sud-gauloise : 7 fr. (Drag. 27), claire-B/luisante: $23 \mathrm{fr}$. (Portout 37a [2]), claire D: 5 fr. (Hayes 67), claire africaine indét.: 1 fr., claire engobée oxydante: 4 fr. (CL-ENG B11), fine indéterminée : 2 fr., céramique non tournée : $18 \mathrm{fr}$. (CNTLOR U7, décor cordon, décor peigné), claire récente: $11 \mathrm{fr}$. (CL-REC $13 \mathrm{~g}$ ), oxydante micacée: $5 \mathrm{fr}$, pisolithes oxydante : 22 fr. (PISO A1, B5a, B5c [3], C2b), pisolithes réductrice: 3 fr., kaolintique réductrice: 267 fr. (KAOL A12, A29a [2], A29b, B24, CATHMA 2a, 5a, 5b, 5c [2], 5d, indét.), molette [5], cannelures [8]), sableuse réductrice: 2 fr., kaolintique oxydante: 18 fr. (A18, B24a), vernissée de l'Uzège : 6 fr., vernissée moderne: 2 fr., amphore gauloise: $9 \mathrm{fr}$. (G4), amphore gauloise sableuse: 3 fr. (G1 anse), amphore de Bétique: $7 \mathrm{fr}$. (panse Dr. 20 [1], panse Dr. 23 [5], panse Ha. 70 [1]), amphore africaine: 24 fr. (Keay 25), amphore indéterminée: 5 fr., amphore hispanique: $1 \mathrm{fr}$, amphore italique: $1 \mathrm{fr}$,, amphore massaliète impériale: $1 \mathrm{fr}$.

Tubulure calcaire: 2 fr., tubulure orange micacée: $1 \mathrm{fr}$, brique d'hypocauste (pilette): $1 \mathrm{fr}$., scories de fer: $37 \mathrm{fr}$., verre bleuté : 1 anse, verre moderne: $17 \mathrm{fr}$.

Parcelle AH 123 (1 test)

Kaolinitique réductrice: 13 fr. (Cathma 5b), pisolithes oxydante : 2 fr. (PISO C2c), pisolithes réductrice: 1 fr., non tour- née: 2 fr., amphore de Bétique: 1 (panse Dr. 23), amphore africaine: $3 \mathrm{fr}$.

Silex : 2 fr.

Parcelle AH 124 (1 test)

Sigillée sud-gauloise: 1 fr. (Drag. 33), claire récente: 1 fr., oxydante micacée: 1 fr., kaolinitique oxydante: 1 fr., kaolinitique réductrice: 15 fr. (KAOL B24a), pisolithes oxydante: 2 fr. (PISO C2c), non tournée: 4 fr., vernissée de 1'Uzège: 3 fr., amphore gauloise: 1 fr., amphore gauloise sableuse: 1 fr., amphore hispanique: $1 \mathrm{fr}$.

\section{Zone Haut-Empire}

Parcelles AI 152, 191, 195, 198 (cumul de 4 tests de $100 \mathrm{~m}^{2}$ ) Sigillée sud-gauloise: 11 fr. (Drag. 35/36 [2], 44a), claire B: 1 fr., claire récente: 9 fr. (CL-REC 19b), oxydante micacée: 6 fr. (COM-O-M A4), kaolinitique oxydante: 15 fr., kaolinitique réductrice: 17 fr. (KAOL B8a, CATHMA 3, 5b), sableuse oxydante: 1 fr., sableuse réductrice: 3 fr., vernissée de l'Uzège: $5 \mathrm{fr}$., amphore gauloise: $11 \mathrm{fr}$, amphore gauloise sableuse: 16 fr. (G1 [2]), amphore gauloise (pâte mixte) : 7 fr., amphore de Bétique : 5 fr. (Dr. 7/11, panse Dr. 7/11, panse Dr. $20[2])$

Tubulure indifférenciée: 4 fr., tubulure orange micacée: 1 fr., tubulure calcaire: 1 fr., tesselle en pierre noire: 1 fr., meule en basalte: 1 fr., scories de fer: 2 fr.

\section{ANNEXE 2: INVENTAIRE DES CÉRAMIQUES DU HAUT MOYEN ÂGE ET DU MOYEN ÂGE CENTRAL}

\begin{tabular}{|c|c|c|c|c|c|c|c|c|c|}
\hline US cér. & clair-b & com-o-m & Kaol-o & Kaol-r & piso-o & piso-r & a-hisp & a-indét & Total \\
\hline US 1150 & 1 & 3 & 2 & 61 & 2 & 1 & 2 & 2 & $\mathbf{7 4}$ \\
\hline US 1250 & & & & 6 & & & & & $\mathbf{6}$ \\
\hline US 1280 & & & & 5 & 1 & & & & $\mathbf{6}$ \\
\hline
\end{tabular}

Tabl. 1. Inventaire des céramiques de la phase D1.

\begin{tabular}{|c|c|c|c|c|c|c|c|c|c|c|c|c|c|c|c|}
\hline \multirow{2}{*}{ US cér. } & \multirow{2}{*}{$\begin{array}{l}\text { NFR } \\
\text { Kaol } \\
\end{array}$} & \multicolumn{11}{|c|}{ Nbd Cathma } & \multicolumn{2}{|c|}{ Décor } & \multirow{2}{*}{$\begin{array}{c}\text { NFR } \\
\text { gallo-romains }\end{array}$} \\
\hline & & $2 \mathrm{a}$ & $5 b$ & $5 \mathrm{c}$ & $6 a$ & $6 \mathrm{~b}$ & $6 c$ & $7 a$ & $7 b$ & 9 & 12 & indét. & can. & poinç & \\
\hline US 1102 & 15 & & & & & & & & 1 & & & 1 & & & 3 \\
\hline US 1114 & 5 & 1 & & & & & & & & & & & & & \\
\hline US 1134 & 17 & 1 & & & & & & & 1 & & & 1 & & & \\
\hline US 1288 & 59 & & 2 & & & & 2 & 1 & 1 & 1 & 1 & 1 & 4 & & 3 \\
\hline US 1290 & 36 & & & 1 & & 1 & 1 & & & & & 1 & 1 & & 4 \\
\hline US 1292 & 51 & & & & 1 & & & & 1 & & & & 3 & 1 & 9 \\
\hline US 1363 & 7 & & & & 1 & 1 & & & & & & & & & 3 \\
\hline US 1364 & 2 & & & & & & & & & & & & 1 & & 2 \\
\hline
\end{tabular}

Tabl. 2. Inventaire des céramiques de la phase D2. 


\begin{tabular}{|c|c|c|c|c|c|c|c|c|c|c|c|c|c|c|c|c|c|c|c|c|c|c|}
\hline \multirow{2}{*}{ US cér. } & \multirow{2}{*}{$\begin{array}{l}\text { NFR } \\
\text { Kaol }\end{array}$} & \multicolumn{14}{|c|}{ Nbd Cathma } & \multicolumn{6}{|c|}{ Décor } & \multirow{2}{*}{\begin{tabular}{|c|} 
NFR résiduels \\
gallo-romains
\end{tabular}} \\
\hline & & $1 \mathrm{a}$ & 3 & $5 \mathrm{a}$ & $5 b$ & $5 c$ & $5 \mathrm{~d}$ & $6 \mathrm{a}$ & $6 c$ & $6 \mathrm{~d}$ & $7 a$ & $7 b$ & 9 & $10 \mathrm{~b}$ & indét. & can. & molette & ondé & polis & poinç. & crois. & \\
\hline US 1002 & 10 & & & & & & & & 1 & & & & & & & 1 & 1 & & & & & 1 \\
\hline US 1011 & 32 & & & & 1 & & 1 & & & & & & & & 1 & 13 & & & & & & \\
\hline US 1017 & 24 & & & & & & & & & & & & & & & 6 & 1 & & & & & \\
\hline US 1057 & 90 & & & 1 & & & 1 & & & & & 1 & & & & 32 & & & & 4 & & 2 \\
\hline US 1058 & 41 & & & & & 1 & 1 & 1 & & & & 1 & & & & 4 & 4 & & 1 & & & 2 \\
\hline US 1063 & 73 & & 1 & & & & 1 & & & & & & & & & 10 & 8 & & & & 1 & 3 \\
\hline US 1084 & 20 & & & & 1 & & & & & & & & & & & 3 & 2 & & & & & \\
\hline US 1085 & 15 & & & 1 & & & & & & & & & & & & 1 & & & & & & 5 \\
\hline US 1088 & 10 & & & & 1 & & & & & & & & & & & & & & & & & 1 \\
\hline US 1099 & 81 & & & & & & 1 & & & & 1 & 1 & & & 3 & 31 & 3 & & & & & 7 \\
\hline US 1101 & 17 & & & & & & & & & & & & & & & 2 & 1 & & & & & \\
\hline US 1111 & 16 & & & & & & 1 & & & & & & & & & 3 & 1 & & & & & 1 \\
\hline US 1113 & 82 & & & & 3 & 1 & 1 & & & & & & & & & 3 & 1 & & & & & 2 \\
\hline US 1119 & 86 & & & & & & 3 & & & 1 & & & & & 1 & 6 & 1 & & & & & 3 \\
\hline US 1120 & 10 & & & & & & & & & & & & & & 1 & 1 & & & & & & 1 \\
\hline US 1244 & 111 & 1 & & & & & 1 & & & & & & 1 & 1 & 2 & 9 & 3 & 1 & & 1 & & 5 \\
\hline US 1246 & 5 & & & & 1 & & & & & & & & & & 1 & & & & & & & \\
\hline US 1248 & 101 & & & & 1 & & & & & & & 1 & & & & 49 & & & & & & 5 \\
\hline US 1253 & 15 & & & & 1 & & & & & & & & & & & 4 & & & & & & 2 \\
\hline US 1258 & 16 & & & & & & 1 & & & & & & & & & 6 & 3 & & & & & \\
\hline US 1269 & 11 & & & & & & & & & & & & & & & 1 & & 1 & & & & \\
\hline US 1282 & 7 & & & & & & & & & & & 1 & & & & 4 & & & & & & 2 \\
\hline US 1355 & 10 & & & & & & & & & & & & & & & 2 & & & & & & \\
\hline US 1357 & 9 & & & & & & & & & & & & & & & & & & 1 & & & 2 \\
\hline US 1412 & 6 & & & & & & & & & & & & & & & 1 & & & & 1 & & 1 \\
\hline
\end{tabular}

Tabl. 3. Inventaire des céramiques de la phase D3.

\begin{tabular}{|c|c|c|c|c|c|c|c|c|c|c|c|c|c|c|c|c|c|}
\hline \multirow{2}{*}{ US Cér. } & \multirow{2}{*}{$\begin{array}{l}\text { NFR } \\
\text { Kaol }\end{array}$} & \multicolumn{9}{|c|}{ Nbd Cathma } & \multicolumn{6}{|c|}{ Décor } & \multirow{2}{*}{$\begin{array}{l}\text { NFR résiduels } \\
\text { gallo-romains }\end{array}$} \\
\hline & & $2 b$ & 3 & $5 a$ & $5 b$ & $5 c$ & $5 \mathrm{~d}$ & $6 \mathrm{~b}$ & & indét. & can. & molette & ondé & polis & poinç & crois. & \\
\hline US 1086 & 23 & & & & & & & & & & 3 & 2 & & & & & 6 \\
\hline US 1087 & 27 & & & & & & & & & 1 & & & & & 1 & & 1 \\
\hline US 1103 & 109 & & & 1 & 1 & 1 & 1 & & & & 3 & 14 & & & & & 2 \\
\hline US 1115 & 10 & & & & 1 & & & & & & & 2 & & & & & \\
\hline US 1152 & 35 & & & & 1 & & & & & 1 & & & & & & & 3 \\
\hline US 1158 & 66 & & & & 1 & & 1 & & & & & 2 & & 1 & & & 3 \\
\hline US 1160 & 11 & & & & & & & & & & & 3 & & & & & 2 \\
\hline US 1164 & 35 & & & 1 & & & & & & 1 & 2 & 1 & & & & & 1 \\
\hline US 1166 & 14 & & & & & & & & & & & 1 & & & & & \\
\hline US 1168 & 7 & & & 1 & & 1 & & & & & & & & & & & \\
\hline US 1170 & 33 & & & & & & & & & & & 1 & 1 & & & & \\
\hline US 1171 & 30 & & & 2 & & & & & & & & 1 & & 1 & & & 1 \\
\hline US 1172 & 62 & & & & & & & & & 1 & & 1 & & & & & \\
\hline US 1190 & 10 & & & & & & & & 1 & 1 & & 1 & & & & & 1 \\
\hline US 1196 & 18 & & & & & & & & & & & 2 & & & 1 & & 7 \\
\hline US 1198 & 21 & & & & 1 & & & 1 & & & & & & & & 1 & 4 \\
\hline US 1202 & 21 & & & 1 & & & & & & & & & & & & 2 & 3 \\
\hline US 1205 & 8 & & & & & & & & & & & 1 & & & & & 5 \\
\hline US 1206 & 20 & & & & & & 1 & & & & 2 & 1 & & & & & 3 \\
\hline US 1216 & 28 & & & & & & & & & & & 1 & & & & 1 & 6 \\
\hline US 1217 & 24 & & & & & & & & & 2 & 2 & & & & & & 5 \\
\hline US 1269 & 11 & & & & & & & & & & 1 & & 1 & & & & \\
\hline US 1273 & 8 & & & & & & & & & & & 1 & & & & & \\
\hline US 1274 & 7 & & 1 & & & & & & & & & 1 & & & & & 1 \\
\hline US 1278 & 8 & & & & & & & & & & & 3 & & & & & 2 \\
\hline US 1284 & 18 & & & & 1 & & & & & & & 3 & & & & & 1 \\
\hline US 1296 & 12 & & & & & & & & & & & & 1 & & & & \\
\hline US 1350 & 4 & & & & & & & & & & & & & & & & \\
\hline US 1352 & 7 & & & & & & & & & & 1 & 1 & & & & & \\
\hline US 1353 & 31 & 1 & 1 & 1 & 1 & & & & & & 3 & 3 & & & & & 4 \\
\hline US 1355 & 10 & & & & & & & & & & 2 & & & & & & \\
\hline US 1357 & 9 & & & & & & & & & & & & & 1 & & & 2 \\
\hline
\end{tabular}

Tabl. 4. Inventaire des céramiques de la phase D4. 


\begin{tabular}{|c|c|c|c|c|c|c|c|c|c|c|c|c|c|c|c|c|c|c|c|}
\hline \multirow{2}{*}{ US Cér. } & \multirow{2}{*}{$\begin{array}{l}\text { NFR } \\
\text { Kaol }\end{array}$} & \multicolumn{12}{|c|}{ Nbd Cathma } & \multicolumn{5}{|c|}{ Décor } & \multirow{2}{*}{$\begin{array}{l}\text { NFR résiduels } \\
\text { gallo-romains }\end{array}$} \\
\hline & & $1 \mathrm{a}$ & $1 \mathrm{~b}$ & $2 b$ & 3 & $5 a$ & $5 b$ & $5 c$ & $5 \mathrm{~d}$ & $6 \mathrm{~b}$ & $7 \mathrm{a}$ & $7 \mathrm{~b}$ & indét. & can. & molette & polis & poin & crois. & \\
\hline US 1154 & 67 & & & & 2 & & & 1 & & & & & 1 & & 5 & & 1 & & 4 \\
\hline US 1157 & 5 & & & & 1 & & & & & & & & 1 & & & & & & \\
\hline US 1160 & 11 & & & & & & & & & & & & & & 3 & & & & 2 \\
\hline US 1166 & 14 & & & & & & & & & & & & & & 1 & & & & \\
\hline US 1175 & 22 & & & & & & & & & & & & & & 2 & & & & \\
\hline US 1176 & 30 & & & & 1 & & 1 & 1 & & & & & 1 & & 2 & & & & 1 \\
\hline US 1178 & 107 & & & & 1 & & & 1 & & & & & & 1 & 3 & & & & 16 \\
\hline US 1180 & 123 & & & & 2 & & & & 1 & & & & & 3 & 3 & 1 & & 1 & 2 \\
\hline US 1182 & 86 & & & & 2 & & & & & & & & 1 & & & & & & 2 \\
\hline US 1184 & 114 & & & & 3 & 3 & & 3 & & & & & & & 5 & & 1 & & 5 \\
\hline US 1190 & 10 & & & & & & & & & & & 1 & 1 & & 1 & & & & 1 \\
\hline US 1194 & 27 & & & & 1 & & & & & & & & & & & & & & 1 \\
\hline US 1196 & 18 & & & & & & & & & & 1 & & & & 2 & & 1 & & 7 \\
\hline US 1198 & 21 & & & & & & 1 & & & 1 & & & & & & & & 1 & 4 \\
\hline US 1204 & 30 & & & & 1 & & & & & & & & & & 3 & 2 & & & 4 \\
\hline US 1205 & 8 & & & & & & & & & & & & & & 1 & 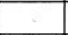 & & & 5 \\
\hline US 1216 & 28 & & & & & & & & & & & & & & 1 & & & 1 & 6 \\
\hline US 1240 & 110 & 1 & & & 1 & & & 1 & 1 & & & & & 2 & 2 & & & & 6 \\
\hline US 1256 & 125 & & 1 & & 1 & 1 & & 1 & & & & & 1 & 1 & 5 & & 1 & & 5 \\
\hline US 1273 & 8 & & & & & & & & & & & & & & 1 & & & & \\
\hline US 1274 & 7 & & & & 1 & & & & & & & & & & 1 & & & & 1 \\
\hline US 1278 & 8 & & & & & & & & & & & & & & 3 & & & & 2 \\
\hline US 1350 & 4 & & & & & & & & & & & & & & & & & & \\
\hline US 1353 & 31 & & & 1 & 1 & 1 & 1 & & & & & & & 3 & 3 & & & & 4 \\
\hline US 1372 & 38 & & & & 2 & & 1 & & & & & & & & & & & & 1 \\
\hline
\end{tabular}

Tabl. 5. Inventaire des céramiques de la phase D5. 